Supporting Information for:

\title{
Lewis acid effects on calculated ligand electronic parameters
}

James P. Shanahan and Nathaniel K. Szymczak*

Department of Chemistry, University of Michigan, Ann Arbor, Michigan 48109, United States 


\section{Table of Contents:}

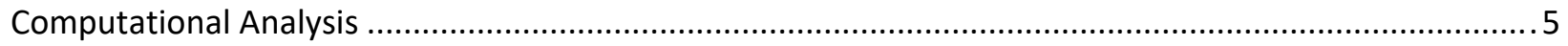

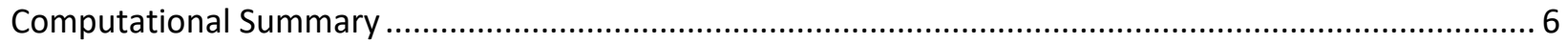

Figure S1. Table of calibrated $\Delta \mathrm{v}_{\mathrm{CO}(\mathrm{a} 1)}\left(\mathrm{cm}^{-1}\right)$ for the series of ligands and Lewis acid adducts. .................. 6

Figure S2. Table of $\Delta \mathrm{G}(\mathrm{kcal} / \mathrm{mol})$ for the series of ligands and Lewis acid adducts................................ 7

Figure S3. Table of $\Delta \mathrm{H}(\mathrm{kcal} / \mathrm{mol})$ for the series of ligands and Lewis acid adducts................................ 8

Figure S4. Table of calibrated $\mathrm{v}_{\mathrm{CO}(\mathrm{a})}\left(\mathrm{cm}^{-1}\right)$ for the series of ligands and Lewis acid adducts..................... 9

Figure S5. Representative rendered structures of $\mathrm{BF}_{3}$ adducts of $\left[\mathrm{Ni}(\mathrm{CO})_{3}-\mathrm{L}\right]^{\mathrm{n}}$ complexes of $(\mathrm{A}) \mathrm{X}$-type ligands, (B) N-heterocycle ligands, and (C) small molecules............................................................... 10

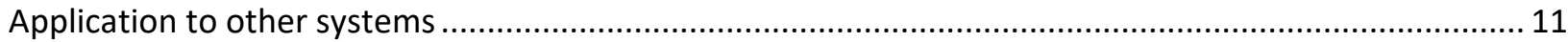

Figure S6. Lewis acid behavior of $\mathrm{Fe}(\mathrm{X})_{2}\left({ }^{\mathrm{BBN}} \mathrm{PDP}^{\mathrm{tBu}}\right)$ complexes $^{10}$ and parameters from $\left[\mathrm{Ni}(\mathrm{CO})_{3} \mathrm{X}\right]^{-}$analysis

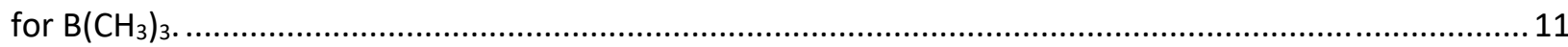

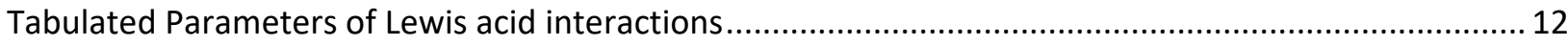

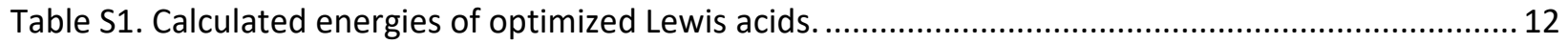

Table S2. Calculated energies for Fluoride Ion Affinity Lewis acid scale. ............................................ 13

Table S3. Calculated energies for hydride ion affinity. ....................................................................... 13

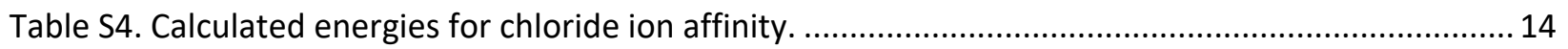

Table S5. Calculated energies for bromide ion affinity ........................................................................ 14

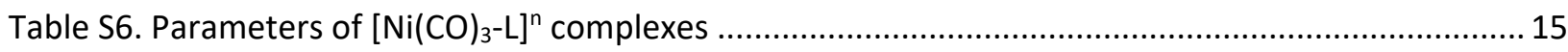

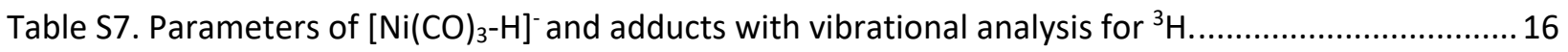

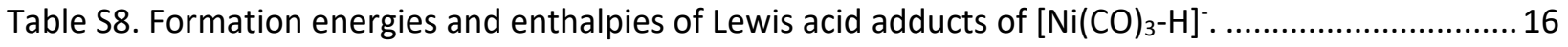

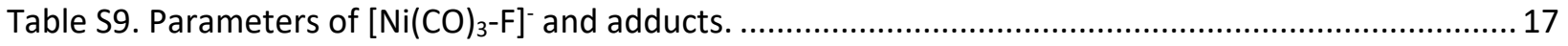

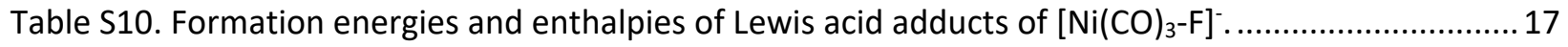

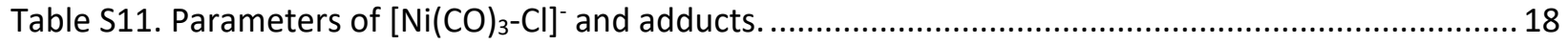

Table S12. Formation energies and enthalpies of Lewis acid adducts of $\left[\mathrm{Ni}(\mathrm{CO})_{3}-\mathrm{Cl}\right]^{-} \ldots \ldots \ldots \ldots \ldots \ldots \ldots \ldots \ldots . . . . . . . . . . . .18$

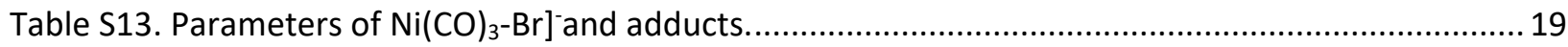

Table S14. Formation energies and enthalpies of Lewis acid adducts of $\left[\mathrm{Ni}(\mathrm{CO})_{3}-\mathrm{Br}\right]^{-} \ldots \ldots \ldots \ldots \ldots \ldots \ldots \ldots \ldots . . . . . . . . . . .19$

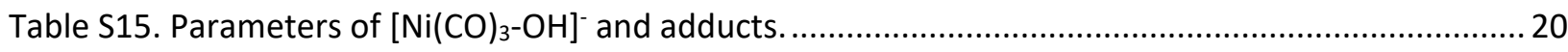

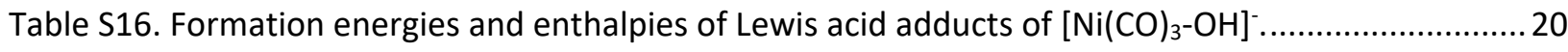

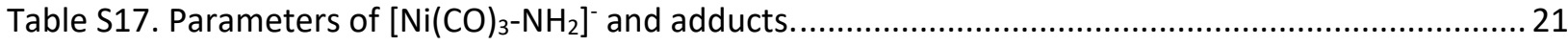

Table S18. Formation energies and enthalpies of Lewis acid adducts of $\left[\mathrm{Ni}(\mathrm{CO})_{3}-\mathrm{NH}_{2}\right]^{-}$...................... 21

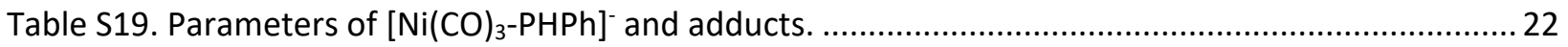


Table S20. Formation energies and enthalpies of Lewis acid adducts of $\left[\mathrm{Ni}(\mathrm{CO})_{3}-\mathrm{PHPh}\right]^{-}$. .22

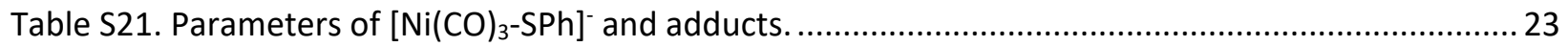

Table S22. Formation energies and enthalpies of Lewis acid adducts of $\left[\mathrm{Ni}(\mathrm{CO})_{3}-\mathrm{SPh}\right]^{-} \ldots \ldots \ldots \ldots \ldots \ldots \ldots \ldots . . . . . . . . . . .23$

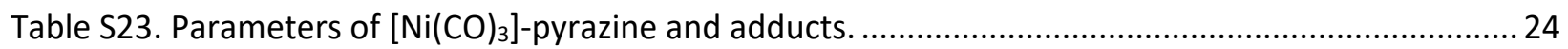

Table S24. Formation energies and enthalpies of Lewis acid adducts of $\left[\mathrm{Ni}(\mathrm{CO})_{3}\right]$-pyrazine....................24

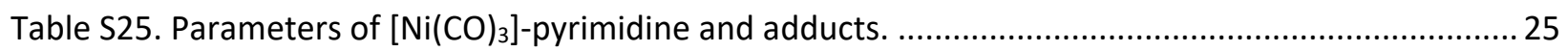

Table S26. Formation energies and enthalpies of Lewis acid adducts of $\left[\mathrm{Ni}(\mathrm{CO})_{3}\right]$-pyrimidine. ...............2 25

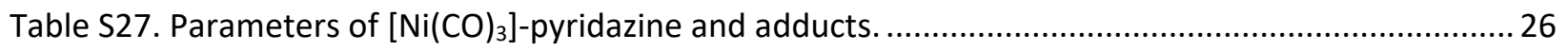

Table S28. Formation energies and enthalpies of Lewis acid adducts of $\left[\mathrm{Ni}(\mathrm{CO})_{3}\right]$-pyridazine................26

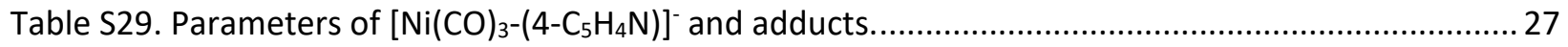

Table S30. Formation energies and enthalpies of Lewis acid adducts of $\left[\mathrm{Ni}(\mathrm{CO})_{3}-\left(4-\mathrm{C}_{5} \mathrm{H}_{4} \mathrm{~N}\right)\right]^{-} \ldots \ldots \ldots \ldots \ldots . .27$

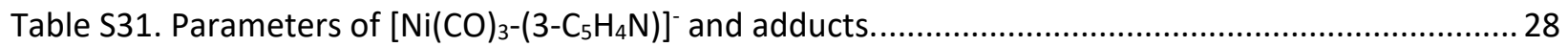

Table S32. Formation energies and enthalpies of Lewis acid adducts of $\left[\mathrm{Ni}(\mathrm{CO})_{3}-\left(3-\mathrm{C}_{5} \mathrm{H}_{4} \mathrm{~N}\right)\right]^{-} \ldots \ldots \ldots \ldots \ldots . . . . . .28$

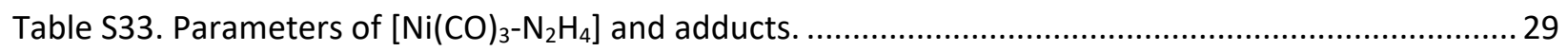

Table S34. Formation energies and enthalpies of Lewis acid adducts of $\left[\mathrm{Ni}(\mathrm{CO})_{3}-\mathrm{N}_{2} \mathrm{H}_{4}\right] \ldots \ldots \ldots \ldots \ldots \ldots \ldots . . . . . . . . . . .29$

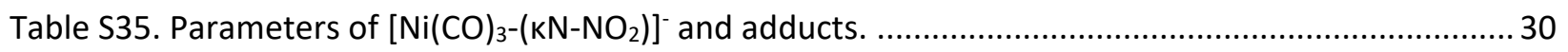

Table S36. Formation energies and enthalpies of Lewis acid adducts of $\left[\mathrm{Ni}(\mathrm{CO})_{3}-\left(\mathrm{KN}^{\left.-\mathrm{NO}_{2}\right)}\right]^{-} \ldots \ldots \ldots \ldots \ldots . . . . . . . .30\right.$

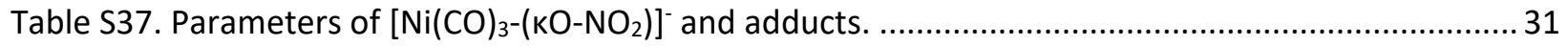

Table S38. Formation energies and enthalpies of Lewis acid adducts of $\left[\mathrm{Ni}(\mathrm{CO})_{3}-\left(\mathrm{KO}^{-} \mathrm{NO}_{2}\right)\right]^{-} \ldots \ldots \ldots \ldots \ldots . . . . . . . .31$

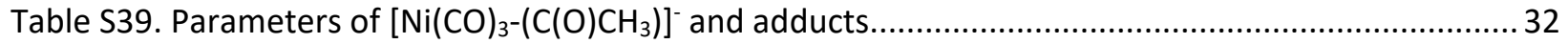

Table S40. Formation energies and enthalpies of Lewis acid adducts of $\left[\mathrm{Ni}(\mathrm{CO})_{3}-\left(\mathrm{C}(\mathrm{O}) \mathrm{CH}_{3}\right)\right]^{-} \ldots \ldots \ldots \ldots . \ldots . \ldots . \ldots$

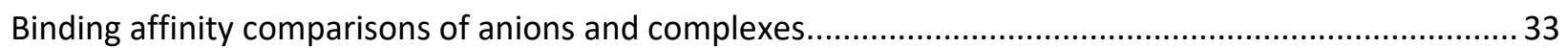

Figure S7. Lewis acid binding free energy to $\left[\mathrm{Ni}(\mathrm{CO})_{3-\mathrm{X}}\right]^{-}$as a function of $\mathrm{X}$ anion affinity......................33

Figure S8. Lewis acid binding enthalpies to $\left[\mathrm{Ni}(\mathrm{CO})_{3}-\mathrm{X}\right]^{-}$as a function of Lewis acidity. The gray shaded areas reflect where Lewis acid interactions were insufficiently strong to model for complexes and any

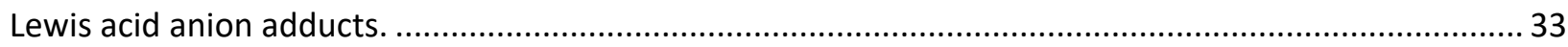

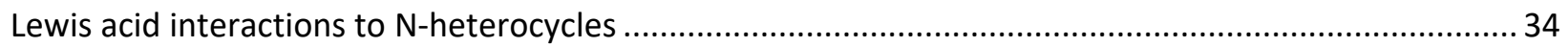

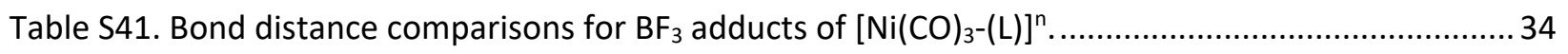

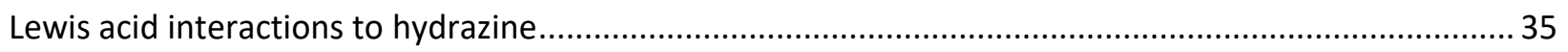

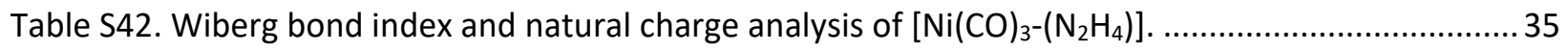

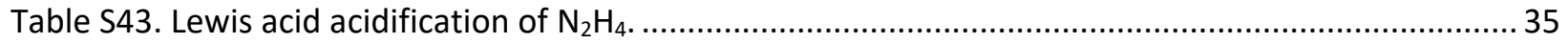

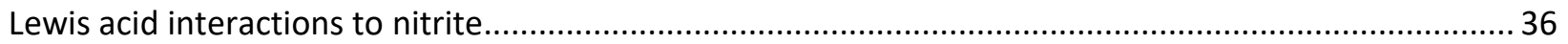

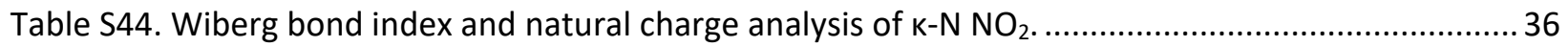

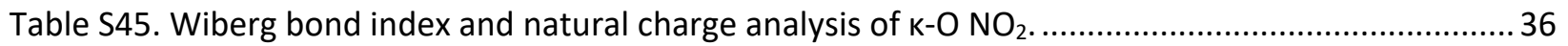


Figure S9. Wiberg bond indices of nitro (left) and nitrito (right) configuration of Lewis acid adducts of

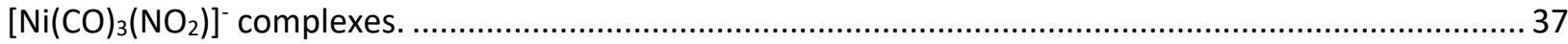

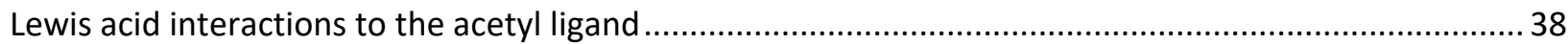

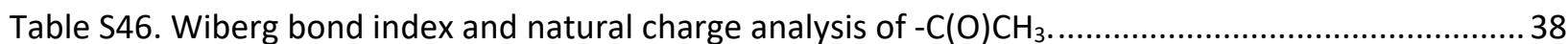

Figure S10. Wiberg bond indices of the acetyl ligand of $\left[\mathrm{Ni}(\mathrm{CO})_{3}\left(\mathrm{C}(\mathrm{O}) \mathrm{CH}_{3}\right)\right]^{-}$and corresponding Lewis acid adducts.

Figure S11. Molecular orbital renderings and energies $(\mathrm{eV})$ for $\left[\mathrm{Ni}(\mathrm{CO})_{3}\left(\mathrm{C}(\mathrm{O}) \mathrm{CH}_{3}\right)\right]^{-}\left(\right.$left) and $\mathrm{BF}_{3}$ adduct (right) with corresponding fragment orbitals in gray. 39

Figure S12. Molecular orbital renderings and energies (eV) for acetone (left) and $\mathrm{BF}_{3}$ adduct (right)......40

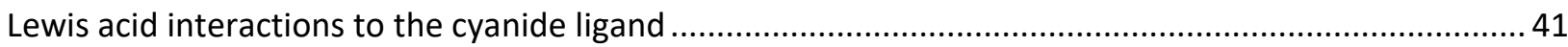

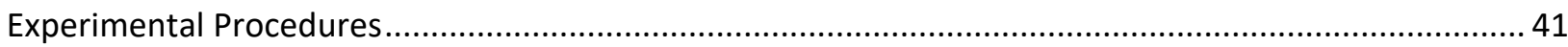

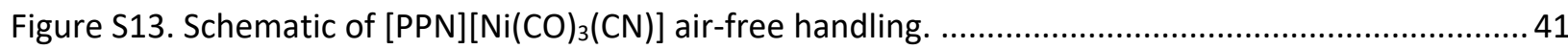

Lewis acid stock solutions ( $20 \mu \mathrm{L}, 0.26 \mathrm{M}, 0.05 \mathrm{mmol}$ ) in $\mathrm{CH}_{2} \mathrm{Cl}_{2}$ were combined with $0.3 \mathrm{~mL}$ aliquots of an $0.0175 \mathrm{M}$ 41

Figure S14. IR spectra $\left(\mathrm{CH}_{2} \mathrm{Cl}_{2}\right)$ of $\left[\mathrm{PPh}_{4}\right]\left[\mathrm{Ni}(\mathrm{CN})(\mathrm{CO})_{3}\right]$ with specified Lewis acid..................................42

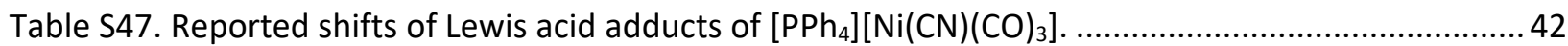

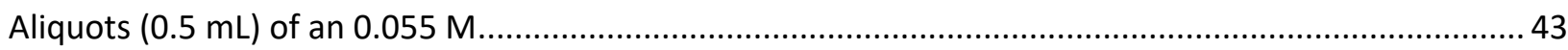

Table S48. Acceptor number data for measurement of Lewis acidity.................................................. 43

Computational analysis of Lewis acid effects to cyanide.........................................................................4 44

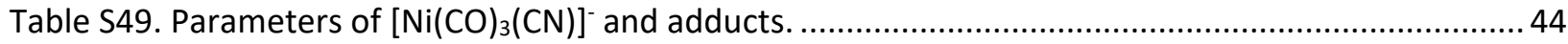

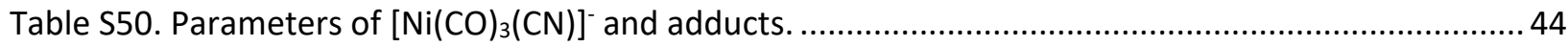

Table S51. Parameters of $\left[\mathrm{Ni}(\mathrm{CO})_{3}(\mathrm{NC})\right]^{-}$isomer and selected adducts...............................................45

Figure S15. Representative experimental solution spectra and calculated IR spectra...........................45

Table S52. Bond distances and natural charge analysis of $\mathrm{CN}^{-}$of $\left[\mathrm{Ni}(\mathrm{CO})_{3}(\mathrm{CN})\right]^{-}$and adducts.................46

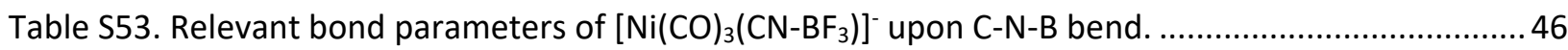

Figure S16. Ni-C (black) and C-N (blue) Wiberg bond indices of $\left[\mathrm{Ni}(\mathrm{CO})_{3}\left(\mathrm{CN}-\mathrm{BF}_{3}\right)\right]^{-}$as a function of C-N-B angle from a relaxed potential energy scan.

Figure S17. Relative energies $(\mathrm{kcal} / \mathrm{mol})$ of $\left[\mathrm{Ni}(\mathrm{CO})_{3}\left(\mathrm{CN}_{-}-\mathrm{BF}_{3}\right)\right]^{-}$as a function of $\mathrm{C}-\mathrm{N}-\mathrm{B}$ angle from a relaxed potential energy scan

Table S54. Relevant orbital energies $(\mathrm{eV})$ of $\left[\mathrm{Ni}(\mathrm{CO})_{3}(\mathrm{CN})\right]^{-},\left[\mathrm{Ni}(\mathrm{CO})_{3}\left(\mathrm{CN}-\mathrm{BF}_{3}\right)\right]^{-}$, $\left[\mathrm{Ni}(\mathrm{CO})_{3}\left(\mathrm{CN}-\mathrm{BF}_{3}\right)\right]^{-}$with a $120^{\circ} \mathrm{C}-\mathrm{N}-\mathrm{B}$ bend.

Figure S18. Orbital renderings of representative frontier orbitals of $\left[\mathrm{Ni}(\mathrm{CO})_{3}(\mathrm{CN})\right]^{-}$(left) and $\mathrm{BF}_{3}$ adduct (center) and $\mathrm{BF}_{3}$ adduct at $120^{\circ} \mathrm{CNB}$ angle (right). 


\section{Computational Analysis}

\section{General Considerations.}

DFT calculations were performed using the Gaussian 16 revA03-avx2 software package. ${ }^{1}$ Charges and Wiberg bond indices were determined using NBO version 3.0. ${ }^{2}$ To calculate electronic parameters we applied a previously reported method $^{3-4}$ using the mPW1PW91 functional ${ }^{5}$ and the $6-311+G(d, p)$ basis set for all atoms except $\mathrm{Ni}$, which was treated with $6-311+\mathrm{G}(2 \mathrm{~d}) \cdot{ }^{6-7}$ In accordance with the adopted method, ${ }^{3-}$ ${ }^{4}$ the evaluated the symmetric $v_{\text {co }}$ vibration was analyzed and a correction factor (multiply value by 0.954 ) was applied to the stretch. Vibrations for hydride are reported for the ${ }^{3} \mathrm{H}$ isotope to mitigate coupling of the $\mathrm{Ni}-\mathrm{H}$ and $\mathrm{CO}$ vibrational modes. Structures were confirmed minima by the absence of imaginary frequencies. Energies were determined by applying the free energy and enthalpy corrections from the frequency calculations to the scf energies from single point calculations of the optimized geometries with a polarizable continuum model of $\mathrm{CH}_{2} \mathrm{Cl}_{2}$ solvent correction were used for reported free energies. The single point calculations were used in electronic structure, charge, and Wiberg bond index analysis. For determination of Brønsted acidity (see below), an acetonitrile solvation model was used and the calculated $\mathrm{p} K_{\mathrm{a}}$ values calibrated to those of pyridine/pyridinium. ${ }^{8}$ Alternative input configurations of $[\mathrm{Ni}(\mathrm{CO})-\mathrm{L}-\mathrm{LA}]^{\mathrm{n}}$ of L-LA for symmetric $\mathrm{LA}\left(\mathrm{BF}_{3}, \mathrm{BMe}_{3}\right.$ etc.) would converge to identical structures, while lower symmetry Lewis acids, $\mathrm{B}(\mathrm{OPh}) \mathrm{Me}_{2}$ resulted in alternative local minima. However, the alternative configurations of L-LA (PHPh-B(OPh) $\mathrm{Me}_{2}{ }^{-}$, and $4-\mathrm{C}_{4} \mathrm{H}_{4} \mathrm{~N}_{2}-\mathrm{B}(\mathrm{OPh}) \mathrm{Me}_{2}$ ) were found to not provide significant changes to $v_{\mathrm{co}}\left(<0.5 \mathrm{~cm}^{-1}\right)$ or energies $(<0.1 \mathrm{kcal} / \mathrm{mol})$. In all cases, lower energy structures were obtained with the -OPh group oriented away from the B-L bond to minimize steric interactions. This input configuration was then adopted as an input for pertinent optimizations of $\mathrm{B}(\mathrm{OPh}) \mathrm{Me}_{2}$ and $\mathrm{BF}_{2} \mathrm{Ph}$. 


\section{Computational Summary}

In Figures S1-S4 parameterized values for electronic shifts and association energies are presented. In Figures S1-S3, gray filled boxes were not modeled to bind.

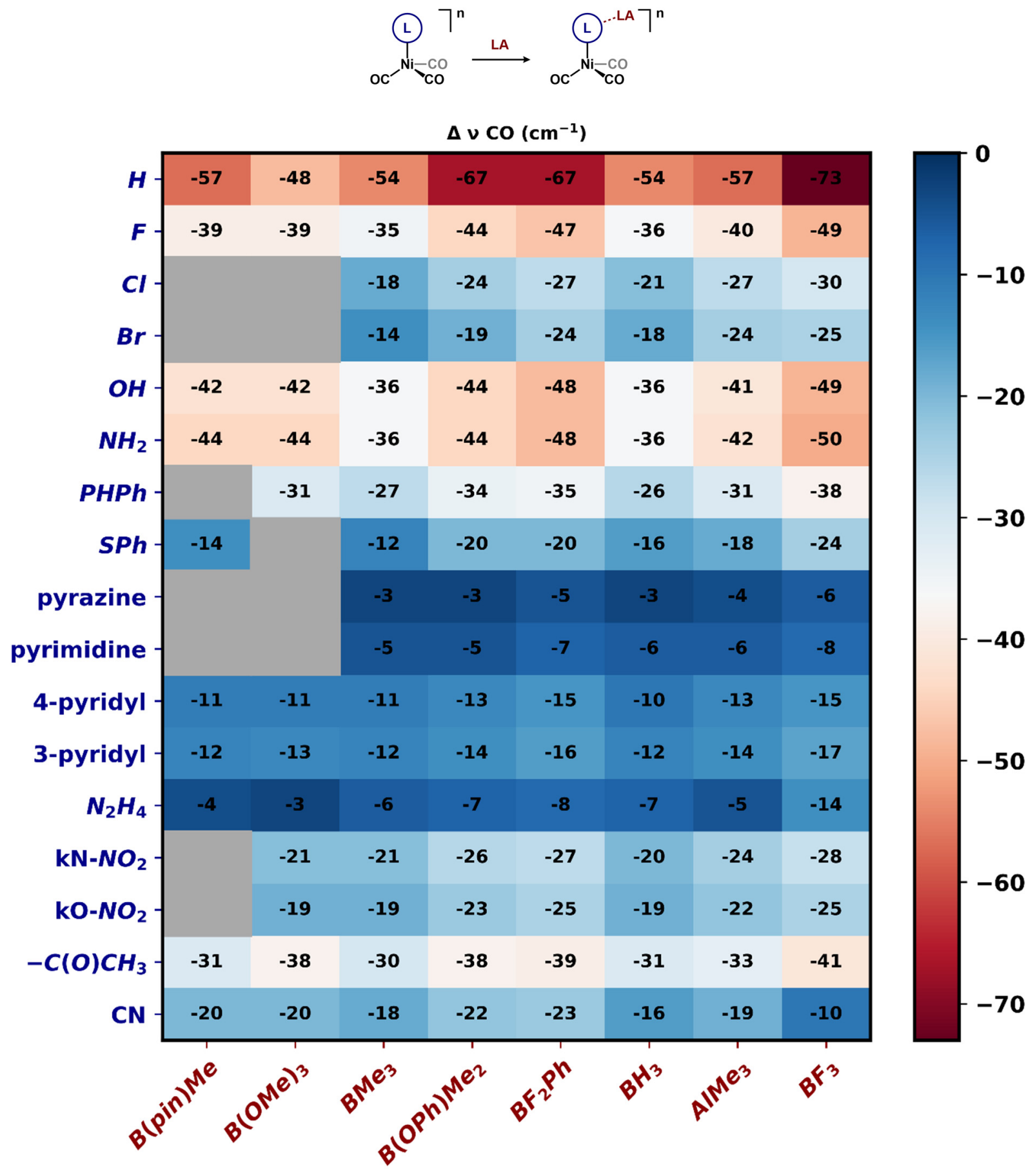

Figure S1. Table of calibrated $\Delta \mathrm{v}_{\mathrm{CO}(\mathrm{a})}\left(\mathrm{cm}^{-1}\right)$ for the series of ligands and Lewis acid adducts. 


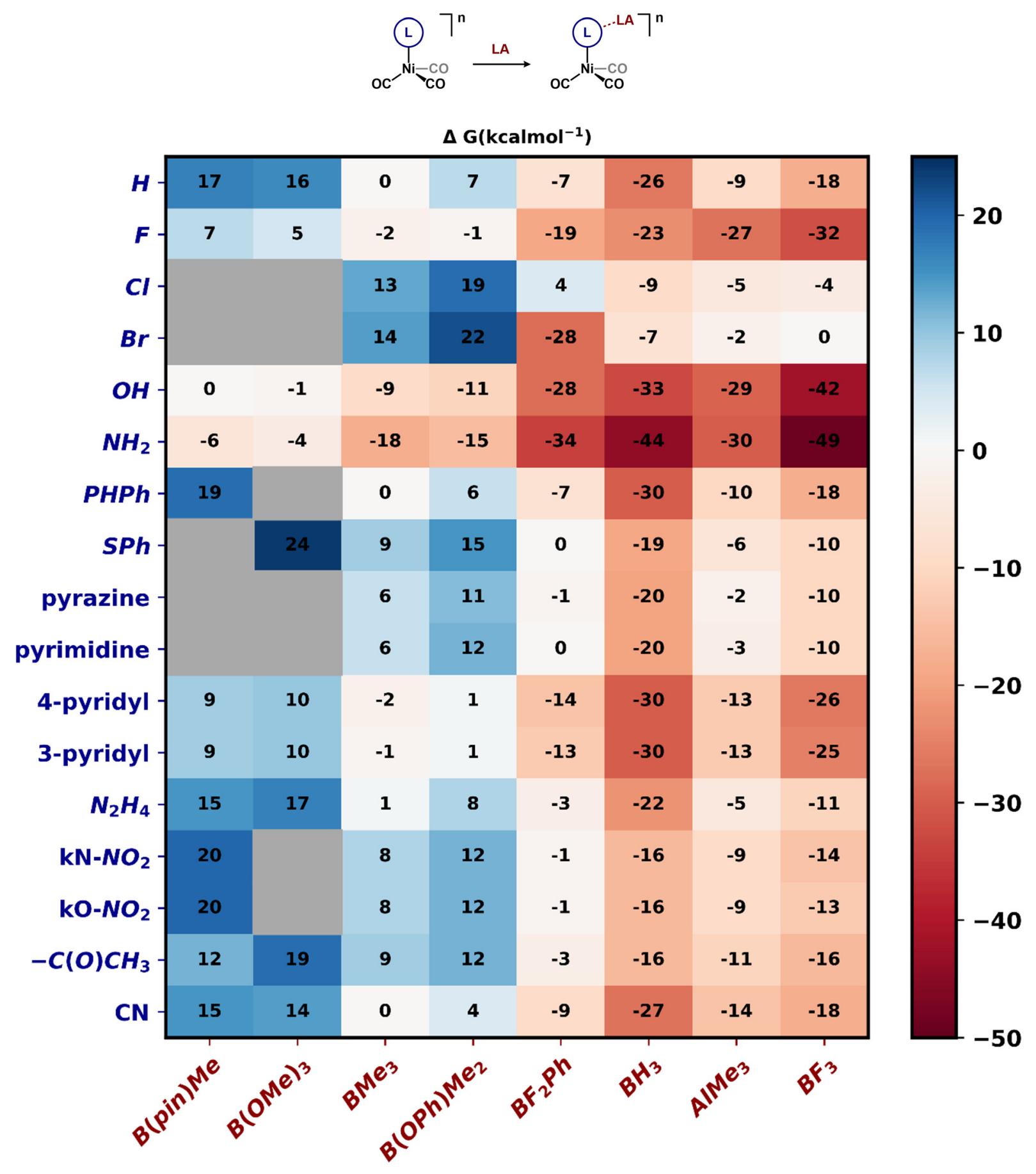

Figure S2. Table of $\Delta \mathrm{G}(\mathrm{kcal} / \mathrm{mol})$ for the series of ligands and Lewis acid adducts. 


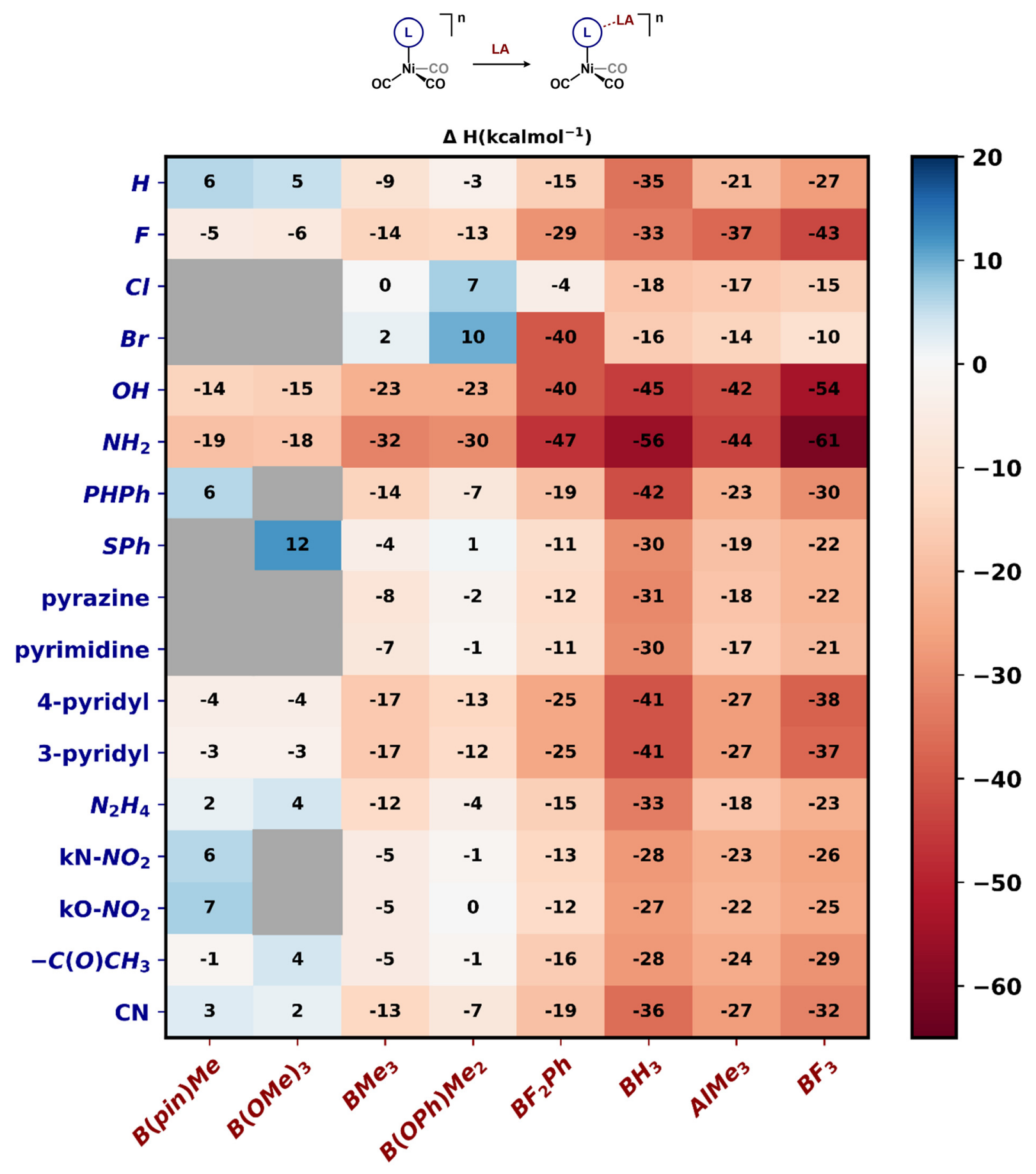

Figure S3. Table of $\Delta \mathrm{H}(\mathrm{kcal} / \mathrm{mol})$ for the series of ligands and Lewis acid adducts. 


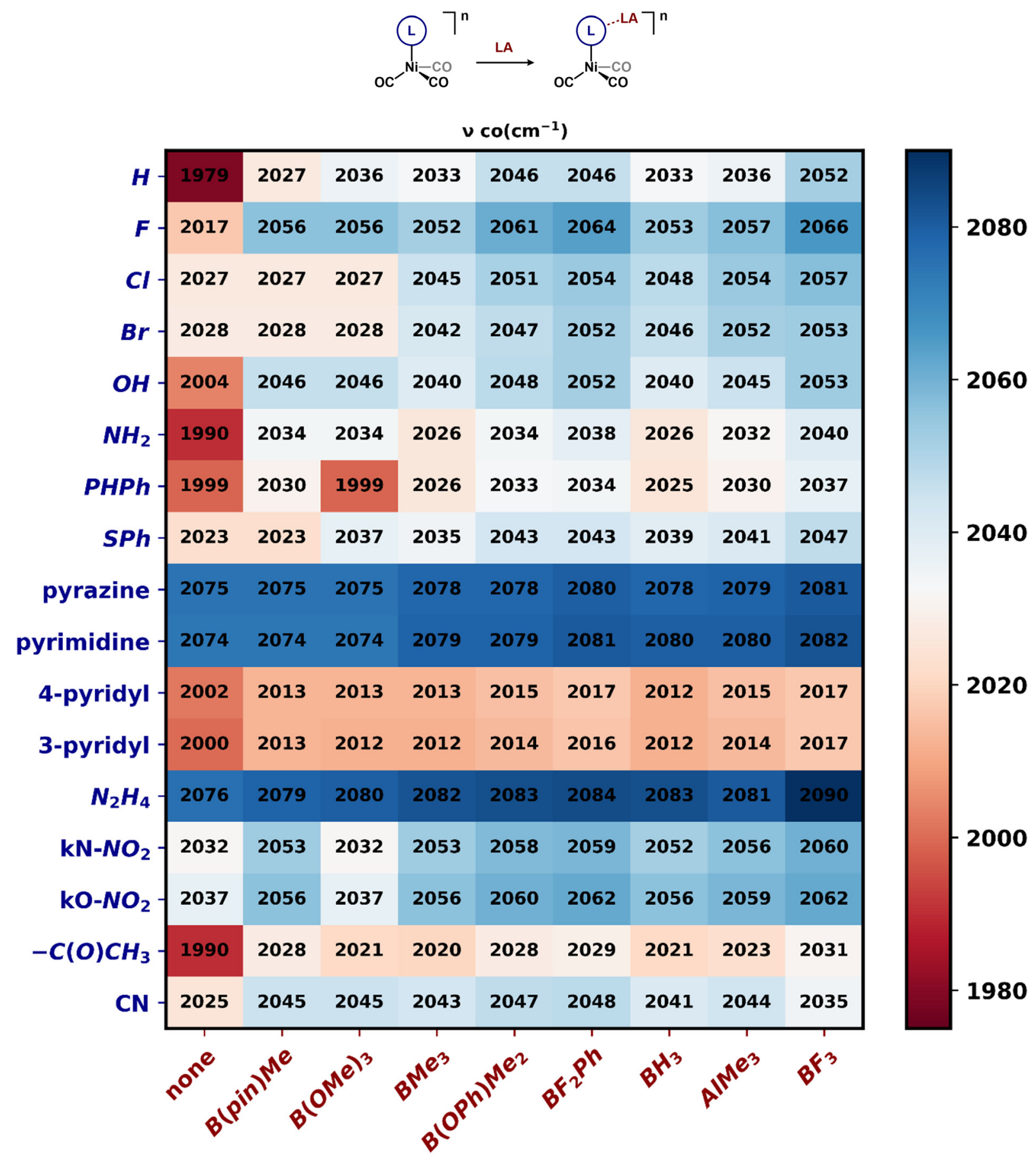

Figure S4. Table of calibrated $\mathrm{v}_{\mathrm{CO}(\mathrm{a} 1)}\left(\mathrm{cm}^{-1}\right)$ for the series of ligands and Lewis acid adducts. 


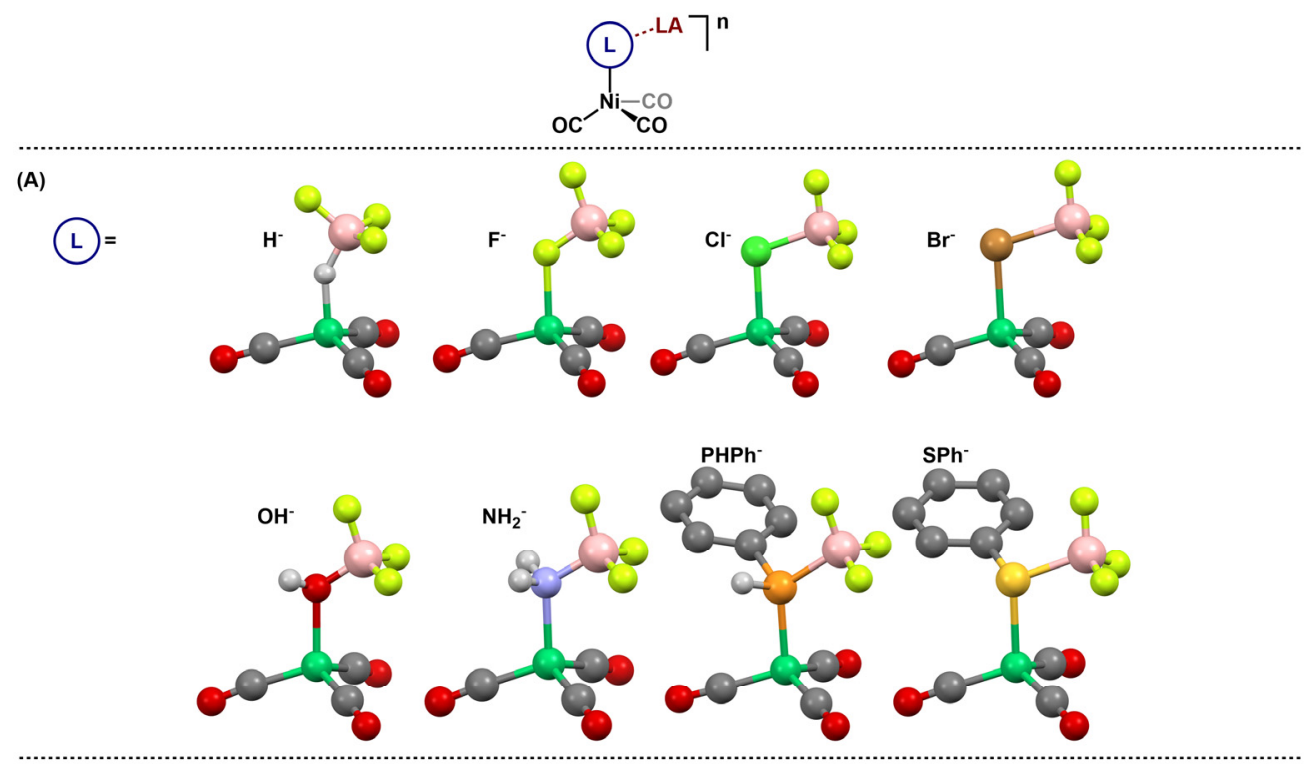

(B)

(L) $=$
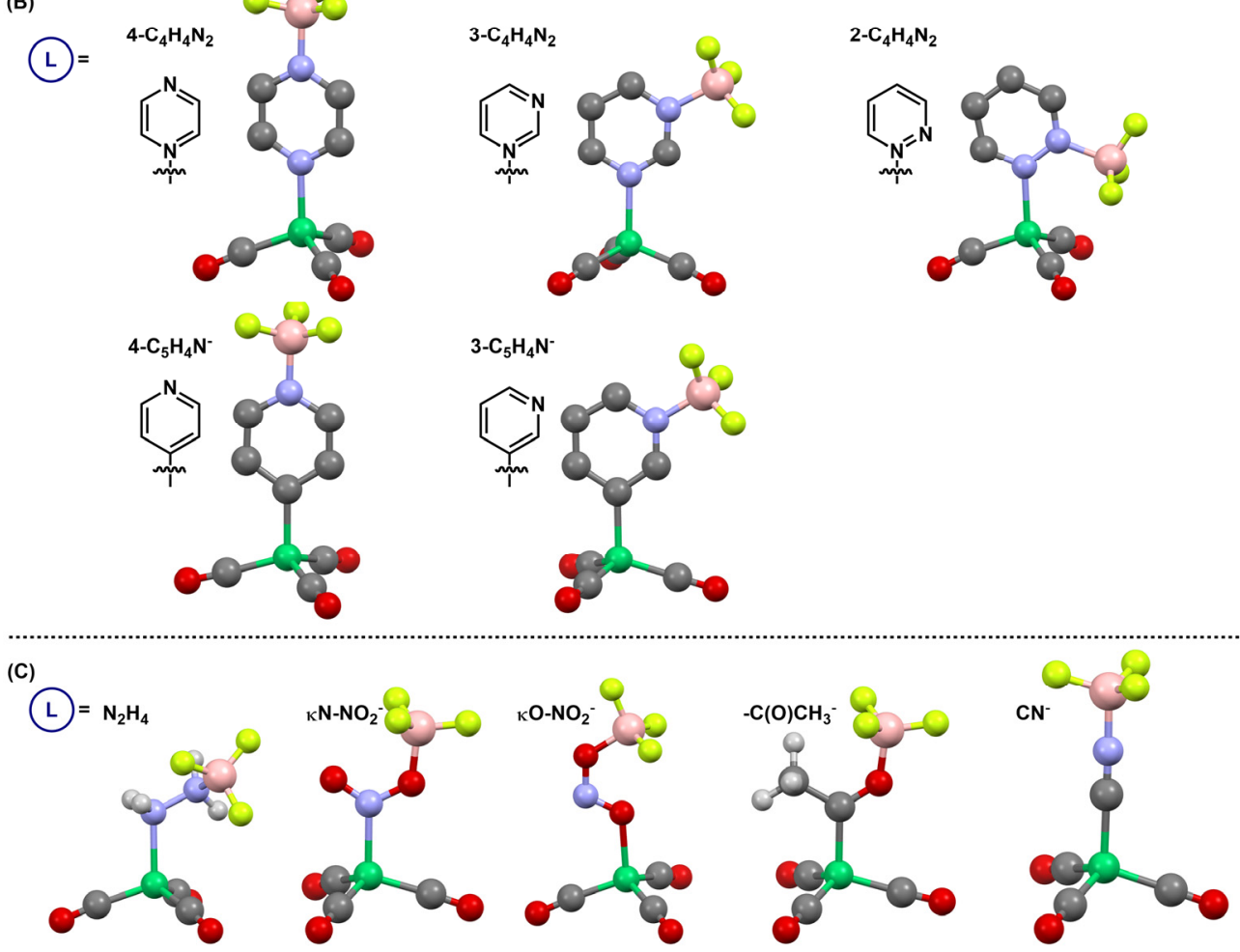

Figure S5. Representative rendered structures of $\mathrm{BF}_{3}$ adducts of $\left[\mathrm{Ni}(\mathrm{CO})_{3}-\mathrm{L}\right]^{\mathrm{n}}$ complexes of $(\mathrm{A}) \mathrm{X}$-type ligands, (B) N-heterocycle ligands, and (C) small molecules. 


\section{Application to other systems}

With respect to binding energies ( $\Delta \mathrm{G}$; Figure $\mathrm{S} 2$ ), values in blue are not anticipated to favorably bind and may require excess Lewis acid to bias association. While effective concentration behavior is expected to be dependent the intramolecular tether, ${ }^{9}$ For complexation of flexible linkers, such as the ones described, we consider a model where the dominant contribution for the effective concentration is entropy driven ${ }^{9}$ and thus better modeled as a $\Delta \mathrm{H}$ term. With respect to binding enthalpies ( $\Delta \mathrm{H}$; Figure S3), values in blue are not anticipated to favorably bind for an intramolecular system while those in red should be amenable. For the simple intermolecular binding model, the $\mathrm{T} \Delta \mathrm{S}$ contribution is relatively constant $(12.3 \pm 1.6$ $\mathrm{kcal} / \mathrm{mol})$.

Within some range of uncertainty ( $\sim \mathrm{kcal} / \mathrm{mol})$ for these binding energies these values approximate the solution behavior.
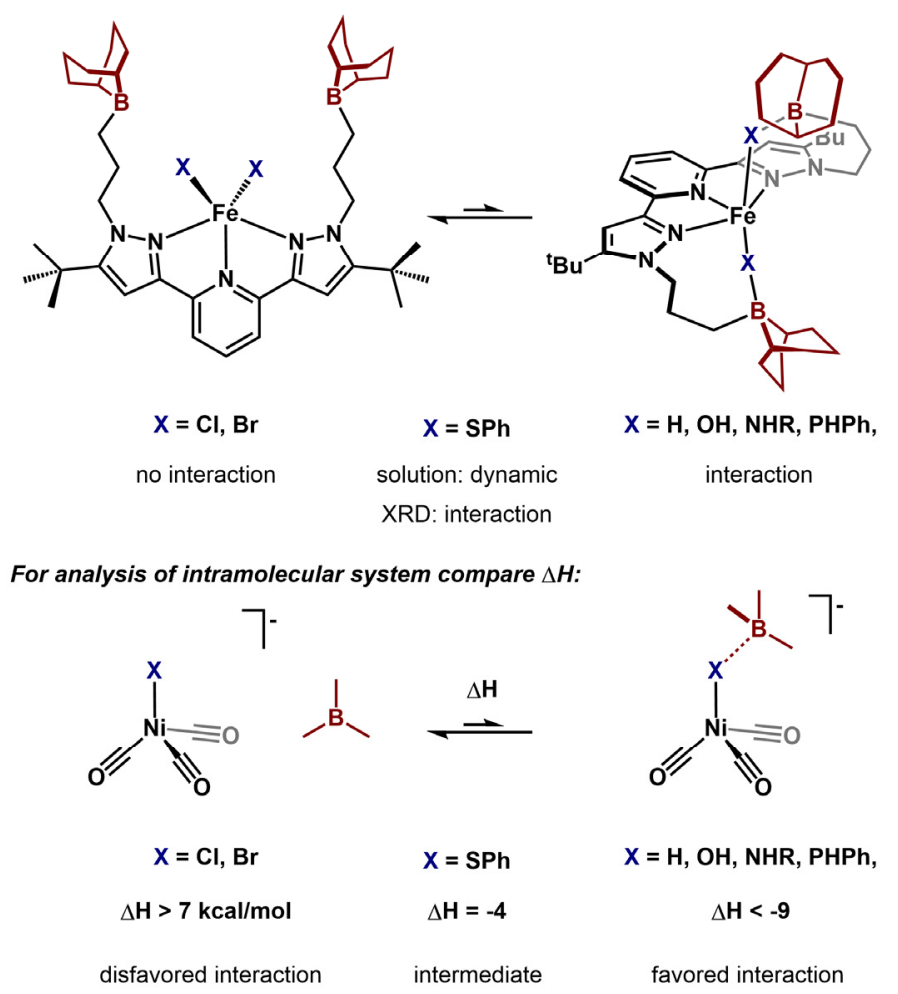

Figure S6. Lewis acid behavior of $\mathrm{Fe}(\mathrm{X})_{2}\left({ }^{\mathrm{BBN}} \mathrm{PDP}^{\mathrm{tBu}}\right)$ complexes $^{10}$ and parameters from $\left[\mathrm{Ni}(\mathrm{CO})_{3} \mathrm{X}\right]^{-}$analysis for $\mathrm{B}\left(\mathrm{CH}_{3}\right)_{3}$. 
Tabulated Parameters of Lewis acid interactions

Table S1. Calculated energies of optimized Lewis acids.

\begin{tabular}{llll}
\hline Lewis acid & $\begin{array}{l}\mathbf{G}_{\text {corr }} \\
\text { (Hartree) }\end{array}$ & $\begin{array}{l}\mathbf{H}_{\text {corr }} \\
\text { (Hartree) }\end{array}$ & $\begin{array}{l}\text { SCF }\left(\mathbf{C H}_{2} \mathbf{C l}_{2}\right) \\
\text { (Hartree) }\end{array}$ \\
\hline $\mathrm{B}\left(\mathrm{O}^{\prime} \mathrm{Pr}\right)_{3}$ & 0.25586 & 0.320753 & -606.31302789 \\
$\mathrm{BBu}$ & 0.319825 & 0.39148 & -498.43098343 \\
$\mathrm{BBN}(\mathrm{OPh})$ & 0.265476 & 0.321747 & -645.07957723 \\
$\mathrm{~B}(\mathrm{OPh})_{3}$ & 0.239999 & 0.310786 & -945.62131602 \\
$\mathrm{~B}(\mathrm{Ph})_{3}$ & 0.234356 & 0.294332 & -719.82265230 \\
$\mathrm{~B}\left(\mathrm{C}_{6} \mathrm{H}_{3} \mathrm{~F}_{2}\right)_{3}$ & 0.176786 & 0.249831 & -1315.29441493 \\
$\mathrm{~B}\left(\mathrm{C}_{6} \mathrm{~F}_{5}\right)_{3}$ & 0.094116 & 0.185609 & -2208.40159450 \\
$\mathrm{Al}\left(\mathrm{CH}_{3}\right)_{3}$ & 0.071343 & 0.115636 & -362.20134310 \\
\hline $\mathrm{B}\left(\mathrm{OCH}_{3}\right)_{3}$ & 0.097062 & 0.144053 & -370.39521468 \\
$\mathrm{Bpin}\left(\mathrm{CH}_{3}\right)$ & 0.182357 & 0.231158 & -451.21704579 \\
$\mathrm{~B}\left(\mathrm{CH}_{3}\right)_{3}$ & 0.082120 & 0.120964 & -144.61059548 \\
$\mathrm{~B}\left(\mathrm{CH}_{3}\right)_{2}(\mathrm{OPh})$ & 0.135763 & 0.184672 & -411.61967581 \\
$\mathrm{BF}{ }_{2}(\mathrm{Ph})$ & 0.068591 & 0.109377 & -456.33463471 \\
$\mathrm{BH}{ }_{3}$ & 0.006917 & 0.029991 & -26.60214108 \\
$\mathrm{Al}\left(\mathrm{CH}_{3}\right)_{3}$ & 0.071343 & 0.115636 & -362.20134310 \\
$\mathrm{BF}{ }_{3}$ & -0.013873 & 0.016744 & -324.57735985 \\
$\mathrm{~B}\left(\mathrm{OCH}_{3}\right)_{3}$ & 0.097062 & 0.144053 & -370.39521468 \\
$\mathrm{Bpin}_{3}\left(\mathrm{CH}_{3}\right)$ & 0.182357 & 0.231158 & -451.21704579 \\
\hline
\end{tabular}


Table S2. Calculated energies for Fluoride Ion Affinity Lewis acid scale.

\begin{tabular}{|c|c|c|c|c|c|}
\hline \multirow[b]{2}{*}{ Lewis acid } & \multirow[b]{2}{*}{$\begin{array}{l}\mathbf{G}_{\text {corr }} \\
\text { (Hartree) }\end{array}$} & \multirow[b]{2}{*}{$\begin{array}{l}\mathbf{H}_{\text {Corr }} \\
\text { (Hartree) }\end{array}$} & \multirow[b]{2}{*}{$\begin{array}{l}\text { SCF }\left(\mathrm{CH}_{2} \mathrm{Cl}_{2}\right) \\
\text { (Hartree) }\end{array}$} & \multicolumn{2}{|c|}{ Fluoride ion Affinity } \\
\hline & & & & $\Delta \mathrm{G}(\mathrm{kcal} / \mathrm{mol})$ & $\Delta \mathrm{H}(\mathrm{kcal} / \mathrm{mol})$ \\
\hline Fluoride & -0.01416 & 0.00236 & -99.97847665 & & \\
\hline $\mathrm{B}\left(\mathrm{O}^{i} \mathrm{Pr}\right)_{3}$ & 0.254624 & 0.320811 & -706.31010847 & -3.6 & -13.1 \\
\hline $\mathrm{BBu}_{3}$ & 0.323346 & 0.392386 & -598.44399467 & -10.6 & -22.6 \\
\hline $\mathrm{BBN}(\mathrm{OPh})$ & 0.266194 & 0.322961 & -745.09885784 & -26.3 & -26.3 \\
\hline $\mathrm{B}(\mathrm{OPh})_{3}$ & 0.241706 & 0.312392 & -1045.65704769 & -26.0 & -36.4 \\
\hline $\mathrm{B}(\mathrm{Ph})_{3}$ & 0.23179 & 0.295727 & -819.85023672 & -31.4 & -31.4 \\
\hline $\mathrm{B}\left(\mathrm{C}_{6} \mathrm{H}_{3} \mathrm{~F}_{2}\right)_{3}$ & 0.176591 & 0.251866 & -1415.33454054 & -29.9 & -38.9 \\
\hline $\mathrm{B}\left(\mathrm{C}_{6} \mathrm{~F}_{5}\right)_{3}$ & 0.093979 & 0.188135 & -2308.46435852 & -44.1 & -52.7 \\
\hline $\mathrm{Al}\left(\mathrm{CH}_{3}\right)_{3}$ & 0.073549 & 0.117651 & -462.25563874 & -37.3 & -47.8 \\
\hline $\mathrm{B}\left(\mathrm{OCH}_{3}\right)_{3}$ & 0.096692 & 0.144232 & -470.39989214 & -7.8 & -17.8 \\
\hline $\operatorname{Bpin}\left(\mathrm{CH}_{3}\right)$ & 0.181841 & 0.231895 & -551.21930289 & -6.4 & -15.9 \\
\hline $\mathrm{B}\left(\mathrm{CH}_{3}\right)_{3}$ & 0.084694 & 0.123130 & -244.62879829 & -14.4 & -25.0 \\
\hline $\mathrm{B}\left(\mathrm{CH}_{3}\right)_{2}(\mathrm{OPh})$ & 0.136810 & 0.186417 & -511.63755418 & -15.2 & -25.1 \\
\hline $\mathrm{BF}_{2}(\mathrm{Ph})$ & 0.066616 & 0.111287 & -556.38033632 & -34.5 & -42.4 \\
\hline $\mathrm{BH}_{3}$ & 0.006777 & 0.033455 & -126.65377235 & -37.1 & -45.2 \\
\hline $\mathrm{Al}\left(\mathrm{CH}_{3}\right)_{3}$ & 0.073549 & 0.117651 & -462.25563874 & -37.3 & -47.8 \\
\hline $\mathrm{BF}_{3}$ & -0.013726 & 0.019291 & -424.64719079 & -48.3 & -57.2 \\
\hline
\end{tabular}

Table S3. Calculated energies for hydride ion affinity.

\begin{tabular}{|c|c|c|c|c|c|}
\hline \multirow[b]{2}{*}{ Lewis acid } & \multirow[b]{2}{*}{$\begin{array}{l}G_{\text {corr }} \\
\text { (Hartree) }\end{array}$} & \multirow[b]{2}{*}{$\begin{array}{l}\mathrm{H}_{\text {corr }} \\
\text { (Hartree) }\end{array}$} & \multicolumn{3}{|c|}{ hydride ion Affinity } \\
\hline & & & $\begin{array}{l}\mathrm{SCF}\left(\mathrm{CH}_{2} \mathrm{Cl}_{2}\right) \\
\text { (Hartree) }\end{array}$ & $\Delta \mathrm{G}$ (kcal/mol) & $\Delta \mathrm{H}(\mathrm{kcal} / \mathrm{mol})$ \\
\hline hydride & -0.010000 & 0.002360 & -0.63356281 & & \\
\hline $\mathrm{B}\left(\mathrm{OCH}_{3}\right)_{3}$ & 0.103640 & 0.149048 & -371.06630849 & -13.1 & -21.9 \\
\hline $\mathrm{Bpin}\left(\mathrm{CH}_{3}\right)$ & 0.188518 & 0.236684 & -451.88297635 & -10.2 & -18.3 \\
\hline $\mathrm{B}\left(\mathrm{CH}_{3}\right)_{3}$ & 0.091960 & 0.127901 & -145.30388201 & -25.0 & -34.6 \\
\hline $\mathrm{B}\left(\mathrm{CH}_{3}\right)_{2}(\mathrm{OPh})$ & 0.144269 & 0.191615 & -412.30878826 & -23.2 & -32.0 \\
\hline $\mathrm{BF}_{2}(\mathrm{Ph})$ & 0.074078 & 0.116375 & -457.04700405 & -39.7 & -46.5 \\
\hline $\mathrm{BH}_{3}$ & 0.013371 & 0.037221 & -27.34562144 & -58.6 & -65.9 \\
\hline $\mathrm{Al}\left(\mathrm{CH}_{3}\right)_{3}$ & 0.079190 & 0.120606 & -362.90512348 & -32.8 & -42.4 \\
\hline $\mathrm{BF}_{3}$ & -0.006602 & 0.024619 & -325.31437400 & -54.0 & -61.4 \\
\hline
\end{tabular}


Table S4. Calculated energies for chloride ion affinity.

\begin{tabular}{|c|c|c|c|c|c|}
\hline \multirow[b]{2}{*}{ Lewis acid } & \multirow[b]{2}{*}{$\begin{array}{l}\mathbf{G}_{\text {corr }} \\
\text { (Hartree) }\end{array}$} & \multirow[b]{2}{*}{$\begin{array}{l}\mathbf{H}_{\text {Corr }} \\
\text { (Hartree) }\end{array}$} & \multirow[b]{2}{*}{$\begin{array}{l}\mathrm{SCF}\left(\mathrm{CH}_{2} \mathrm{Cl}_{2}\right) \\
\text { (Hartree) }\end{array}$} & \multicolumn{2}{|c|}{ chloride ion Affinity } \\
\hline & & & & $\Delta \mathrm{G}(\mathrm{kcal} / \mathrm{mol})$ & $\Delta \mathrm{H}(\mathrm{kcal} / \mathrm{mol})$ \\
\hline chloride & -0.015023 & 0.002360 & -460.40316512 & & \\
\hline $\mathrm{B}\left(\mathrm{OCH}_{3}\right)_{3}$ & - & & & & \\
\hline $\operatorname{Bpin}\left(\mathrm{CH}_{3}\right)$ & 0.180017 & 0.231779 & -911.60044187 & 20.3 & 11.3 \\
\hline $\mathrm{B}\left(\mathrm{CH}_{3}\right)_{3}$ & 0.083769 & 0.123281 & -605.01782321 & 7.9 & -2.6 \\
\hline $\mathrm{B}\left(\mathrm{CH}_{3}\right)_{2}(\mathrm{OPh})$ & 0.135445 & 0.186114 & -872.01660383 & 13.1 & 3.3 \\
\hline $\mathrm{BF}_{2}(\mathrm{Ph})$ & 0.064853 & 0.110567 & -916.75259225 & -2.2 & -10.0 \\
\hline $\mathrm{BH}_{3}$ & 0.005211 & 0.033403 & -487.04340188 & -15.5 & -23.2 \\
\hline $\mathrm{Al}\left(\mathrm{CH}_{3}\right)_{3}$ & 0.072173 & 0.117562 & -822.64107787 & -13.0 & -23.2 \\
\hline $\mathrm{BF}_{3}$ & -0.016160 & 0.018390 & -785.01457937 & -13.4 & -21.8 \\
\hline
\end{tabular}

Table S5. Calculated energies for bromide ion affinity.

\begin{tabular}{|c|c|c|c|c|c|}
\hline \multirow[b]{2}{*}{ Lewis acid } & \multirow[b]{2}{*}{$\begin{array}{l}\mathbf{G}_{\text {corr }} \\
\text { (Hartree) }\end{array}$} & \multirow[b]{2}{*}{$\begin{array}{l}\mathbf{H}_{\text {Corr }} \\
\text { (Hartree) }\end{array}$} & \multirow[b]{2}{*}{$\begin{array}{l}\mathrm{SCF}\left(\mathrm{CH}_{2} \mathrm{Cl}_{2}\right) \\
\text { (Hartree) }\end{array}$} & \multicolumn{2}{|c|}{ bromide ion Affinity } \\
\hline & & & & $\Delta \mathrm{G}(\mathrm{kcal} / \mathrm{mol})$ & $\Delta \mathrm{H}(\mathrm{kcal} / \mathrm{mol})$ \\
\hline bromide & -0.016176 & 0.002360 & -2574.48627674 & & \\
\hline $\mathrm{B}\left(\mathrm{OCH}_{3}\right)_{3}$ & - & & & & \\
\hline $\operatorname{Bpin}\left(\mathrm{CH}_{3}\right)$ & - & & & & \\
\hline $\mathrm{B}\left(\mathrm{CH}_{3}\right)_{3}$ & 0.082287 & 0.123324 & -2719.09550043 & 11.1 & 0.9 \\
\hline $\mathrm{B}\left(\mathrm{CH}_{3}\right)_{2}(\mathrm{OPh})$ & 0.133727 & 0.186031 & -2986.09185599 & 17.7 & 8.2 \\
\hline $\mathrm{BF}_{2}(\mathrm{Ph})$ & 0.063419 & 0.110440 & -3030.82670012 & 3.3 & -4.4 \\
\hline $\mathrm{BH}_{3}$ & 0.003668 & 0.033264 & -2601.12085182 & -12.2 & -19.8 \\
\hline $\mathrm{Al}\left(\mathrm{CH}_{3}\right)_{3}$ & 0.070848 & 0.117577 & -2936.71750248 & -8.9 & -19.0 \\
\hline $\mathrm{BF}_{3}$ & -0.017844 & 0.018219 & -2899.08684993 & -6.9 & -15.1 \\
\hline
\end{tabular}


Table S6. Parameters of $\left[\mathrm{Ni}(\mathrm{CO})_{3}-\mathrm{L}\right]^{\mathrm{n}}$ complexes

\begin{tabular}{|c|c|c|c|c|c|c|}
\hline $\mathbf{L}$ & $v \mathrm{CO}_{\mathrm{a} 1}\left(\mathrm{~cm}^{-1}\right)$ & $\begin{array}{l}\mathrm{vCO}_{\mathrm{a} 1}\left(\mathrm{~cm}^{-1}\right) \\
\text { calibrated }\end{array}$ & Ave C-O (Å) & G (Hartree) & H (Hartree) & $\begin{array}{l}\mathrm{SCF}\left(\mathrm{CH}_{2} \mathrm{Cl}_{2}\right) \\
\text { (Hartree) }\end{array}$ \\
\hline $\mathrm{H}^{-}$ & 2077 & 1981 & 1.156 & -0.004993 & 0.038749 & -1849.12373402 \\
\hline $\mathrm{H}^{-}\left({ }^{3} \mathrm{H}\right)$ & 2074 & 1979 & & & & \\
\hline $\mathrm{F}^{-}$ & 2114 & 2017 & 1.150 & -0.013335 & 0.035154 & -1948.41979264 \\
\hline $\mathrm{Cl}^{-}$ & 2125 & 2027 & 1.148 & -0.014070 & 0.035194 & -2308.83940920 \\
\hline $\mathrm{Br}^{-}$ & 2126 & 2028 & 1.147 & -0.015198 & 0.035184 & -4422.92265208 \\
\hline $\mathrm{OH}^{-}$ & 2101 & 2004 & 1.151 & -0.003139 & 0.046845 & -1924.36991939 \\
\hline $\mathrm{NH}_{2}^{-}$ & 2086 & 1990 & 1.153 & 0.009525 & 0.059164 & -1904.48182378 \\
\hline $\mathrm{PHPh}^{-}$ & 2096 & 1999 & 1.150 & 0.076934 & 0.140248 & -2422.18566015 \\
\hline $\mathrm{SPh}^{-}$ & 2120 & 2023 & 1.148 & 0.068998 & 0.131318 & -2478.45106069 \\
\hline pyrazine & 2175 & 2075 & 1.140 & 0.057633 & 0.117221 & -2112.75755485 \\
\hline pyrimidine & 2174 & 2074 & 1.140 & 0.057439 & 0.117424 & -2112.76545822 \\
\hline pyridazine & 2173 & 2073 & 1.140 & 0.057537 & 0.116682 & -2112.73344913 \\
\hline 4-pyridine & 2098 & 2002 & 1.151 & 0.056697 & 0.115749 & -2096.21623653 \\
\hline 3-pyridine & 2096 & 2000 & 1.151 & 0.056822 & 0.115689 & -2096.21433176 \\
\hline $\mathrm{N}_{2} \mathrm{H}_{4}$ & 2176 & 2076 & 1.140 & 0.040757 & 0.093606 & -1960.32574066 \\
\hline$-\mathrm{NO}_{2}^{-}$ & 2130 & 2032 & 1.147 & -0.007841 & 0.047095 & -2053.70055266 \\
\hline$-\mathrm{O}_{2} \mathrm{~N}^{-}$ & 2135 & 2037 & 1.147 & -0.008450 & 0.046449 & -2053.69126967 \\
\hline$-\mathrm{CO}\left(\mathrm{CH}_{3}\right)^{-}$ & 2086 & 1990 & 1.153 & 0.026644 & 0.082325 & -2001.77614518 \\
\hline $\mathrm{CN}^{-}$ & 2122 & 2025 & 1.148 & -0.006772 & 0.042821 & -1941.41385483 \\
\hline \multicolumn{7}{|l|}{ 4R-pyridine } \\
\hline $\mathrm{R}=-\mathrm{NO}_{2}$ & 2175 & 2075 & 1.140 & & & \\
\hline $\mathrm{R}=-\mathrm{CN}$ & 2174 & 2074 & 1.140 & & & \\
\hline $\mathrm{R}=-\mathrm{CF}_{3}$ & 2174 & 2074 & 1.140 & & & \\
\hline $\mathrm{R}=\mathrm{Br}$ & 2172 & 2072 & 1.141 & & & \\
\hline $\mathrm{R}=\mathrm{H}$ & 2170 & 2071 & 1.141 & & & \\
\hline $\mathrm{R}=\mathrm{CH}_{3}$ & 2169 & 2069 & 1.141 & & & \\
\hline $\mathrm{R}=\mathrm{OCH}_{3}$ & 2168 & 2069 & 1.141 & & & \\
\hline $\mathrm{R}=\mathrm{N}\left(\mathrm{CH}_{3}\right)_{2}$ & 2165 & 2065 & 1.142 & & & \\
\hline \multicolumn{7}{|l|}{ 4R-phenyl- } \\
\hline $\mathrm{R}=-\mathrm{NO}_{2}$ & 2104 & 2007 & 1.150 & & & \\
\hline $\mathrm{R}=-\mathrm{CN}$ & 2102 & 2005 & 1.150 & & & \\
\hline $\mathrm{R}=-\mathrm{CF}_{3}$ & 2100 & 2003 & 1.151 & & & \\
\hline $\mathrm{R}=\mathrm{Br}$ & 2097 & 2000 & 1.151 & & & \\
\hline $\mathrm{R}=\mathrm{H}$ & 2091 & 1995 & 1.152 & & & \\
\hline $\mathrm{R}=\mathrm{CH}_{3}$ & 2091 & 1994 & 1.152 & & & \\
\hline $\mathrm{R}=\mathrm{OCH}_{3}$ & 2091 & 1994 & 1.152 & & & \\
\hline $\mathrm{R}=\mathrm{N}\left(\mathrm{CH}_{3}\right)_{2}$ & 2089 & 1993 & 1.152 & & & \\
\hline
\end{tabular}


Table S7. Parameters of $\left[\mathrm{Ni}(\mathrm{CO})_{3}-\mathrm{H}\right]^{-}$and adducts with vibrational analysis for ${ }^{3} \mathrm{H}$.<smiles>O=[N+]([O-])[O-]</smiles>

\begin{tabular}{|c|c|c|c|c|c|c|}
\hline Lewis acid & $\begin{array}{l}\mathrm{vCO}_{\mathrm{a} 1} \\
\left(\mathrm{~cm}^{-1}\right)\end{array}$ & $\begin{array}{l}\mathrm{vCO}_{\mathrm{a} 1} \\
\left(\mathrm{~cm}^{-1}\right) \\
\text { calibrated }\end{array}$ & $\begin{array}{l}\text { Ave C-O } \\
\text { (Å) }\end{array}$ & $\mathrm{G}_{\text {corr }}$ (Hartree) & $\mathrm{H}_{\text {corr }}$ (Hartree) & $\begin{array}{l}\mathrm{SCF}\left(\mathrm{CH}_{2} \mathrm{Cl}_{2}\right) \\
\text { (Hartree) }\end{array}$ \\
\hline- & 2074 & 1979 & 1.156 & -0.004993 & 0.038749 & -1849.12373402 \\
\hline $\mathrm{B}\left(\mathrm{OCH}_{3}\right)_{3}$ & 2134 & 2036 & 1.146 & 0.110067 & 0.183520 & -2219.51061002 \\
\hline $\operatorname{Bpin}\left(\mathrm{CH}_{3}\right)$ & 2125 & 2027 & 1.147 & 0.195641 & 0.270922 & -2300.33188948 \\
\hline $\mathrm{B}\left(\mathrm{CH}_{3}\right)_{3}$ & 2131 & 2033 & 1.147 & 0.094372 & 0.162425 & -1993.75140311 \\
\hline $\mathrm{B}\left(\mathrm{CH}_{3}\right)_{2}(\mathrm{OPh})$ & 2145 & 2046 & 1.145 & 0.149630 & 0.225833 & -2260.75060824 \\
\hline $\mathrm{BF}_{2}(\mathrm{Ph})$ & 2145 & 2046 & 1.145 & 0.079167 & 0.150491 & -2305.48554355 \\
\hline $\mathrm{BH}_{3}$ & 2131 & 2033 & 1.147 & 0.020372 & 0.072702 & -1875.78611357 \\
\hline $\mathrm{Al}\left(\mathrm{CH}_{3}\right)_{3}$ & 2134 & 2036 & 1.146 & 0.087989 & 0.156698 & -2211.36106000 \\
\hline $\mathrm{BF}_{3}$ & 2150 & 2052 & 1.144 & -0.002152 & 0.058566 & -2173.74786825 \\
\hline
\end{tabular}

Table S8. Formation energies and enthalpies of Lewis acid adducts of $\left[\mathrm{Ni}(\mathrm{CO})_{3}-\mathrm{H}\right]^{-}$.

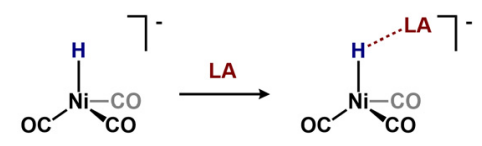

\begin{tabular}{|l|l|l|}
\hline Lewis acid & $\begin{array}{l}\Delta \mathbf{G} \\
\text { (kcal/mol) }\end{array}$ & $\begin{array}{l}\Delta \mathbf{H} \\
\text { (kcal/mol) }\end{array}$ \\
\hline $\mathrm{B}\left(\mathrm{OCH}_{3}\right)_{3}$ & 16.5 & 5.7 \\
\hline $\mathrm{Bpin}\left(\mathrm{CH}_{3}\right)$ & 17.0 & 6.2 \\
\hline $\mathrm{B}\left(\mathrm{CH}_{3}\right)_{3}$ & 0.1 & -9.0 \\
\hline $\mathrm{B}\left(\mathrm{CH}_{3}\right)_{2}(\mathrm{OPh})$ & 7.3 & -3.0 \\
\hline $\mathrm{BF}_{2}(\mathrm{Ph})$ & -7.3 & -15.6 \\
\hline $\mathrm{BH}_{3}$ & -26.2 & -35.3 \\
\hline $\mathrm{Al}_{3}\left(\mathrm{CH}_{3}\right)_{3}$ & -9.0 & -21.1 \\
\hline $\mathrm{BF}_{3}$ & -18.8 & -27.4 \\
\hline
\end{tabular}


Table S9. Parameters of $\left[\mathrm{Ni}(\mathrm{CO})_{3}-\mathrm{F}\right]^{-}$and adducts.

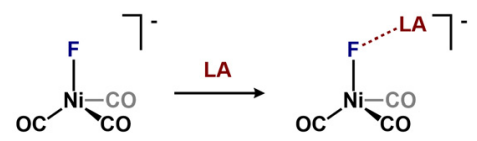

\begin{tabular}{|c|c|c|c|c|c|c|}
\hline Lewis acid & $\begin{array}{l}\mathrm{v} \mathrm{CO}_{\mathrm{a} 1} \\
\left(\mathrm{~cm}^{-1}\right)\end{array}$ & $\begin{array}{l}\mathrm{vCO}_{\mathrm{a} 1} \\
\left(\mathrm{~cm}^{-1}\right) \\
\text { calibrated }\end{array}$ & $\begin{array}{l}\text { Ave C-O } \\
\text { (Å) }\end{array}$ & $\mathrm{G}_{\text {corr }}($ Hartree) & $\mathbf{H}_{\text {corr }}($ Hartree $)$ & $\begin{array}{l}\mathrm{SCF}\left(\mathrm{CH}_{2} \mathrm{Cl}_{2}\right) \\
\text { (Hartree) }\end{array}$ \\
\hline- & 2114 & 2017 & 1.150 & -0.013335 & 0.035154 & -1948.41979264 \\
\hline $\mathrm{B}\left(\mathrm{OCH}_{3}\right)_{3}$ & 2155 & 2056 & 1.144 & 0.101973 & 0.179025 & -2318.82443463 \\
\hline $\mathrm{Bpin}\left(\mathrm{CH}_{3}\right)$ & 2155 & 2056 & 1.144 & 0.188614 & 0.266659 & -2399.64529755 \\
\hline $\mathrm{B}\left(\mathrm{CH}_{3}\right)_{3}$ & 2151 & 2052 & 1.145 & 0.089920 & 0.157903 & -2093.05549562 \\
\hline $\mathrm{B}\left(\mathrm{CH}_{3}\right)_{2}(\mathrm{OPh})$ & 2160 & 2061 & 1.144 & 0.142070 & 0.221014 & -2360.06173166 \\
\hline $\mathrm{BF}_{2}(\mathrm{Ph})$ & 2163 & 2064 & 1.143 & 0.072031 & 0.145759 & -2404.80270593 \\
\hline $\mathrm{BH}_{3}$ & 2152 & 2053 & 1.145 & 0.012388 & 0.068313 & -1975.07869903 \\
\hline $\mathrm{Al}\left(\mathrm{CH}_{3}\right)_{3}$ & 2156 & 2057 & 1.144 & 0.075190 & 0.152234 & -2310.68185436 \\
\hline $\mathrm{BF}_{3}$ & 2165 & 2066 & 1.143 & -0.008842 & 0.053616 & -2273.06737751 \\
\hline
\end{tabular}

Table S10. Formation energies and enthalpies of Lewis acid adducts of $\left[\mathrm{Ni}(\mathrm{CO})_{3}-\mathrm{F}\right]^{-}$.<smiles>CCO[N+](=O)[O-]</smiles>

\begin{tabular}{|l|l|l|}
\hline Lewis acid & $\begin{array}{l}\Delta \mathbf{G} \\
\text { (kcal/mol) }\end{array}$ & $\begin{array}{l}\Delta \mathbf{H} \\
\text { (kcal/mol) }\end{array}$ \\
\hline $\mathrm{B}\left(\mathrm{OCH}_{3}\right)_{3}$ & 5.5 & -6.0 \\
\hline $\mathrm{BPin}\left(\mathrm{CH}_{3}\right)$ & 7.0 & -5.1 \\
\hline $\mathrm{B}\left(\mathrm{CH}_{3}\right)_{3}$ & -2.5 & -14.6 \\
\hline $\mathrm{B}\left(\mathrm{CH}_{3}\right)_{2}(\mathrm{OPh})$ & -1.6 & -13.2 \\
\hline $\mathrm{BF}_{2}(\mathrm{Ph})$ & -19.8 & -29.5 \\
\hline $\mathrm{BH}_{3}$ & -23.8 & -33.6 \\
\hline $\mathrm{Al}_{3}\left(\mathrm{CH}_{3}\right)_{3}$ & -27.3 & -37.2 \\
\hline $\mathrm{BF}_{3}$ & -32.5 & -43.0 \\
\hline
\end{tabular}


Table S11. Parameters of $\left[\mathrm{Ni}(\mathrm{CO})_{3}-\mathrm{Cl}\right]^{-}$and adducts.<smiles>O=[N+]([O-])[O-]</smiles>

\begin{tabular}{|c|c|c|c|c|c|c|}
\hline Lewis acid & $\begin{array}{l}\mathrm{vCO}_{\mathrm{a} 1} \\
\left(\mathrm{~cm}^{-1}\right)\end{array}$ & $\begin{array}{l}\mathrm{vCO}_{\mathrm{a} 1} \\
\left(\mathrm{~cm}^{-1}\right) \\
\text { calibrated }\end{array}$ & $\begin{array}{l}\text { Ave C-O } \\
\text { (Å) }\end{array}$ & $\mathrm{G}_{\text {corr }}($ Hartree) & $\mathrm{H}_{\text {corr }}$ (Hartree) & $\begin{array}{l}\mathrm{SCF}\left(\mathrm{CH}_{2} \mathrm{Cl}_{2}\right) \\
\text { (Hartree) }\end{array}$ \\
\hline- & 2125 & 2027 & 1.148 & -0.014070 & 0.035194 & -2308.83940920 \\
\hline $\mathrm{B}\left(\mathrm{OCH}_{3}\right)_{3}$ & - & & & & & \\
\hline $\mathrm{Bpin}\left(\mathrm{CH}_{3}\right)$ & - & & & & & \\
\hline $\mathrm{B}\left(\mathrm{CH}_{3}\right)_{3}$ & 2144 & 2045 & 1.145 & 0.090197 & 0.158032 & -2453.45073627 \\
\hline $\mathrm{B}\left(\mathrm{CH}_{3}\right)_{2}(\mathrm{OPh})$ & 2150 & 2051 & 1.144 & 0.142009 & 0.220771 & -2720.44774398 \\
\hline $\mathrm{BF}_{2}(\mathrm{Ph})$ & 2153 & 2054 & 1.144 & 0.070750 & 0.145169 & -2765.18238624 \\
\hline $\mathrm{BH}_{3}$ & 2147 & 2048 & 1.145 & 0.011224 & 0.068080 & -2335.47431743 \\
\hline $\mathrm{Al}\left(\mathrm{CH}_{3}\right)_{3}$ & 2153 & 2054 & 1.144 & 0.078195 & 0.152244 & -2671.07040047 \\
\hline $\mathrm{BF}_{3}$ & 2156 & 2057 & 1.144 & -0.010212 & 0.052869 & -2633.44204511 \\
\hline
\end{tabular}

Table S12. Formation energies and enthalpies of Lewis acid adducts of $\left[\mathrm{Ni}(\mathrm{CO})_{3}-\mathrm{Cl}\right]^{-}$.

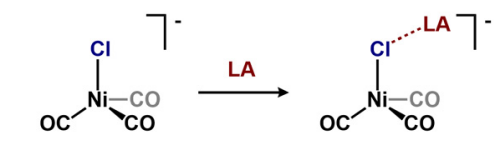

\begin{tabular}{|l|l|l|}
\hline Lewis acid & $\begin{array}{l}\Delta \mathbf{G} \\
\text { (kcal/mol) }\end{array}$ & $\begin{array}{l}\mathbf{\Delta H} \\
\text { (kcal/mol) }\end{array}$ \\
\hline $\mathrm{B}\left(\mathrm{OCH}_{3}\right)_{3}$ & & \\
\hline $\mathrm{Bpin}\left(\mathrm{CH}_{3}\right)$ & & \\
\hline $\mathrm{B}\left(\mathrm{CH}_{3}\right)_{3}$ & 13.4 & 0.7 \\
\hline $\mathrm{B}\left(\mathrm{CH}_{3}\right)_{2}(\mathrm{OPh})$ & 19.8 & 7.7 \\
\hline $\mathrm{BF}_{2}(\mathrm{Ph})$ & 4.9 & -4.9 \\
\hline $\mathrm{BH}_{3}$ & -9.0 & -18.7 \\
\hline $\mathrm{Al}_{3}\left(\mathrm{CH}_{3}\right)_{3}$ & -5.5 & -17.7 \\
\hline $\mathrm{BF}_{3}$ & -4.7 & -15.3 \\
\hline
\end{tabular}


Table S13. Parameters of $\left.\mathrm{Ni}(\mathrm{CO})_{3}-\mathrm{Br}\right]^{-}$and adducts.

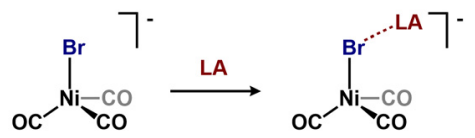

\begin{tabular}{|c|c|c|c|c|c|c|}
\hline Lewis acid & $\begin{array}{l}\mathrm{vCO}_{\mathrm{a} 1} \\
\left(\mathrm{~cm}^{-1}\right)\end{array}$ & $\begin{array}{l}v \mathrm{CO}_{\mathrm{a} 1} \\
\left(\mathrm{~cm}^{-1}\right) \\
\text { calibrated }\end{array}$ & $\begin{array}{l}\text { Ave C-O } \\
\text { (Å) }\end{array}$ & $\mathrm{G}_{\text {corr }}($ Hartree $)$ & $\mathrm{H}_{\text {corr }}$ (Hartree) & $\begin{array}{l}\mathrm{SCF}\left(\mathrm{CH}_{2} \mathrm{Cl}_{2}\right) \\
\text { (Hartree) }\end{array}$ \\
\hline- & 2126 & 2028 & 1.147 & -0.015198 & 0.035184 & -4422.92265208 \\
\hline $\mathrm{B}\left(\mathrm{OCH}_{3}\right)_{3}$ & - & & & & & \\
\hline $\mathrm{Bpin}\left(\mathrm{CH}_{3}\right)$ & - & & & & & \\
\hline $\mathrm{B}\left(\mathrm{CH}_{3}\right)_{3}$ & 2141 & 2042 & 1.145 & 0.088311 & 0.158007 & -4567.53080457 \\
\hline $\mathrm{B}\left(\mathrm{CH}_{3}\right)_{2}(\mathrm{OPh})$ & 2146 & 2047 & 1.144 & 0.139555 & 0.220672 & -4834.52588143 \\
\hline $\mathrm{BF}_{2}(\mathrm{Ph})$ & 2151 & 2052 & 1.145 & 0.085966 & 0.158525 & -2380.77108945 \\
\hline $\mathrm{BH}_{3}$ & 2144 & 2046 & 1.145 & 0.009367 & 0.067858 & -4449.55374806 \\
\hline $\mathrm{Al}\left(\mathrm{CH}_{3}\right)_{3}$ & 2151 & 2052 & 1.144 & 0.076997 & 0.152228 & -4785.14852114 \\
\hline $\mathrm{BF}_{3}$ & 2152 & 2053 & 1.144 & -0.012279 & 0.052709 & -4747.51701781 \\
\hline
\end{tabular}

Table S14. Formation energies and enthalpies of Lewis acid adducts of $\left[\mathrm{Ni}(\mathrm{CO})_{3}-\mathrm{Br}\right]^{-}$.

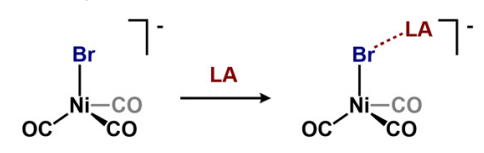

\begin{tabular}{|l|l|l|}
\hline Lewis acid & $\begin{array}{l}\Delta \mathbf{G} \\
\text { (kcal/mol) }\end{array}$ & $\begin{array}{l}\Delta \mathbf{H} \\
\text { (kcal/mol) }\end{array}$ \\
\hline $\mathrm{B}\left(\mathrm{OCH}_{3}\right)_{3}$ & & \\
\hline $\mathrm{Bpin}\left(\mathrm{CH}_{3}\right)$ & & \\
\hline $\mathrm{B}\left(\mathrm{CH}_{3}\right)_{3}$ & 14.9 & 2.7 \\
\hline $\mathrm{B}\left(\mathrm{CH}_{3}\right)_{2}(\mathrm{OPh})$ & 22.2 & 10.8 \\
\hline $\mathrm{BF}_{2}(\mathrm{Ph})$ & -28.9 & -40.3 \\
\hline $\mathrm{BH}_{3}$ & -7.1 & -16.5 \\
\hline $\mathrm{Al}_{3}\left(\mathrm{CH}_{3}\right)_{3}$ & -2.3 & -14.5 \\
\hline $\mathrm{BF}_{3}$ & -0.1 & -10.2 \\
\hline
\end{tabular}


Table S15. Parameters of $\left[\mathrm{Ni}(\mathrm{CO})_{3}-\mathrm{OH}\right]^{-}$and adducts.

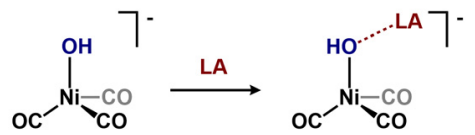

\begin{tabular}{|c|c|c|c|c|c|c|}
\hline Lewis acid & $\begin{array}{l}\mathrm{vCO}_{\mathrm{a} 1} \\
\left(\mathrm{~cm}^{-1}\right)\end{array}$ & $\begin{array}{l}v \mathrm{CO}_{\mathrm{a} 1} \\
\left(\mathrm{~cm}^{-1}\right) \\
\text { calibrated }\end{array}$ & $\begin{array}{l}\text { Ave C-O } \\
\text { (Å) }\end{array}$ & $\mathbf{G}_{\text {corr }}($ Hartree $)$ & $\mathrm{H}_{\text {corr }}($ Hartree $)$ & $\begin{array}{l}\mathrm{SCF}\left(\mathrm{CH}_{2} \mathrm{Cl}_{2}\right) \\
\text { (Hartree) }\end{array}$ \\
\hline- & 2101 & 2004 & 1.151 & -0.003139 & 0.046845 & -1924.36991939 \\
\hline $\mathrm{B}\left(\mathrm{OCH}_{3}\right)_{3}$ & 2145 & 2046 & 1.146 & 0.115993 & 0.191717 & -2294.79012005 \\
\hline $\mathrm{Bpin}\left(\mathrm{CH}_{3}\right)$ & 2144 & 2046 & 1.146 & 0.201697 & 0.279232 & -2375.61053530 \\
\hline $\mathrm{B}\left(\mathrm{CH}_{3}\right)_{3}$ & 2139 & 2040 & 1.146 & 0.104592 & 0.170562 & -2069.02133543 \\
\hline $\mathrm{B}\left(\mathrm{CH}_{3}\right)_{2}(\mathrm{OPh})$ & 2147 & 2048 & 1.145 & 0.154240 & 0.233734 & -2336.02925328 \\
\hline $\mathrm{BF}_{2}(\mathrm{Ph})$ & 2151 & 2052 & 1.145 & 0.085966 & 0.158525 & -2380.77108945 \\
\hline $\mathrm{BH}_{3}$ & 2139 & 2040 & 1.146 & 0.026229 & 0.081062 & -1951.04864333 \\
\hline $\mathrm{Al}\left(\mathrm{CH}_{3}\right)_{3}$ & 2143 & 2045 & 1.146 & 0.090280 & 0.164360 & -2286.64101962 \\
\hline $\mathrm{BF}_{3}$ & 2153 & 2053 & 1.145 & 0.004721 & 0.066366 & -2249.03706283 \\
\hline
\end{tabular}

Table S16. Formation energies and enthalpies of Lewis acid adducts of $\left[\mathrm{Ni}(\mathrm{CO})_{3}-\mathrm{OH}\right]^{-}$.

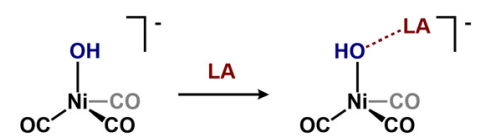

\begin{tabular}{|l|l|l|}
\hline Lewis acid & $\begin{array}{l}\Delta \mathbf{G} \\
\text { (kcal/mol) }\end{array}$ & $\begin{array}{l}\Delta \mathbf{H} \\
\text { (kcal/mol) }\end{array}$ \\
\hline- & & \\
\hline $\mathrm{B}\left(\mathrm{OCH}_{3}\right)_{3}$ & -1.8 & -15.2 \\
\hline $\mathrm{Bpin}\left(\mathrm{CH}_{3}\right)$ & -0.7 & -14.0 \\
\hline $\mathrm{B}\left(\mathrm{CH}_{3}\right)_{3}$ & -9.5 & -23.9 \\
\hline $\mathrm{B}\left(\mathrm{CH}_{3}\right)_{2}(\mathrm{OPh})$ & -11.3 & -23.5 \\
\hline $\mathrm{BF} 2(\mathrm{Ph})$ & -28.9 & -40.3 \\
\hline $\mathrm{BH}_{3}$ & -33.9 & -45.4 \\
\hline $\mathrm{Al}_{(}\left(\mathrm{CH}_{3}\right)_{3}$ & -29.9 & -42.6 \\
\hline $\mathrm{BF}_{3}$ & -42.7 & -54.6 \\
\hline
\end{tabular}


Table S17. Parameters of $\left[\mathrm{Ni}(\mathrm{CO})_{3}-\mathrm{NH}_{2}\right]^{-}$and adducts.<smiles></smiles>

\begin{tabular}{|c|c|c|c|c|c|c|}
\hline Lewis acid & $\begin{array}{l}\mathrm{vCO}_{\mathrm{a} 1} \\
\left(\mathrm{~cm}^{-1}\right)\end{array}$ & $\begin{array}{l}\mathrm{vCO}_{\mathrm{a} 1} \\
\left(\mathrm{~cm}^{-1}\right) \\
\text { calibrated }\end{array}$ & $\begin{array}{l}\text { Ave C-O } \\
\text { (Å) }\end{array}$ & $G_{\text {corr }}($ Hartree $)$ & $\mathrm{H}_{\text {corr }}$ (Hartree) & $\begin{array}{l}\mathrm{SCF}\left(\mathrm{CH}_{2} \mathrm{Cl}_{2}\right) \\
\text { (Hartree) }\end{array}$ \\
\hline- & 2086 & 1990 & 1.153 & 0.009525 & 0.059164 & -1904.48182378 \\
\hline $\mathrm{B}\left(\mathrm{OCH}_{3}\right)_{3}$ & 2132 & 2034 & 1.147 & 0.130839 & 0.204924 & -2274.90895354 \\
\hline $\mathrm{Bpin}\left(\mathrm{CH}_{3}\right)$ & 2133 & 2034 & 1.147 & 0.215999 & 0.292652 & -2355.73298942 \\
\hline $\mathrm{B}\left(\mathrm{CH}_{3}\right)_{3}$ & 2124 & 2026 & 1.148 & 0.118404 & 0.184093 & -2049.14787989 \\
\hline $\mathrm{B}\left(\mathrm{CH}_{3}\right)_{2}(\mathrm{OPh})$ & 2132 & 2034 & 1.147 & 0.172710 & 0.246181 & -2316.15285169 \\
\hline $\mathrm{BF}_{2}(\mathrm{Ph})$ & 2136 & 2038 & 1.147 & 0.100876 & 0.171880 & -2360.89487558 \\
\hline $\mathrm{BH}_{3}$ & 2124 & 2026 & 1.148 & 0.040738 & 0.094514 & -1931.17960404 \\
\hline $\mathrm{Al}\left(\mathrm{CH}_{3}\right)_{3}$ & 2130 & 2032 & 1.147 & 0.105883 & 0.177429 & -2266.75680281 \\
\hline $\mathrm{BF}_{3}$ & 2139 & 2040 & 1.146 & 0.019359 & 0.079748 & -2229.16182223 \\
\hline
\end{tabular}

Table S18. Formation energies and enthalpies of Lewis acid adducts of $\left[\mathrm{Ni}(\mathrm{CO})_{3}-\mathrm{NH}_{2}\right]^{-}$.

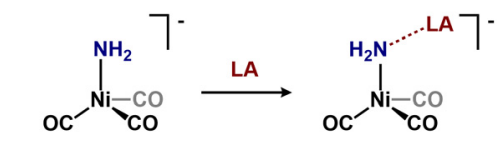

\begin{tabular}{|l|l|l|}
\hline Lewis acid & $\begin{array}{l}\Delta \mathbf{G} \\
\text { (kcal/mol) }\end{array}$ & $\begin{array}{l}\Delta \mathbf{H} \\
\text { (kcal/mol) }\end{array}$ \\
\hline $\mathrm{B}\left(\mathrm{OCH}_{3}\right)_{3}$ & -4.8 & -18.9 \\
\hline $\mathrm{BPin}\left(\mathrm{CH}_{3}\right)$ & -6.3 & -19.9 \\
\hline $\mathrm{B}\left(\mathrm{CH}_{3}\right)_{3}$ & -18.0 & -32.3 \\
\hline $\mathrm{B}\left(\mathrm{CH}_{3}\right)_{2}(\mathrm{OPh})$ & -15.0 & -30.7 \\
\hline $\mathrm{BF}_{2}(\mathrm{Ph})$ & -34.9 & -47.1 \\
\hline $\mathrm{BH}_{3}$ & -44.7 & -56.6 \\
\hline $\mathrm{Al}_{3}\left(\mathrm{CH}_{3}\right)_{3}$ & -30.5 & -44.5 \\
\hline $\mathrm{BF}_{3}$ & -49.5 & -61.9 \\
\hline
\end{tabular}


Table S19. Parameters of $\left[\mathrm{Ni}(\mathrm{CO})_{3}-\mathrm{PHPh}\right]^{-}$and adducts.

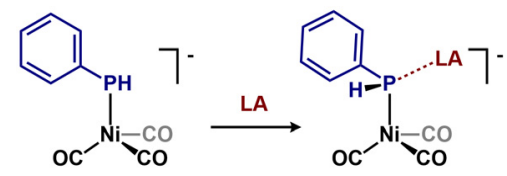

\begin{tabular}{|l|l|l|l|l|l|l|}
\hline Lewis acid & $\begin{array}{l}\mathbf{v} \mathbf{C O}_{\mathbf{a}} \\
\left(\mathbf{c m}^{-1}\right)\end{array}$ & $\begin{array}{l}\mathbf{v C O} \\
\left(\mathbf{c m}^{-1} \mathbf{)}\right. \\
\text { calibrated }\end{array}$ & $\begin{array}{l}\text { Ave } \mathbf{C}-\mathbf{O} \\
(\AA)\end{array}$ & $\mathbf{G}_{\text {corr }}($ Hartree) & $\mathbf{H}_{\text {corr }}($ Hartree $)$ & $\begin{array}{l}\mathbf{S C F}\left(\mathbf{C H}_{2} \mathbf{C l}_{2}\right) \\
(\text { Hartree })\end{array}$ \\
\hline- & 2096 & 1999 & 1.150 & 0.076934 & 0.140248 & -2422.18566015 \\
\hline $\mathrm{B}\left(\mathrm{OCH}_{3}\right)_{3}$ & - & & & & & \\
\hline $\mathrm{Bpin}\left(\mathrm{CH}_{3}\right)$ & 2128 & 2030 & 1.146 & 0.281699 & 0.372146 & -2873.39348897 \\
\hline $\mathrm{B}\left(\mathrm{CH}_{3}\right)_{3}$ & 2124 & 2026 & 1.146 & 0.185063 & 0.263900 & -2566.82180181 \\
\hline $\mathrm{B}\left(\mathrm{CH}_{3}\right)_{2}(\mathrm{OPh})$ & 2131 & 2033 & 1.146 & 0.236190 & 0.326681 & -2833.81873814 \\
\hline $\mathrm{BF}_{2}(\mathrm{Ph})$ & 2132 & 2034 & 1.145 & 0.164843 & 0.251035 & -2878.55206039 \\
\hline $\mathrm{BH}_{3}$ & 2123 & 2025 & 1.147 & 0.105518 & 0.173619 & -2448.85838492 \\
\hline $\mathrm{Al}_{(}\left(\mathrm{CH}_{3}\right)_{3}$ & 2128 & 2030 & 1.146 & 0.171021 & 0.257998 & -2784.42716859 \\
\hline $\mathrm{BF}_{3}$ & 2135 & 2037 & 1.145 & 0.083196 & 0.158799 & -2746.81291775 \\
\hline
\end{tabular}

Table S20. Formation energies and enthalpies of Lewis acid adducts of $\left[\mathrm{Ni}(\mathrm{CO})_{3}-\mathrm{PHPh}\right]^{-}$.

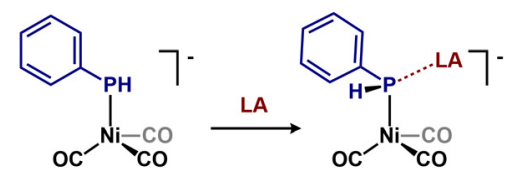

\begin{tabular}{|l|l|l|}
\hline Lewis acid & $\begin{array}{l}\mathbf{\Delta G} \\
(\mathbf{k c a l} / \mathrm{mol})\end{array}$ & $\begin{array}{l}\mathbf{\Delta H} \\
(\mathbf{k c a l} / \mathbf{m o l})\end{array}$ \\
\hline $\mathrm{B}\left(\mathrm{OCH}_{3}\right)_{3}$ & & \\
\hline $\mathrm{Bpin}\left(\mathrm{CH}_{3}\right)$ & 19.8 & 6.2 \\
\hline $\mathrm{B}\left(\mathrm{CH}_{3}\right)_{3}$ & 0.3 & -14.3 \\
\hline $\mathrm{B}\left(\mathrm{CH}_{3}\right)_{2}(\mathrm{OPh})$ & 6.3 & -7.3 \\
\hline $\mathrm{BF}(\mathrm{Ph})$ & -7.8 & -19.0 \\
\hline $\mathrm{BH}_{3}$ & -30.7 & -42.1 \\
\hline $\mathrm{Al}\left(\mathrm{CH}_{3}\right)_{3}$ & -10.9 & -23.9 \\
\hline $\mathrm{BF}_{3}$ & -18.7 & -30.2 \\
\hline
\end{tabular}


Table S21. Parameters of [ $\left.\mathrm{Ni}(\mathrm{CO})_{3}-\mathrm{SPh}\right]^{-}$and adducts.

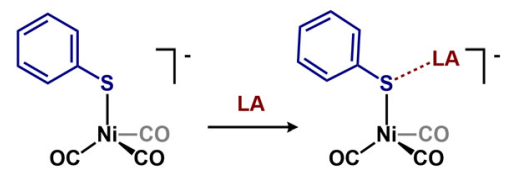

\begin{tabular}{|l|l|l|l|l|l|l|}
\hline Lewis acid & $\begin{array}{l}\mathbf{v} \mathbf{C O}_{\mathbf{a}} \\
\left(\mathbf{c m}^{-1}\right)\end{array}$ & $\begin{array}{l}\mathbf{v C O} \\
\left(\mathbf{c m}^{-1} \mathbf{)}\right. \\
\text { calibrated }\end{array}$ & $\begin{array}{l}\text { Ave } \mathbf{C}-\mathbf{O} \\
(\AA)\end{array}$ & $\mathbf{G}_{\text {corr }}($ Hartree) & $\mathbf{H}_{\text {corr }}($ Hartree $)$ & $\begin{array}{l}\mathbf{S C F}\left(\mathbf{C H}_{2} \mathbf{C l}_{2}\right) \\
(\text { Hartree })\end{array}$ \\
\hline- & 2120 & 2023 & 1.148 & 0.068998 & 0.131318 & -2478.45106069 \\
\hline $\mathrm{B}\left(\mathrm{OCH}_{3}\right)_{3}$ & 2135 & 2037 & 1.145 & 0.186417 & 0.275297 & -2848.82695740 \\
\hline $\mathrm{Bpin}\left(\mathrm{CH}_{3}\right)$ & - & & & & & \\
\hline $\mathrm{B}\left(\mathrm{CH}_{3}\right)_{3}$ & 2134 & 2035 & 1.145 & 0.174315 & 0.254627 & -2623.07044783 \\
\hline $\mathrm{B}\left(\mathrm{CH}_{3}\right)_{2}(\mathrm{OPh})$ & 2142 & 2043 & 1.144 & 0.228655 & 0.317529 & -2890.06982693 \\
\hline $\mathrm{BF}_{2}(\mathrm{Ph})$ & 2142 & 2043 & 1.144 & 0.156682 & 0.241784 & -2934.80444791 \\
\hline $\mathrm{BH}_{3}$ & 2137 & 2039 & 1.145 & 0.097124 & 0.164580 & -2505.10561239 \\
\hline $\mathrm{Al}_{(}\left(\mathrm{CH}_{3}\right)_{3}$ & 2140 & 2041 & 1.145 & 0.162663 & 0.248896 & -2840.68519978 \\
\hline $\mathrm{BF}_{3}$ & 2145 & 2047 & 1.144 & 0.074978 & 0.149504 & -2803.06529217 \\
\hline
\end{tabular}

Table S22. Formation energies and enthalpies of Lewis acid adducts of $\left[\mathrm{Ni}(\mathrm{CO})_{3}-\mathrm{SPh}\right]^{-}$.

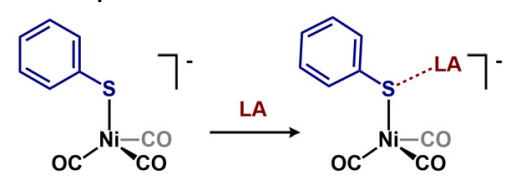

\begin{tabular}{|l|l|l|}
\hline Lewis acid & $\begin{array}{l}\mathbf{\Delta G} \\
(\mathbf{k c a l} / \mathrm{mol})\end{array}$ & $\begin{array}{l}\mathbf{\Delta H} \\
(\mathbf{k c a l} / \mathbf{m o l})\end{array}$ \\
\hline $\mathrm{B}\left(\mathrm{OCH}_{3}\right)_{3}$ & 24.9 & 12.1 \\
\hline $\mathrm{Bpin}\left(\mathrm{CH}_{3}\right)$ & & \\
\hline $\mathrm{B}\left(\mathrm{CH}_{3}\right)_{3}$ & 9.0 & -4.0 \\
\hline $\mathrm{B}\left(\mathrm{CH}_{3}\right)_{2}(\mathrm{OPh})$ & 15.6 & 1.5 \\
\hline $\mathrm{BF}(\mathrm{Ph})$ & 0.2 & -11.1 \\
\hline $\mathrm{BH}_{3}$ & -19.6 & -30.8 \\
\hline $\mathrm{Al}\left(\mathrm{CH}_{3}\right)_{3}$ & -6.6 & -19.3 \\
\hline $\mathrm{BF}_{3}$ & -10.7 & -22.2 \\
\hline
\end{tabular}


Table S23. Parameters of $\left[\mathrm{Ni}(\mathrm{CO})_{3}\right]$-pyrazine and adducts.

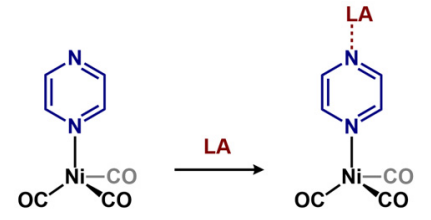

\begin{tabular}{|c|c|c|c|c|c|c|}
\hline Lewis acid & $\begin{array}{l}\mathrm{vCO}_{\mathrm{a} 1} \\
\left(\mathrm{~cm}^{-1}\right)\end{array}$ & $\begin{array}{l}\mathrm{CO}_{\mathrm{a} 1} \\
\left(\mathrm{~cm}^{-1}\right) \\
\text { calibrated }\end{array}$ & $\begin{array}{l}\text { Ave C-O } \\
\text { (A) }\end{array}$ & $\mathbf{G}_{\text {corr }}$ (Hartree) & $\mathbf{H}_{\text {corr }}$ (Hartree) & $\begin{array}{l}\mathrm{SCF}\left(\mathrm{CH}_{2} \mathrm{Cl}_{2}\right) \\
\text { (Hartree) }\end{array}$ \\
\hline- & 2175 & 2075 & 1.140 & 0.057633 & 0.117221 & -2112.75755485 \\
\hline $\mathrm{B}\left(\mathrm{OCH}_{3}\right)_{3}$ & - & & & & & \\
\hline $\mathrm{Bpin}\left(\mathrm{CH}_{3}\right)$ & - & & & & & \\
\hline $\mathrm{B}\left(\mathrm{CH}_{3}\right)_{3}$ & 2178 & 2078 & 1.139 & 0.167125 & 0.241671 & -2257.38552287 \\
\hline $\mathrm{B}\left(\mathrm{CH}_{3}\right)_{2}(\mathrm{OPh})$ & 2178 & 2078 & 1.139 & 0.217871 & 0.304281 & -2524.38285210 \\
\hline $\mathrm{BF}_{2}(\mathrm{Ph})$ & 2180 & 2080 & 1.139 & 0.144208 & 0.228543 & -2569.11325781 \\
\hline $\mathrm{BH}_{3}$ & 2179 & 2078 & 1.139 & 0.086899 & 0.151580 & -2139.41403655 \\
\hline $\mathrm{Al}\left(\mathrm{CH}_{3}\right)_{3}$ & 2179 & 2079 & 1.139 & 0.155786 & 0.234266 & -2474.98896577 \\
\hline $\mathrm{BF}_{3}$ & 2182 & 2081 & 1.138 & 0.064901 & 0.136137 & -2437.37252960 \\
\hline
\end{tabular}

Table S24. Formation energies and enthalpies of Lewis acid adducts of $\left[\mathrm{Ni}(\mathrm{CO})_{3}\right]$-pyrazine.

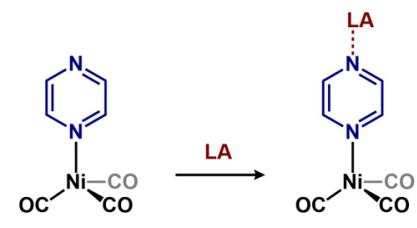

\begin{tabular}{|l|l|l|}
\hline Lewis acid & $\begin{array}{l}\Delta \mathbf{G} \\
(\mathbf{k c a l} / \mathrm{mol})\end{array}$ & $\begin{array}{l}\Delta \mathbf{H} \\
(\mathbf{k c a l} / \mathrm{mol})\end{array}$ \\
\hline $\mathrm{B}\left(\mathrm{OCH}_{3}\right)_{3}$ & & \\
\hline $\mathrm{Bpin}\left(\mathrm{CH}_{3}\right)$ & & \\
\hline $\mathrm{B}\left(\mathrm{CH}_{3}\right)_{3}$ & 6.3 & -8.7 \\
\hline $\mathrm{B}\left(\mathrm{CH}_{3}\right)_{2}(\mathrm{OPh})$ & 11.8 & -2.0 \\
\hline $\mathrm{BF}_{2}(\mathrm{Ph})$ & -1.9 & -12.0 \\
\hline $\mathrm{BH}_{3}$ & -20.1 & -31.3 \\
\hline $\mathrm{Al}\left(\mathrm{CH}_{3}\right)_{3}$ & -2.0 & -18.0 \\
\hline $\mathrm{BF}_{3}$ & -10.3 & -22.2 \\
\hline
\end{tabular}


Table S25. Parameters of $\left[\mathrm{Ni}(\mathrm{CO})_{3}\right]$-pyrimidine and adducts.

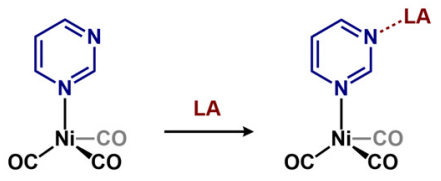

\begin{tabular}{|l|l|l|l|l|l|l|}
\hline Lewis acid & $\begin{array}{l}\mathbf{v} \mathbf{C O}_{a 1} \\
\left(\mathbf{c m}^{-1}\right)\end{array}$ & $\begin{array}{l}\mathbf{v C O} \\
\left(\mathbf{c m}^{-1}\right) \\
\text { calibrated }\end{array}$ & $\begin{array}{l}\text { Ave C-O } \\
(\AA)\end{array}$ & $\mathbf{G}_{\text {corr }}($ Hartree) & $\mathbf{H}_{\text {corr }}($ Hartree) & $\begin{array}{l}\mathbf{S C F}\left(\mathbf{C H}_{2} \mathbf{C l}_{2}\right) \\
(\text { Hartree })\end{array}$ \\
\hline- & 2174 & 2074 & 1.140 & 0.057439 & 0.117424 & -2112.76545822 \\
\hline $\mathrm{B}\left(\mathrm{OCH}_{3}\right)_{3}$ & - & & & & & \\
\hline $\mathrm{Bpin}\left(\mathrm{CH}_{3}\right)$ & - & & & & & \\
\hline $\mathrm{B}\left(\mathrm{CH}_{3}\right)_{3}$ & 2179 & 2079 & 1.139 & 0.165509 & 0.241800 & -2257.39205278 \\
\hline $\mathrm{B}\left(\mathrm{CH}_{3}\right)_{2}(\mathrm{OPh})$ & 2180 & 2079 & 1.139 & 0.217419 & 0.304424 & -2524.38948973 \\
\hline $\mathrm{BF}{ }_{2}(\mathrm{Ph})$ & 2181 & 2081 & 1.139 & 0.146367 & 0.228703 & -2569.12045852 \\
\hline $\mathrm{BH}_{3}$ & 2180 & 2080 & 1.139 & 0.085087 & 0.151766 & -2139.42054700 \\
\hline $\left.\mathrm{Al}_{(\mathrm{CH}}\right)_{3}$ & 2180 & 2080 & 1.139 & 0.152567 & 0.235412 & -2474.99673767 \\
\hline $\mathrm{BF}_{3}$ & 2183 & 2082 & 1.139 & 0.063996 & 0.136299 & -2437.37990962 \\
\hline
\end{tabular}

Table S26. Formation energies and enthalpies of Lewis acid adducts of $\left[\mathrm{Ni}(\mathrm{CO})_{3}\right]$-pyrimidine.

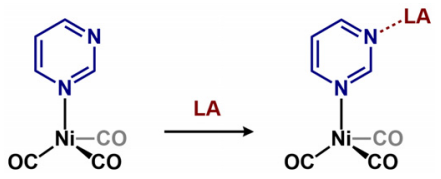

\begin{tabular}{|l|l|l|}
\hline Lewis acid & $\begin{array}{l}\Delta \mathbf{G} \\
\text { (kcal/mol) }\end{array}$ & $\begin{array}{l}\mathbf{\Delta} \\
\text { (kcal/mol) }\end{array}$ \\
\hline $\mathrm{B}\left(\mathrm{OCH}_{3}\right)_{3}$ & & \\
\hline $\mathrm{Bpin}\left(\mathrm{CH}_{3}\right)$ & & \\
\hline $\mathrm{B}\left(\mathrm{CH}_{3}\right)_{3}$ & 6.2 & -7.9 \\
\hline $\mathrm{B}\left(\mathrm{CH}_{3}\right)_{2}(\mathrm{OPh})$ & 12.5 & -1.3 \\
\hline $\mathrm{BF}(\mathrm{Ph})$ & 0.0 & -11.6 \\
\hline $\mathrm{BH}_{3}$ & -20.2 & -30.5 \\
\hline $\mathrm{Al}_{3}\left(\mathrm{CH}_{3}\right)_{3}$ & -3.9 & -17.3 \\
\hline $\mathrm{BF}_{3}$ & -10.4 & -21.9 \\
\hline
\end{tabular}


Table S27. Parameters of $\left[\mathrm{Ni}(\mathrm{CO})_{3}\right]$-pyridazine and adducts.

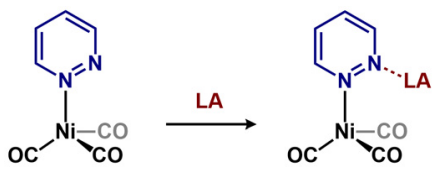

\begin{tabular}{|c|c|c|c|c|c|c|}
\hline Lewis acid & $\begin{array}{l}\mathrm{vCO}_{\mathrm{a}} \\
\left(\mathrm{cm}^{-1}\right)\end{array}$ & $\begin{array}{l}\mathrm{vCO}_{\mathrm{a} 1} \\
\left(\mathrm{~cm}^{-1}\right) \\
\text { calibrated }\end{array}$ & $\begin{array}{l}\text { Ave C-O } \\
\text { (Å) }\end{array}$ & $\mathbf{G}_{\text {corr }}$ (Hartree) & $\mathrm{H}_{\text {corr }}($ Hartree) & $\begin{array}{l}\mathrm{SCF}\left(\mathrm{CH}_{2} \mathrm{Cl}_{2}\right) \\
\text { (Hartree) }\end{array}$ \\
\hline - & 2173 & 2073 & 1.140 & 0.057537 & 0.116682 & \\
\hline $\mathrm{BF}_{3}$ & 2185 & 2085 & 1.138 & 0.066519 & 0.135550 & -2437.34140870 \\
\hline
\end{tabular}

Table S28. Formation energies and enthalpies of Lewis acid adducts of $\left[\mathrm{Ni}(\mathrm{CO})_{3}\right]$-pyridazine.

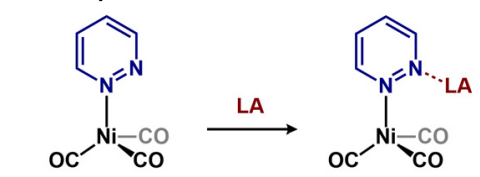

\begin{tabular}{|l|l|l|}
\hline Lewis acid & $\begin{array}{l}\mathbf{\Delta G} \\
\text { (kcal/mol) }\end{array}$ & $\begin{array}{l}\mathbf{\Delta H} \\
\text { (kcal/mol) }\end{array}$ \\
\hline $\mathrm{BF}_{3}$ & -4.9 & -17.9 \\
\hline
\end{tabular}


Table S29. Parameters of $\left[\mathrm{Ni}(\mathrm{CO})_{3}-\left(4-\mathrm{C}_{5} \mathrm{H}_{4} \mathrm{~N}\right)\right]^{-}$and adducts.

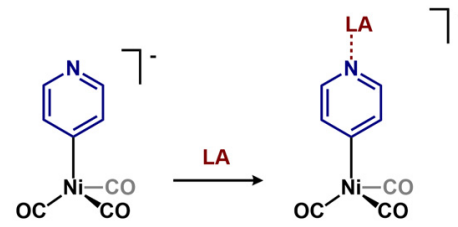

\begin{tabular}{|l|l|l|l|l|l|l|}
\hline Lewis acid & $\begin{array}{l}\mathbf{v} \mathbf{C O}_{\mathbf{a} 1} \\
\left(\mathbf{c m}^{-1}\right)\end{array}$ & $\begin{array}{l}\mathbf{v C O} \\
\left(\mathbf{c m}^{-1} \mathbf{1}\right. \\
\text { calibrated }\end{array}$ & $\begin{array}{l}\text { Ave } \mathbf{C}-\mathbf{0} \\
(\mathbf{A})\end{array}$ & $\mathbf{G}_{\text {corr }}($ Hartree) & $\mathbf{H}_{\text {corr }}($ Hartree) & $\begin{array}{l}\mathbf{S C F}\left(\mathbf{C H}_{\mathbf{2}} \mathbf{C l}_{2}\right) \\
(\text { Hartree })\end{array}$ \\
\hline- & 2098 & 2002 & 1.151 & 0.056697 & 0.115749 & -2096.21623653 \\
\hline $\mathrm{B}\left(\mathrm{OCH}_{3}\right)_{3}$ & 2110 & 2013 & 1.149 & 0.177836 & 0.260895 & -2466.61946194 \\
\hline $\mathrm{Bpin}\left(\mathrm{CH}_{3}\right)$ & 2110 & 2013 & 1.149 & 0.262219 & 0.348492 & -2547.44157229 \\
\hline $\mathrm{B}\left(\mathrm{CH}_{3}\right)_{3}$ & 2110 & 2013 & 1.149 & 0.166650 & 0.240314 & -2240.85807893 \\
\hline $\mathrm{B}\left(\mathrm{CH}_{3}\right)_{2}(\mathrm{OPh})$ & 2112 & 2015 & 1.149 & 0.217568 & 0.303198 & -2507.85944292 \\
\hline $\mathrm{BF}_{2}(\mathrm{Ph})$ & 2114 & 2017 & 1.148 & 0.146342 & 0.227449 & -2552.59440251 \\
\hline $\mathrm{BH}_{3}$ & 2109 & 2012 & 1.149 & 0.085277 & 0.150350 & -2122.88934156 \\
\hline $\mathrm{Al}_{3}\left(\mathrm{CH}_{3}\right)_{3}$ & 2112 & 2015 & 1.149 & 0.152968 & 0.233821 & -2458.46454280 \\
\hline $\mathrm{BF}_{3}$ & 2114 & 2017 & 1.148 & 0.064724 & 0.135200 & -2420.85764853 \\
\hline
\end{tabular}

Table S30. Formation energies and enthalpies of Lewis acid adducts of $\left[\mathrm{Ni}(\mathrm{CO})_{3}-\left(4-\mathrm{C}_{5} \mathrm{H}_{4} \mathrm{~N}\right)\right]^{-}$.

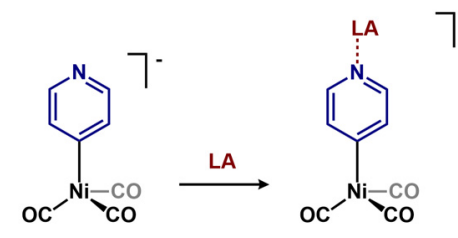

\begin{tabular}{|l|l|l|}
\hline Lewis acid & $\begin{array}{l}\mathbf{\Delta G} \\
\text { (kcal/mol) }\end{array}$ & $\begin{array}{l}\mathbf{\Delta H} \\
\text { (kcal/mol) }\end{array}$ \\
\hline $\mathrm{B}\left(\mathrm{OCH}_{3}\right)_{3}$ & 10.1 & -4.3 \\
\hline $\mathrm{Bpin}\left(\mathrm{CH}_{3}\right)$ & 9.3 & -4.2 \\
\hline $\mathrm{B}\left(\mathrm{CH}_{3}\right)_{3}$ & -2.1 & -17.3 \\
\hline $\mathrm{B}\left(\mathrm{CH}_{3}\right)_{2}(\mathrm{OPh})$ & 1.0 & -13.0 \\
\hline $\mathrm{BF}_{2}(\mathrm{Ph})$ & -14.1 & -25.8 \\
\hline $\mathrm{BH}_{3}$ & -30.9 & -41.6 \\
\hline $\mathrm{Al}\left(\mathrm{CH}_{3}\right)_{3}$ & -13.8 & -27.9 \\
\hline $\mathrm{BF}_{3}$ & -26.4 & -38.5 \\
\hline
\end{tabular}


Table S31. Parameters of $\left[\mathrm{Ni}(\mathrm{CO})_{3}-\left(3-\mathrm{C}_{5} \mathrm{H}_{4} \mathrm{~N}\right)\right]^{-}$and adducts.

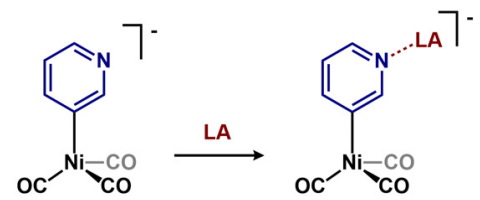

\begin{tabular}{|c|c|c|c|c|c|c|}
\hline Lewis acid & $\begin{array}{l}\mathrm{CO}_{\mathrm{a} 1} \\
\left(\mathrm{~cm}^{-1}\right)\end{array}$ & $\begin{array}{l}\mathrm{CO}_{\mathrm{a} 1} \\
\left(\mathrm{~cm}^{-1}\right) \\
\text { calibrated }\end{array}$ & $\begin{array}{l}\text { Ave C-O } \\
\text { (Å) }\end{array}$ & $\mathbf{G}_{\text {corr }}$ (Hartree) & $\mathbf{H}_{\text {corr }}$ (Hartree) & $\begin{array}{l}\mathrm{SCF}\left(\mathrm{CH}_{2} \mathrm{Cl}_{2}\right) \\
\text { (Hartree) }\end{array}$ \\
\hline- & 2096 & 2000 & 1.151 & 0.056822 & 0.115689 & -2096.21433176 \\
\hline $\mathrm{B}\left(\mathrm{OCH}_{3}\right)_{3}$ & 2109 & 2012 & 1.149 & 0.177761 & 0.260770 & -2466.61623999 \\
\hline $\operatorname{Bpin}\left(\mathrm{CH}_{3}\right)$ & 2110 & 2013 & 1.149 & 0.262694 & 0.348449 & -2547.43915776 \\
\hline $\mathrm{B}\left(\mathrm{CH}_{3}\right)_{3}$ & 2109 & 2012 & 1.149 & 0.166889 & 0.240281 & -2240.85570216 \\
\hline $\mathrm{B}\left(\mathrm{CH}_{3}\right)_{2}(\mathrm{OPh})$ & 2111 & 2014 & 1.149 & 0.217855 & 0.303161 & -2507.85740064 \\
\hline $\mathrm{BF}_{2}(\mathrm{Ph})$ & 2113 & 2016 & 1.149 & 0.146573 & 0.227385 & -2552.59167937 \\
\hline $\mathrm{BH}_{3}$ & 2109 & 2012 & 1.149 & 0.085961 & 0.150265 & -2122.88722660 \\
\hline $\mathrm{Al}\left(\mathrm{CH}_{3}\right)_{3}$ & 2111 & 2014 & 1.149 & 0.153187 & 0.233768 & -2458.46192531 \\
\hline $\mathrm{BF}_{3}$ & 2114 & 2017 & 1.149 & 0.064637 & 0.135107 & -2420.85471697 \\
\hline
\end{tabular}

Table S32. Formation energies and enthalpies of Lewis acid adducts of $\left[\mathrm{Ni}(\mathrm{CO})_{3}-\left(3-\mathrm{C}_{5} \mathrm{H}_{4} \mathrm{~N}\right)\right]^{-}$.

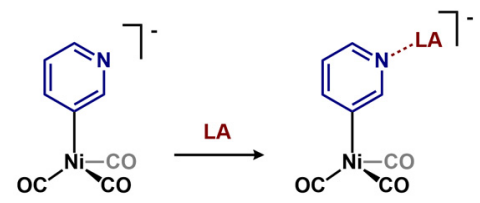

\begin{tabular}{|l|l|l|}
\hline Lewis acid & $\begin{array}{l}\Delta \mathbf{G} \\
\text { (kcal/mol) }\end{array}$ & $\begin{array}{l}\mathbf{\Delta H} \\
\text { (kcal/mol) }\end{array}$ \\
\hline $\mathrm{B}\left(\mathrm{OCH}_{3}\right)_{3}$ & 10.8 & -3.6 \\
\hline $\mathrm{Bpin}\left(\mathrm{CH}_{3}\right)$ & 9.9 & -3.9 \\
\hline $\mathrm{B}\left(\mathrm{CH}_{3}\right)_{3}$ & -1.8 & -17.0 \\
\hline $\mathrm{B}\left(\mathrm{CH}_{3}\right)_{2}(\mathrm{OPh})$ & 1.2 & -12.9 \\
\hline $\mathrm{BF}_{2}(\mathrm{Ph})$ & -13.5 & -25.3 \\
\hline $\mathrm{BH}_{3}$ & -30.4 & -41.5 \\
\hline $\mathrm{Al}\left(\mathrm{CH}_{3}\right)_{3}$ & -13.3 & -27.5 \\
\hline $\mathrm{BF}_{3}$ & -25.9 & -37.8 \\
\hline
\end{tabular}


Table S33. Parameters of $\left[\mathrm{Ni}(\mathrm{CO})_{3}-\mathrm{N}_{2} \mathrm{H}_{4}\right]$ and adducts.

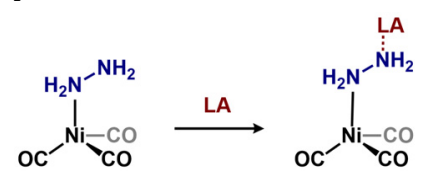

\begin{tabular}{|c|c|c|c|c|c|c|}
\hline Lewis acid & $\begin{array}{l}\mathrm{vCO} \mathrm{a}_{\mathrm{a}} \\
\left(\mathrm{cm}^{-1}\right)\end{array}$ & $\begin{array}{l}\mathrm{vCO}_{\mathrm{a} 1} \\
\left(\mathrm{~cm}^{-1}\right) \\
\text { calibrated }\end{array}$ & $\begin{array}{l}\text { Ave C-O } \\
\text { (Å) }\end{array}$ & $\mathbf{G}_{\text {corr }}$ (Hartree) & $\mathbf{H}_{\text {corr }}$ (Hartree) & $\begin{array}{l}\mathrm{SCF}\left(\mathrm{CH}_{2} \mathrm{Cl}_{2}\right) \\
\text { (Hartree) }\end{array}$ \\
\hline- & 2176 & 2076 & 1.140 & 0.040757 & 0.093606 & -1960.32574066 \\
\hline $\mathrm{B}\left(\mathrm{OCH}_{3}\right)_{3}$ & 2180 & 2080 & 1.140 & 0.159818 & 0.239508 & -2330.71493231 \\
\hline $\operatorname{Bpin}\left(\mathrm{CH}_{3}\right)$ & 2179 & 2079 & 1.140 & 0.246073 & 0.327028 & -2411.54132218 \\
\hline $\mathrm{B}\left(\mathrm{CH}_{3}\right)_{3}$ & 2182 & 2082 & 1.139 & 0.149169 & 0.218737 & -2104.96010351 \\
\hline $\mathrm{B}\left(\mathrm{CH}_{3}\right)_{2}(\mathrm{OPh})$ & 2184 & 2083 & 1.139 & 0.199684 & 0.281466 & -2371.95509958 \\
\hline $\mathrm{BF}_{2}(\mathrm{Ph})$ & 2184 & 2084 & 1.139 & 0.131382 & 0.206009 & -2416.68840622 \\
\hline $\mathrm{BH}_{3}$ & 2183 & 2083 & 1.138 & 0.071473 & 0.129229 & -1986.98731565 \\
\hline $\mathrm{Al}\left(\mathrm{CH}_{3}\right)_{3}$ & 2182 & 2081 & 1.139 & 0.135883 & 0.212064 & -2322.55912613 \\
\hline $\mathrm{BF}_{3}$ & 2191 & 2090 & 1.139 & 0.048738 & 0.113240 & -2284.94341707 \\
\hline
\end{tabular}

Table S34. Formation energies and enthalpies of Lewis acid adducts of $\left[\mathrm{Ni}(\mathrm{CO})_{3}-\mathrm{N}_{2} \mathrm{H}_{4}\right]$.

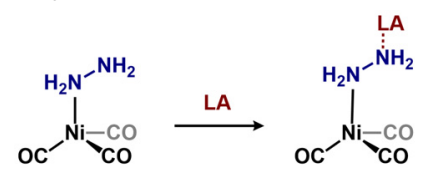

\begin{tabular}{|l|l|l|}
\hline Lewis acid & $\begin{array}{l}\Delta \mathbf{G} \\
\text { (kcal/mol) }\end{array}$ & $\begin{array}{l}\Delta \mathbf{H} \\
\text { (kcal/mol) }\end{array}$ \\
\hline $\mathrm{B}\left(\mathrm{OCH}_{3}\right)_{3}$ & 17.6 & 4.9 \\
\hline $\mathrm{Bpin}\left(\mathrm{CH}_{3}\right)$ & 15.3 & 2.3 \\
\hline $\mathrm{B}\left(\mathrm{CH}_{3}\right)_{3}$ & 1.6 & -12.3 \\
\hline $\mathrm{B}\left(\mathrm{CH}_{3}\right)_{2}(\mathrm{OPh})$ & 8.5 & -4.1 \\
\hline $\mathrm{BF}{ }_{2}(\mathrm{Ph})$ & -3.8 & -15.7 \\
\hline $\mathrm{BH}_{3}$ & -22.3 & -33.7 \\
\hline $\mathrm{Al}\left(\mathrm{CH}_{3}\right)_{3}$ & -5.2 & -18.3 \\
\hline $\mathrm{BF}_{3}$ & -11.6 & -23.5 \\
\hline
\end{tabular}


Table S35. Parameters of $\left[\mathrm{Ni}(\mathrm{CO})_{3}-\left(\mathrm{KN}-\mathrm{NO}_{2}\right)\right]^{-}$and adducts.

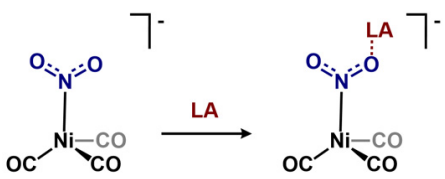

\begin{tabular}{|c|c|c|c|c|c|c|}
\hline Lewis acid & $\begin{array}{l}v \mathrm{CO}_{\mathrm{a} 1} \\
\left(\mathrm{~cm}^{-1}\right)\end{array}$ & $\begin{array}{l}\mathrm{vCO}_{\mathrm{a} 1}\left(\mathrm{~cm}^{-1}\right) \\
\text { calibrated }\end{array}$ & $\begin{array}{l}\text { Ave C-O } \\
\text { (Å) }\end{array}$ & $\begin{array}{l}\mathbf{G}_{\text {corr }} \\
\text { (Hartree) }\end{array}$ & $\begin{array}{l}\mathrm{H}_{\text {corr }} \\
\text { (Hartree) }\end{array}$ & $\begin{array}{l}\mathrm{SCF}\left(\mathrm{CH}_{2} \mathrm{Cl}_{2}\right) \\
\text { (Hartree) }\end{array}$ \\
\hline- & 2130 & 2032 & 1.147 & -0.007841 & 0.047095 & -2053.70055266 \\
\hline $\operatorname{Bpin}\left(\mathrm{CH}_{3}\right)$ & 2152 & 2053 & 1.144 & 0.196435 & 0.278763 & -2504.90704922 \\
\hline $\mathrm{B}\left(\mathrm{CH}_{3}\right)_{3}$ & 2152 & 2053 & 1.144 & 0.099230 & 0.170380 & -2198.32274330 \\
\hline $\mathrm{BH}_{3}$ & 2151 & 2052 & 1.144 & 0.021581 & 0.080654 & -2080.35222954 \\
\hline $\mathrm{Al}\left(\mathrm{CH}_{3}\right)_{3}$ & 2155 & 2056 & 1.143 & 0.086444 & 0.164336 & -2415.94020367 \\
\hline $\mathrm{BF}_{3}$ & 2159 & 2060 & 1.143 & -0.000062 & 0.065375 & -2378.32189583 \\
\hline
\end{tabular}

Table S36. Formation energies and enthalpies of Lewis acid adducts of $\left[\mathrm{Ni}(\mathrm{CO})_{3}-\left(\mathrm{KN}^{-} \mathrm{NO}_{2}\right)\right]^{-}$.

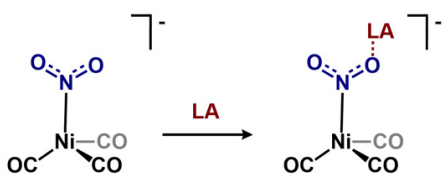

\begin{tabular}{|l|l|l|}
\hline Lewis acid & $\begin{array}{l}\Delta \mathbf{G} \\
\text { (kcal/mol) }\end{array}$ & $\begin{array}{l}\Delta \mathbf{H} \\
\text { (kcal/mol) }\end{array}$ \\
\hline $\mathrm{B}\left(\mathrm{OCH}_{3}\right)_{3}$ & & \\
\hline $\mathrm{Bpin}\left(\mathrm{CH}_{3}\right)$ & 20.4 & 6.9 \\
\hline $\mathrm{B}\left(\mathrm{CH}_{3}\right)_{3}$ & 8.4 & -5.8 \\
\hline $\mathrm{B}\left(\mathrm{CH}_{3}\right)_{2}(\mathrm{OPh})$ & 12.4 & -1.1 \\
\hline $\mathrm{BF}{ }_{2}(\mathrm{Ph})$ & -1.9 & -13.8 \\
\hline $\mathrm{BH}_{3}$ & -16.9 & -28.8 \\
\hline $\mathrm{Al}\left(\mathrm{CH}_{3}\right)_{3}$ & -9.6 & -23.0 \\
\hline $\mathrm{BF}_{3}$ & -14.0 & -26.6 \\
\hline
\end{tabular}


Table S37. Parameters of $\left[\mathrm{Ni}(\mathrm{CO})_{3}-\left(\mathrm{KO}-\mathrm{NO}_{2}\right)\right]^{-}$and adducts.

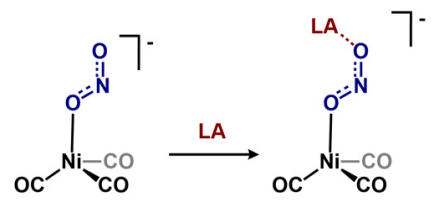

\begin{tabular}{|l|l|l|l|l|l|l|}
\hline Lewis acid & $\begin{array}{l}\mathbf{v} \mathbf{C O}_{a 1} \\
\left(\mathbf{c m}^{-1}\right)\end{array}$ & $\begin{array}{l}\mathbf{v} \mathbf{C O}_{a 1}\left(\mathbf{c m}^{-1}\right) \\
\text { calibrated }\end{array}$ & $\begin{array}{l}\text { Ave C-O } \\
(\mathbf{A})\end{array}$ & $\begin{array}{l}\mathbf{G}_{\text {corr }} \\
(\text { Hartree) }\end{array}$ & $\begin{array}{l}\mathbf{H}_{\text {corr }} \\
(\text { Hartree) }\end{array}$ & $\begin{array}{l}\mathbf{S C F}\left(\mathrm{CH}_{2} \mathbf{C l}_{2}\right) \\
(\text { Hartree) }\end{array}$ \\
\hline- & 2135 & 2037 & 1.147 & -0.008450 & 0.046449 & -2053.69126967 \\
\hline $\mathrm{B}\left(\mathrm{OCH}_{3}\right)_{3}$ & - & & & & & \\
\hline $\mathrm{Bpin}\left(\mathrm{CH}_{3}\right)$ & 2155 & 2056 & 1.144 & 0.194832 & 0.278346 & -2504.89710656 \\
\hline $\mathrm{B}\left(\mathrm{CH}_{3}\right)_{3}$ & 2155 & 2056 & 1.144 & 0.098460 & 0.169988 & -2198.31272603 \\
\hline $\mathrm{B}\left(\mathrm{CH}_{3}\right)_{2}(\mathrm{OPh})$ & 2160 & 2060 & 1.143 & 0.150256 & 0.232962 & -2465.31364933 \\
\hline $\mathrm{BF} F_{2}(\mathrm{Ph})$ & 2161 & 2062 & 1.143 & 0.079680 & 0.157131 & -2510.04740705 \\
\hline $\mathrm{BH}_{3}$ & 2155 & 2056 & 1.144 & 0.020296 & 0.080268 & -2080.34172224 \\
\hline $\mathrm{Al}_{3}\left(\mathrm{CH}_{3}\right)_{3}$ & 2158 & 2059 & 1.143 & 0.085477 & 0.163913 & -2415.93005482 \\
\hline $\mathrm{BF}_{3}$ & 2162 & 2062 & 1.143 & -0.001317 & 0.065004 & -2378.31118835 \\
\hline
\end{tabular}

Table S38. Formation energies and enthalpies of Lewis acid adducts of $\left[\mathrm{Ni}(\mathrm{CO})_{3}-\left(\mathrm{KO}^{-} \mathrm{NO}_{2}\right)\right]^{-}$.

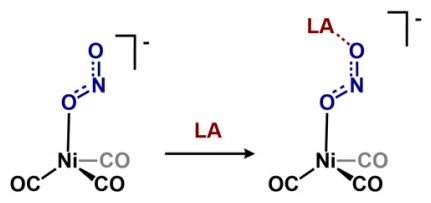

\begin{tabular}{|l|l|l|}
\hline Lewis acid & $\begin{array}{l}\Delta \mathbf{G} \\
\text { (kcal/mol) }\end{array}$ & $\begin{array}{l}\Delta \mathbf{H} \\
\text { (kcal/mol) }\end{array}$ \\
\hline $\mathrm{B}\left(\mathrm{OCH}_{3}\right)_{3}$ & & \\
\hline $\mathrm{Bpin}\left(\mathrm{CH}_{3}\right)$ & 20.1 & 7.5 \\
\hline $\mathrm{B}\left(\mathrm{CH}_{3}\right)_{3}$ & 8.7 & -5.2 \\
\hline $\mathrm{B}\left(\mathrm{CH}_{3}\right)_{2}(\mathrm{OPh})$ & 12.7 & -0.5 \\
\hline $\mathrm{BF}{ }_{2}(\mathrm{Ph})$ & -1.2 & -12.7 \\
\hline $\mathrm{BH}$ & -16.6 & -27.9 \\
\hline $\mathrm{Al}_{3}\left(\mathrm{CH}_{3}\right)_{3}$ & -9.3 & -22.3 \\
\hline $\mathrm{BF}_{3}$ & -13.5 & -25.5 \\
\hline
\end{tabular}


Table S39. Parameters of $\left[\mathrm{Ni}(\mathrm{CO})_{3}-\left(\mathrm{C}(\mathrm{O}) \mathrm{CH}_{3}\right)\right]^{-}$and adducts.

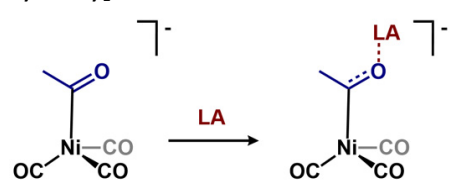

\begin{tabular}{|l|l|l|l|l|l|l|}
\hline Lewis acid & $\begin{array}{l}\mathbf{v} \mathbf{C O}_{\mathbf{a} 1} \\
\left(\mathbf{c m}^{-1}\right)\end{array}$ & $\begin{array}{l}\mathbf{v C O} \\
\text { calibrated }\end{array}$ & $\begin{array}{l}\text { Ave C-0 } \\
(\mathbf{A})\end{array}$ & $\begin{array}{l}\mathbf{G}_{\text {corr }} \\
(\text { Hartree) }\end{array}$ & $\begin{array}{l}\mathbf{H}_{\text {corr }} \\
(\text { Hartree) }\end{array}$ & $\begin{array}{l}\mathbf{S C F}\left(\mathbf{C H}_{\mathbf{2}} \mathbf{C l}_{\mathbf{2}}\right) \\
(\text { Hartree) }\end{array}$ \\
\hline- & 2086 & 1990 & 1.153 & 0.026644 & 0.082325 & -2001.77614518 \\
\hline $\mathrm{B}\left(\mathrm{OCH}_{3}\right)_{3}$ & 2119 & 2021 & 1.148 & 0.146813 & 0.226778 & -2372.16393826 \\
\hline $\mathrm{Bpin}\left(\mathrm{CH}_{3}\right)$ & 2126 & 2028 & 1.147 & 0.186763 & 0.268978 & -2413.39972229 \\
\hline $\mathrm{B}\left(\mathrm{CH}_{3}\right)_{3}$ & 2117 & 2020 & 1.149 & 0.134396 & 0.206021 & -2146.39777329 \\
\hline $\mathrm{B}\left(\mathrm{CH}_{3}\right)_{2}(\mathrm{OPh})$ & 2126 & 2028 & 1.147 & 0.186763 & 0.268978 & -2413.39972229 \\
\hline $\mathrm{BF}_{2}(\mathrm{Ph})$ & 2127 & 2029 & 1.147 & 0.116104 & 0.193324 & -2458.13785885 \\
\hline $\mathrm{BH}_{3}$ & 2118 & 2021 & 1.148 & 0.056729 & 0.116211 & -2028.42805729 \\
\hline $\mathrm{Al}_{3}\left(\mathrm{CH}_{3}\right)_{3}$ & 2121 & 2023 & 1.148 & 0.120424 & 0.199826 & -2364.01762008 \\
\hline $\mathrm{BF}_{3}$ & 2129 & 2031 & 1.147 & 0.035131 & 0.101117 & -2326.40271658 \\
\hline
\end{tabular}

Table S40. Formation energies and enthalpies of Lewis acid adducts of $\left[\mathrm{Ni}(\mathrm{CO})_{3}-\left(\mathrm{C}(\mathrm{O}) \mathrm{CH}_{3}\right)\right]^{-}$.

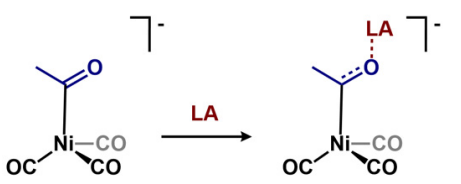

\begin{tabular}{|l|l|l|}
\hline Lewis acid & $\begin{array}{l}\Delta \mathbf{G} \\
\text { (kcal/mol) }\end{array}$ & $\begin{array}{l}\boldsymbol{\Delta} \\
\text { (kcal/mol) }\end{array}$ \\
\hline $\mathrm{B}\left(\mathrm{OCH}_{3}\right)_{3}$ & 19.1 & 4.9 \\
\hline $\mathrm{Bpin}\left(\mathrm{CH}_{3}\right)$ & 12.8 & -1.2 \\
\hline $\mathrm{B}\left(\mathrm{CH}_{3}\right)_{3}$ & 9.2 & -5.2 \\
\hline $\mathrm{B}\left(\mathrm{CH}_{3}\right)_{2}(\mathrm{OPh})$ & 12.8 & -1.2 \\
\hline $\mathrm{BF} 2(\mathrm{Ph})$ & -3.9 & -16.0 \\
\hline $\mathrm{BH}_{3}$ & -16.7 & -28.8 \\
\hline $\mathrm{Al}_{(}\left(\mathrm{CH}_{3}\right)_{3}$ & -11.1 & -24.0 \\
\hline $\mathrm{BF}_{3}$ & -16.8 & -29.6 \\
\hline
\end{tabular}


Binding affinity comparisons of anions and complexes

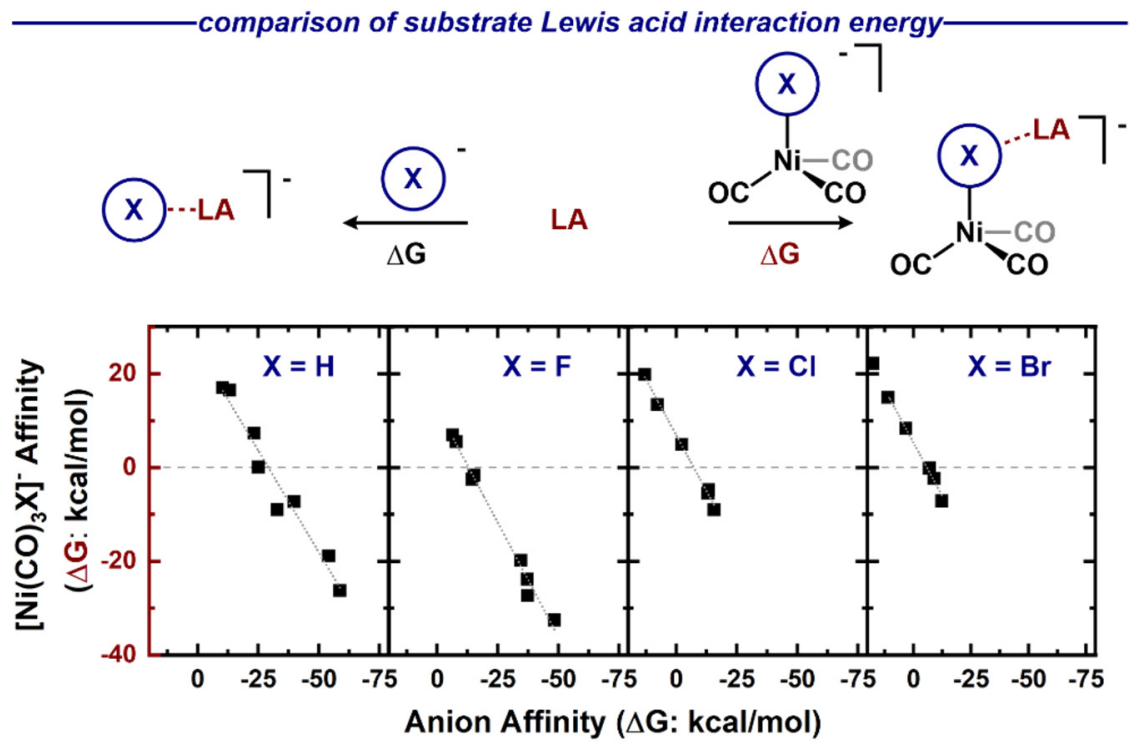

Figure S7. Lewis acid binding free energy to $\left[\mathrm{Ni}(\mathrm{CO})_{3}-\mathrm{X}\right]^{-}$as a function of $\mathrm{X}$ anion affinity.

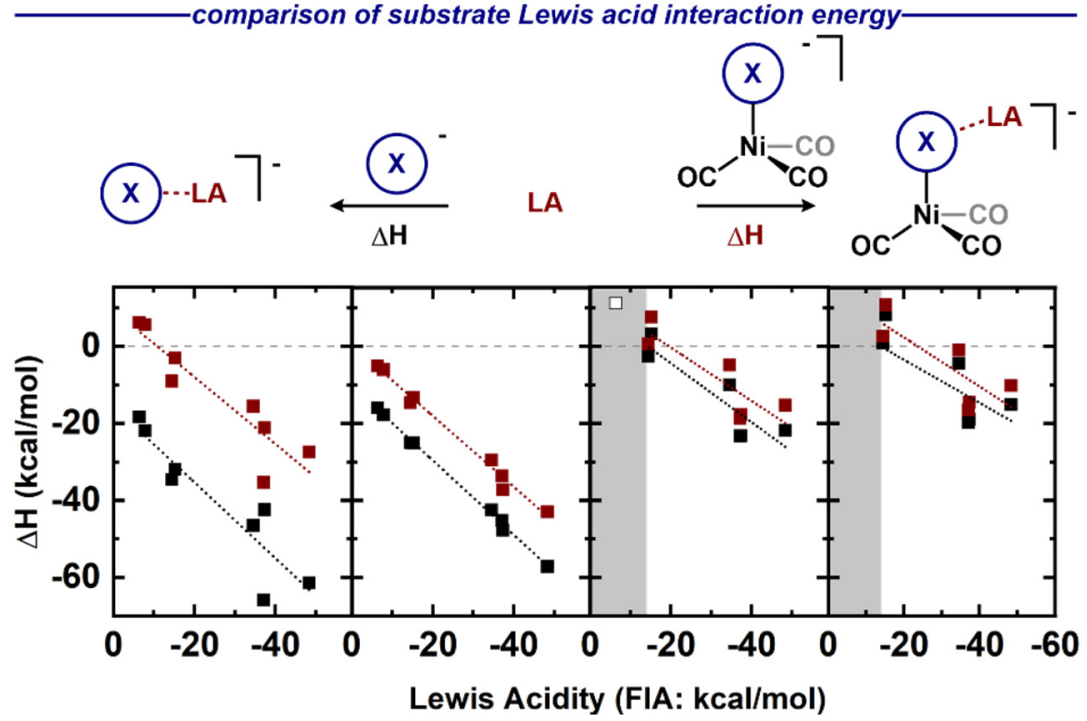

Figure S8. Lewis acid binding enthalpies to $\left[\mathrm{Ni}(\mathrm{CO})_{3}-\mathrm{X}\right]^{-}$as a function of Lewis acidity. The gray shaded areas reflect where Lewis acid interactions were insufficiently strong to model for complexes and any Lewis acid anion adducts. 


\section{Lewis acid interactions to $\mathrm{N}$-heterocycles}

Table S41. Bond distance comparisons for $\mathrm{BF}_{3}$ adducts of $\left[\mathrm{Ni}(\mathrm{CO})_{3}-(\mathrm{L})\right]^{\mathrm{n}}$.

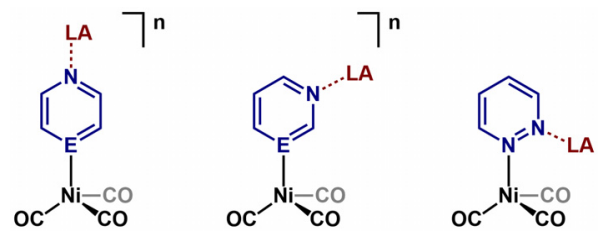

\begin{tabular}{|c|c|c|c|c|c|c|}
\hline L & $\begin{array}{l}\text { Parent } \\
\text { Ni-N (Å) }\end{array}$ & $\begin{array}{l}\text { Adduct } \\
\text { Ni-N (Å) }\end{array}$ & $\Delta \mathrm{Ni}-\mathrm{N}(\AA ̊)$ & N-B (Å) & $\Delta v_{\mathrm{CO}} \mathrm{cm}^{-1}$ & $\begin{array}{c}\text { Adduct formation } \\
\Delta \mathrm{H} \mathrm{kcal} / \mathrm{mol}\end{array}$ \\
\hline Pyrazine $\left(4-\mathrm{C}_{4} \mathrm{H}_{4} \mathrm{~N}_{2}\right)$ & 2.058 & 2.061 & 0.003 & 1.678 & -7 & -22.2 \\
\hline Pyrimidine $\left(3-\mathrm{C}_{4} \mathrm{H}_{4} \mathrm{~N}_{2}\right)$ & 2.083 & 2.094 & 0.011 & 1.680 & -9 & -21.9 \\
\hline Pyridazine $\left(2-\mathrm{C}_{4} \mathrm{H}_{4} \mathrm{~N}_{2}\right)$ & 2.055 & 2.116 & 0.061 & 1.697 & -12 & -17.9 \\
\hline$\left(4-\mathrm{C}_{5} \mathrm{H}_{4} \mathrm{~N}\right)^{-}$ & 2.003 & 1.988 & -0.015 & 1.600 & -15 & -38.5 \\
\hline$\left(3-\mathrm{C}_{5} \mathrm{H}_{4} \mathrm{~N}\right)^{-}$ & 2.009 & 2.004 & -0.005 & 1.609 & -17 & -37.8 \\
\hline
\end{tabular}


Lewis acid interactions to hydrazine

Table S42. Wiberg bond index and natural charge analysis of $\left[\mathrm{Ni}(\mathrm{CO})_{3}-\left(\mathrm{N}_{2} \mathrm{H}_{4}\right)\right]$.

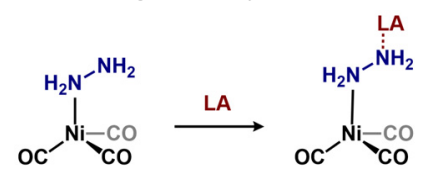

\begin{tabular}{|l|l|l|l|l|l|l|l|}
\hline & \multicolumn{3}{|l|}{ Wiberg bond indices } & \multicolumn{4}{l|}{ Natural Charge (e-) } \\
\hline Lewis acid & Ni- $\mathbf{N}_{\boldsymbol{\alpha}}$ & $\mathbf{N}-\mathbf{N}$ & & $\mathbf{N i}$ & $\mathbf{N}_{\boldsymbol{\alpha}}$ & $\mathbf{N}_{\boldsymbol{\beta}}$ & \\
\hline none & 0.40 & 1.04 & & -0.89 & -0.56 & -0.67 & \\
\hline Bpin $\left(\mathrm{CH}_{3}\right)$ & 0.40 & 1.01 & & -0.89 & -0.55 & -0.57 & \\
\hline $\mathrm{B}\left(\mathrm{OCH}_{3}\right)_{3}$ & 0.38 & 1.02 & & -0.88 & -0.56 & -0.57 & \\
\hline $\mathrm{B}\left(\mathrm{CH}_{3}\right)_{3}$ & 0.39 & 1.00 & & -0.89 & -0.54 & -0.53 & \\
\hline $\mathrm{B}\left(\mathrm{CH}_{3}\right)_{2}(\mathrm{OPh})$ & 0.39 & 1.01 & & -0.88 & -0.54 & -0.55 & \\
\hline $\mathrm{BF}_{2}(\mathrm{Ph})$ & 0.39 & 1.01 & & -0.88 & -0.54 & -0.56 & \\
\hline $\mathrm{BH}_{3}$ & 0.39 & 1.00 & & -0.89 & -0.54 & -0.50 & \\
\hline $\mathrm{Al}_{(}\left(\mathrm{CH}_{3}\right)_{3}$ & 0.40 & 1.01 & & -0.89 & -0.55 & -0.67 & \\
\hline $\mathrm{BF}_{3}$ & 0.36 & 1.02 & & -0.87 & -0.56 & -0.59 & \\
\hline
\end{tabular}

Table S43. Lewis acid acidification of $\mathrm{N}_{2} \mathrm{H}_{4}$.

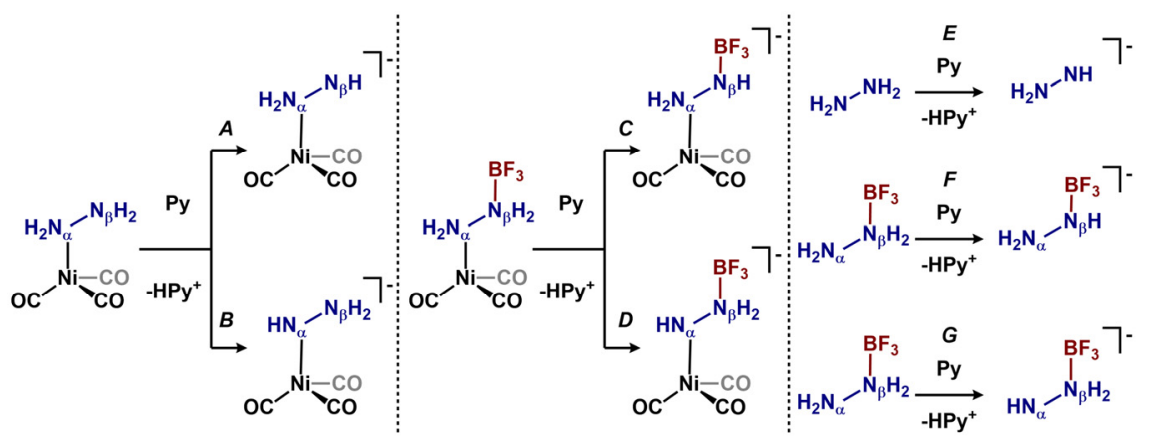

\begin{tabular}{|c|c|c|c|c|c|c|c|}
\hline Compound & $\begin{array}{l}\mathrm{SCF}\left(\mathrm{CH}_{3} \mathrm{CN}\right) \\
\text { (Hartree) }\end{array}$ & $\mathbf{G}_{\text {corr }}$ & Reaction & $\Delta \mathbf{G}$ & $K_{\mathrm{a}}$ & $\mathrm{p} K_{\mathrm{a}}$ & $\begin{array}{l}\mathrm{p} K_{\mathrm{a}} \\
\left(\mathrm{CH}_{3} \mathrm{CN}\right)\end{array}$ \\
\hline Pyridine (Py) & -248.289181 & 0.061913 & & & & & \\
\hline Pyridinium $\left(\mathrm{HPy}^{+}\right)$ & -248.736450 & 0.076199 & & & & & 12.33 \\
\hline $\mathrm{Ni}(\mathrm{CO})_{3}\left(\mathrm{NH}_{2} \mathrm{NH}_{2}\right)$ & -1960.327235 & 0.039495 & & & & & \\
\hline $\mathrm{Ni}(\mathrm{CO})_{3}\left(\mathrm{NH}_{2} \mathrm{NH}\right)^{-}$ & -1959.790237 & 0.027269 & $\mathrm{~A}$ & 57.6 & $1.18 \mathrm{E}+25$ & 42.2 & 54.5 \\
\hline $\mathrm{Ni}(\mathrm{CO})_{3}\left(\mathrm{NHNH}_{2}\right)^{-}$ & -1959.804468 & 0.026104 & $\mathrm{~B}$ & 47.9 & $1.41 \mathrm{E}+32$ & 35.1 & 47.5 \\
\hline $\mathrm{Ni}(\mathrm{CO})_{3}\left(\mathrm{NH}_{2} \mathrm{NH}_{2}-\mathrm{BF}_{3}\right)$ & -2284.946648 & 0.051080 & & & & & \\
\hline $\mathrm{Ni}(\mathrm{CO})_{3}\left(\mathrm{NH}_{2} \mathrm{NH}-\mathrm{BF}_{3}\right)^{-}$ & -2284.485946 & 0.034080 & $\mathrm{C}$ & 6.7 & $2.23 E+62$ & 4.9 & 17.3 \\
\hline $\mathrm{Ni}(\mathrm{CO})_{3}\left(\mathrm{NHNH}_{2}-\mathrm{BF}_{3}\right)^{-}$ & -2284.453220 & 0.036823 & $\mathrm{D}$ & 29.0 & $1.09 E+46$ & 21.2 & 33.6 \\
\hline $\mathrm{NH}_{2} \mathrm{NH}_{2}$ & -111.885478 & -111.854135 & & & & & \\
\hline $\mathrm{NH}_{2} \mathrm{NH}^{-}$ & -111.334159 & -111.318473 & $E$ & 64.4 & $1.16 \mathrm{E}+20$ & 47.2 & 59.5 \\
\hline $\mathrm{NH}_{2} \mathrm{NH}_{2}-\mathrm{BF}_{3}$ & -436.514226 & -436.474556 & & & & & \\
\hline $\mathrm{NHNH}_{2}-\mathrm{BF}_{3}^{-}$ & -435.996998 & -435.972291 & $\mathrm{~F}$ & 43.4 & $2.63 E+35$ & 31.9 & 44.2 \\
\hline $\mathrm{NH}_{2} \mathrm{NH}-\mathrm{BF}_{3}^{-}$ & -436.038613 & -436.012884 & $\mathrm{G}$ & 18.0 & $1.22 \mathrm{E}+54$ & 13.2 & 25.5 \\
\hline
\end{tabular}




\section{Lewis acid interactions to nitrite}

Table S44. Wiberg bond index and natural charge analysis of $\mathrm{k}-\mathrm{N} \mathrm{NO}_{2}$.

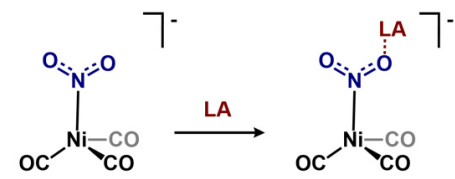

\begin{tabular}{|l|l|l|l|l|l|l|l|}
\hline & \multicolumn{5}{|l|}{ Wiberg bond indices } & \multicolumn{4}{l|}{ Natural Charge (e) } \\
\hline Lewis acid & $\mathbf{N i}-\mathbf{N}$ & $\mathbf{N}-\mathbf{O}_{\boldsymbol{\beta}}$ & $\mathbf{N}-\mathbf{O}_{\boldsymbol{\alpha}}$ & $\mathbf{N i}$ & $\mathbf{N}$ & $\mathbf{O}_{\boldsymbol{\beta}}$ & $\mathbf{O}_{\boldsymbol{\alpha}}$ \\
\hline none & 0.52 & 1.53 & 1.53 & -0.990 & 0.397 & -0.514 & -0.513 \\
\hline Bpin $\left(\mathrm{CH}_{3}\right)$ & 0.49 & 1.66 & 1.33 & -0.939 & 0.440 & -0.388 & -0.463 \\
\hline $\mathrm{B}\left(\mathrm{OCH}_{3}\right)_{3}$ & 0.52 & 1.53 & 1.52 & -0.983 & 0.406 & -0.504 & -0.503 \\
\hline $\mathrm{B}\left(\mathrm{CH}_{3}\right)_{3}$ & 0.49 & 1.66 & 1.32 & -0.937 & 0.441 & -0.385 & -0.441 \\
\hline $\mathrm{B}\left(\mathrm{CH}_{3}\right)_{2}(\mathrm{OPh})$ & 0.49 & 1.68 & 1.3 & -0.928 & 0.442 & -0.376 & -0.446 \\
\hline $\mathrm{BF}_{2}(\mathrm{Ph})$ & 0.49 & 1.73 & 1.24 & -0.927 & 0.450 & -0.342 & -0.488 \\
\hline $\mathrm{BH}_{3}$ & 0.49 & 1.68 & 1.29 & -0.934 & 0.436 & -0.372 & -0.418 \\
\hline $\mathrm{Al}_{(}\left(\mathrm{CH}_{3}\right)_{3}$ & 0.50 & 1.66 & 1.32 & -0.939 & 0.442 & -0.385 & -0.559 \\
\hline $\mathrm{BF}_{3}$ & 0.49 & 1.74 & 1.23 & -0.928 & 0.448 & -0.342 & -0.495 \\
\hline
\end{tabular}

Table S45. Wiberg bond index and natural charge analysis of $\mathrm{k}-\mathrm{O} \mathrm{NO}_{2}$.

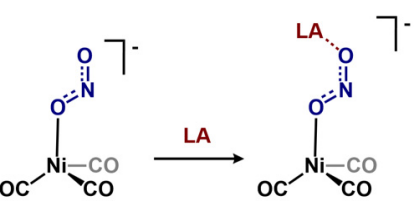

\begin{tabular}{|l|l|l|l|l|l|l|l|}
\hline & \multicolumn{3}{|l}{ Wiberg bond indices } & \multicolumn{3}{l|}{ Natural Charge (e-) } \\
\hline Lewis acid & $\mathbf{N i - \mathbf { O } _ { \boldsymbol { \alpha } }}$ & $\mathbf{O}_{\boldsymbol{\beta}}-\mathbf{N}$ & $\mathbf{N}-\mathbf{O}_{\boldsymbol{\alpha}}$ & $\mathbf{N i}$ & $\mathbf{N}$ & $\mathbf{O}_{\boldsymbol{\beta}}$ & $\mathbf{O}_{\boldsymbol{\alpha}}$ \\
\hline none & 0.38 & 1.41 & 1.64 & -0.821 & 0.270 & -0.502 & -0.501 \\
\hline Bpin $\left(\mathrm{CH}_{3}\right)$ & 0.33 & 1.42 & 1.56 & -0.780 & 0.343 & -0.385 & -0.450 \\
\hline $\mathrm{B}\left(\mathrm{OCH}_{3}\right)_{3}$ & 0.37 & 1.42 & 1.62 & -0.814 & 0.275 & -0.493 & -0.490 \\
\hline $\mathrm{B}\left(\mathrm{CH}_{3}\right)_{3}$ & 0.33 & 1.56 & 1.40 & -0.779 & 0.345 & -0.381 & -0.427 \\
\hline $\mathrm{B}\left(\mathrm{CH}_{3}\right)_{2}(\mathrm{OPh})$ & 0.32 & 1.58 & 1.39 & -0.772 & 0.359 & -0.373 & -0.437 \\
\hline $\mathrm{BF}_{2}(\mathrm{Ph})$ & 0.31 & 1.65 & 1.32 & -0.769 & 0.366 & -0.342 & -0.473 \\
\hline $\mathrm{BH}_{3}$ & 0.32 & 1.58 & 1.37 & -0.776 & 0.345 & -0.369 & -0.407 \\
\hline $\mathrm{Al}_{3}\left(\mathrm{CH}_{3}\right)_{3}$ & 0.32 & 1.57 & 1.41 & -0.777 & 0.347 & -0.380 & -0.548 \\
\hline $\mathrm{BF}_{3}$ & 0.31 & 1.66 & 1.32 & -0.768 & 0.366 & -0.343 & -0.485 \\
\hline
\end{tabular}



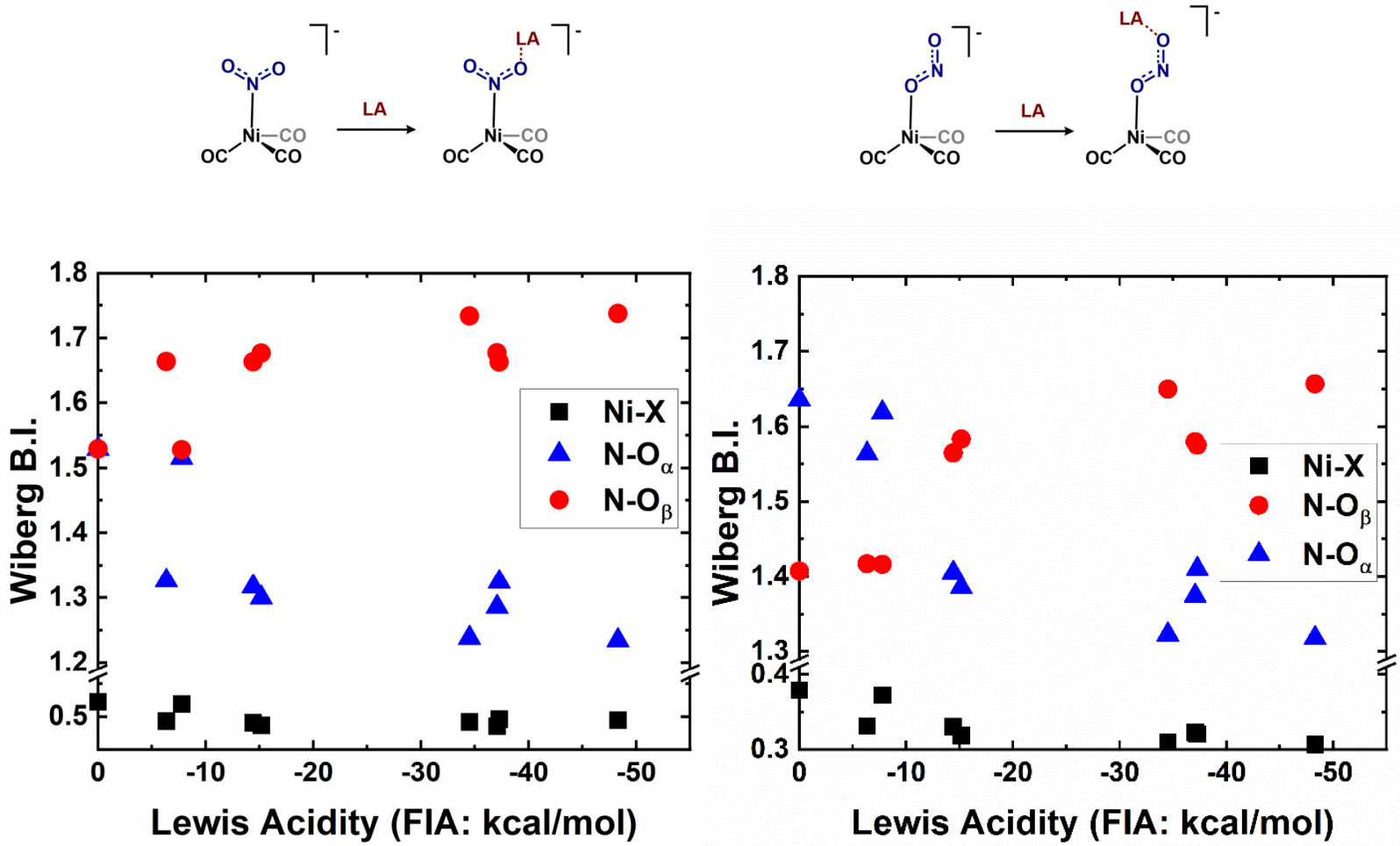

Figure S9. Wiberg bond indices of nitro (left) and nitrito (right) configuration of Lewis acid adducts of $\left[\mathrm{Ni}(\mathrm{CO})_{3}\left(\mathrm{NO}_{2}\right)\right]^{-}$complexes. 


\section{Lewis acid interactions to the acetyl ligand}

Table S46. Wiberg bond index and natural charge analysis of $-\mathrm{C}(\mathrm{O}) \mathrm{CH}_{3}$.

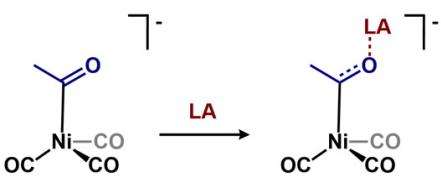

\begin{tabular}{|l|l|l|l|l|l|l|l|}
\hline & \multicolumn{3}{|l|}{ Wiberg bond indices } & \multicolumn{4}{l|}{ Natural Charge (e-) } \\
\hline Lewis acid & Ni-C & C-O & & Ni & C & O & \\
\hline none & 0.71 & 1.75 & & -1.279 & 0.487 & -0.674 & \\
\hline Bpin $\left(\mathrm{CH}_{3}\right)$ & 0.75 & 1.49 & & -1.215 & 0.536 & -0.636 & \\
\hline $\mathrm{B}\left(\mathrm{OCH}_{3}\right)_{3}$ & 0.76 & 1.45 & & -1.211 & 0.540 & -0.648 & \\
\hline $\mathrm{B}\left(\mathrm{CH}_{3}\right)_{3}$ & 0.75 & 1.50 & & -1.217 & 0.538 & -0.613 & \\
\hline $\mathrm{B}\left(\mathrm{CH}_{3}\right)_{2}(\mathrm{OPh})$ & 0.76 & 1.46 & & -1.206 & 0.535 & -0.620 & \\
\hline $\mathrm{BF}_{2}(\mathrm{Ph})$ & 0.77 & 1.41 & & -1.201 & 0.543 & -0.648 & \\
\hline $\mathrm{BH}_{3}$ & 0.75 & 1.46 & & -1.207 & 0.525 & -0.592 & \\
\hline $\mathrm{Al}_{3}\left(\mathrm{CH}_{3}\right)_{3}$ & 0.76 & 1.50 & & -1.225 & 0.538 & -0.742 & \\
\hline $\mathrm{BF}_{3}$ & 0.78 & 1.40 & & -1.200 & 0.536 & -0.654 & \\
\hline
\end{tabular}
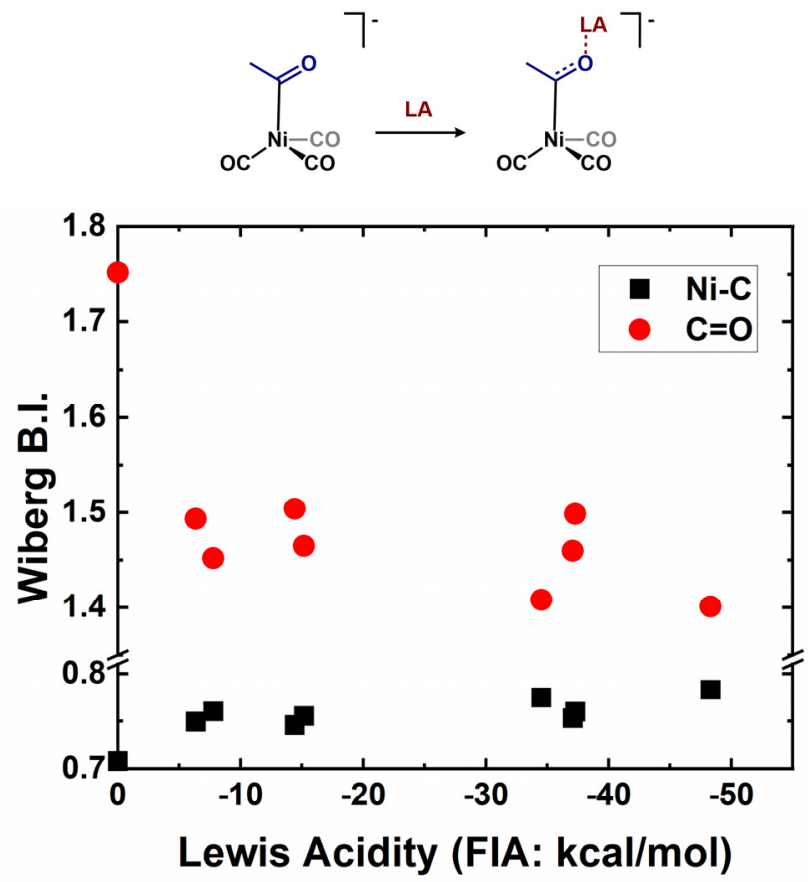

Figure S10. Wiberg bond indices of the acetyl ligand of $\left[\mathrm{Ni}(\mathrm{CO})_{3}\left(\mathrm{C}(\mathrm{O}) \mathrm{CH}_{3}\right)\right]^{-}$and corresponding Lewis acid adducts. 

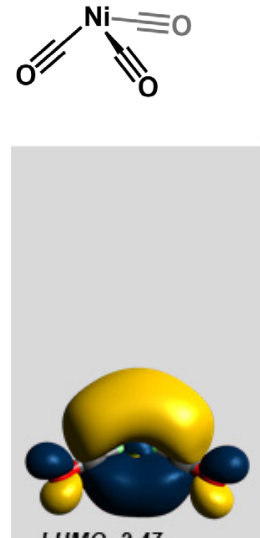

LUMO -2.47

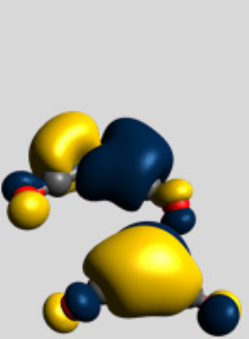

НОМО -7.10, НОМО-1

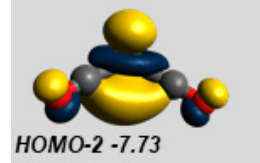

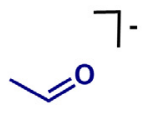
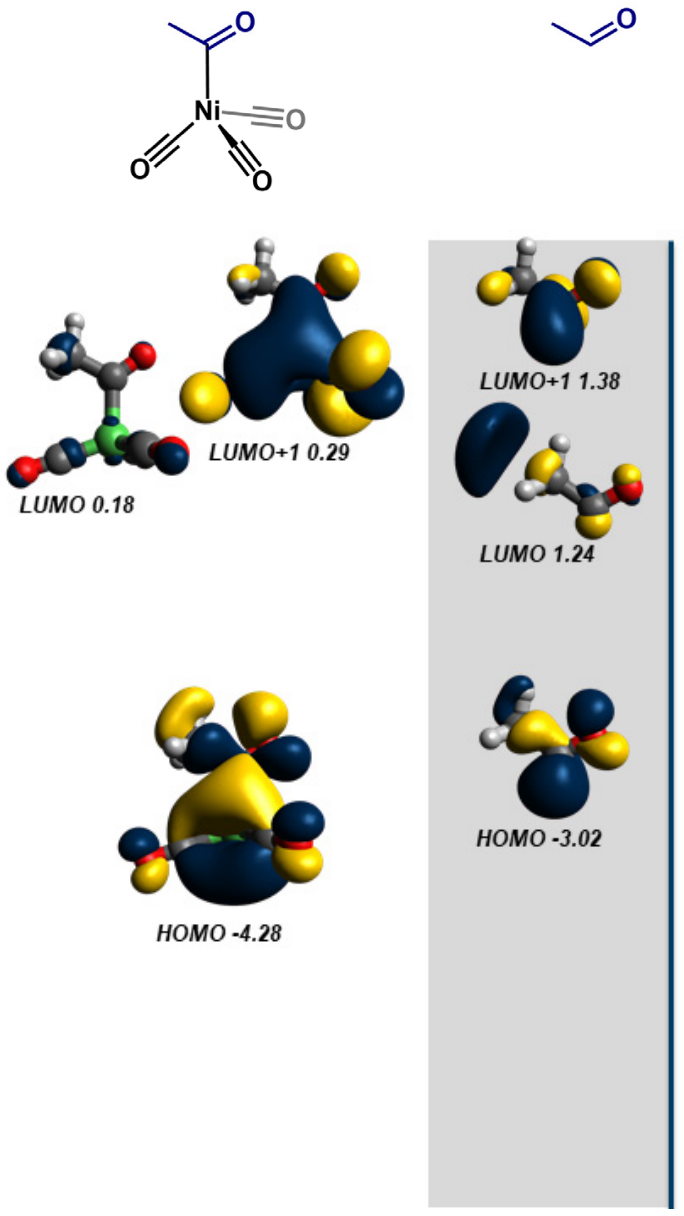
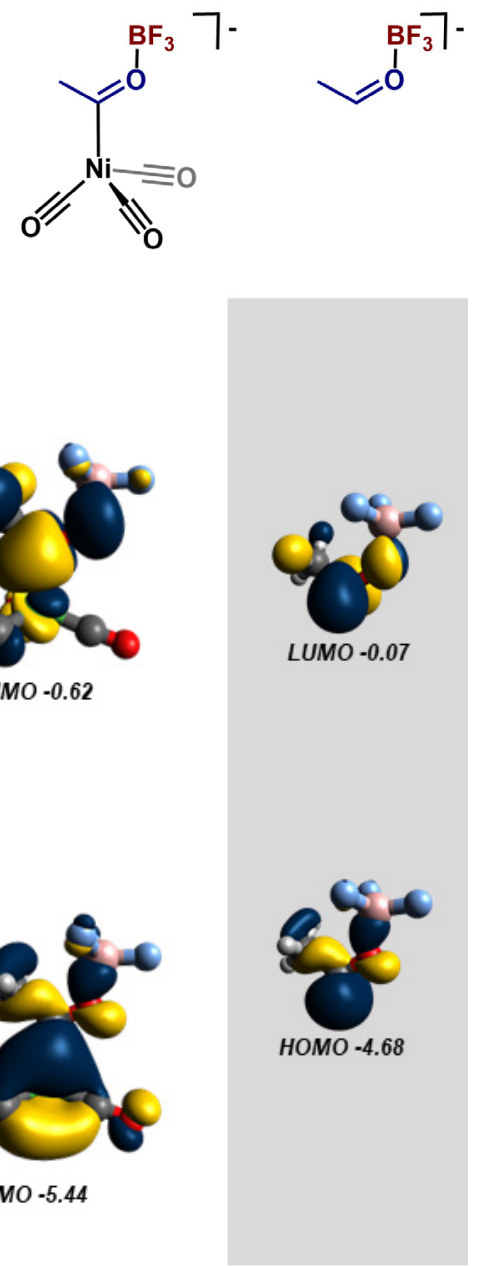

Figure S11. Molecular orbital renderings and energies (eV) for $\left[\mathrm{Ni}(\mathrm{CO})_{3}\left(\mathrm{C}(\mathrm{O}) \mathrm{CH}_{3}\right)\right]^{-}$(left) and $\mathrm{BF}_{3}$ adduct (right) with corresponding fragment orbitals in gray. 


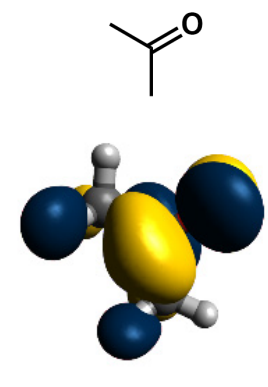

LUMO -0.51
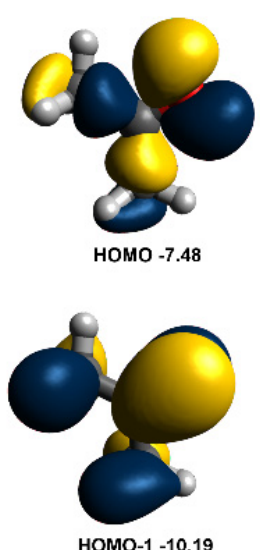
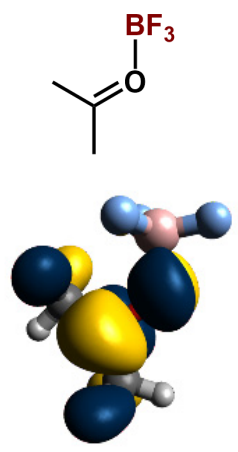

LUMO -1.99

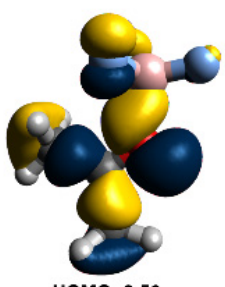

HOMO -9.56

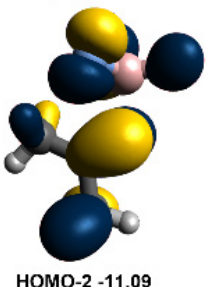

Figure S12. Molecular orbital renderings and energies (eV) for acetone (left) and $\mathrm{BF}_{3}$ adduct (right). 


\section{Lewis acid interactions to the cyanide ligand}

\section{Experimental Procedures General Considerations.}

Dichloromethane was sparged, dried through a Glass Contour solvent purification system through percolation through a $\mathrm{Cu}$ catalyst, molecular sieves, and alumina. The solvent was then stored in a glovebox of molecular sieves. $\mathrm{B}(\mathrm{OiPr})_{3}, \mathrm{BBu}, \mathrm{B}(\mathrm{OPh})_{3}, \mathrm{~B}(\mathrm{Ph})_{3}, \mathrm{Al}\left(\mathrm{CH}_{3}\right)_{3}$, and $\mathrm{OPEt}_{3}$ were commercially available were purchased anhydrous, stored in a dry glovebox atmosphere, and used without further purification. $\mathrm{B}\left(\mathrm{C}_{6} \mathrm{~F}_{5}\right)_{3}$ was sublimed prior to use. $\mathrm{BBN}(\mathrm{OPh})^{11}$ and $\mathrm{B}\left(\mathrm{C}_{6} \mathrm{H}_{3} \mathrm{~F}_{2}\right)_{3}{ }^{12}$ were prepared as previously reported.

[PPh $4\left[\mathrm{Ni}(\mathrm{CN})(\mathrm{CO})_{3}\right]$ (1 mmol scale in $\mathrm{NiCl}_{2}$ ) was prepared as previously reported ${ }^{13}$ in a fume hood (face velocity $81 \mathrm{ft} / \mathrm{min}$ at $6 \mathrm{ft}^{2}$ opening: $486 \mathrm{ft}^{3} / \mathrm{min}=13800 \mathrm{~L} / \mathrm{min}$ ) equipped with an external cO detector (GFG 4400 EC35 CO detector 0-500 ppm).

*Caution* Measures were taken to prevent liberation of $\mathrm{Ni}(\mathrm{CO})_{4}$, a potential highly toxic and volatile side product. Following extraction, the reaction contents were quenched by addition of $\mathrm{Br}_{2}$, followed by bleach. During manipulations requiring a positive atmosphere of $\mathrm{CO}$, the atmosphere was passed through a bleach bubbler (see below). AEGL (2009) risk to life $10 \mathrm{~min}$ at $0.46 \mathrm{ppm} ; 1 \mathrm{~h}$ at $0.16 \mathrm{ppm} .{ }^{14} \mathrm{NIOSH}$ : exposure limit $8 \mathrm{~h}$ TWA $=0.001 \mathrm{ppm} .^{15}$

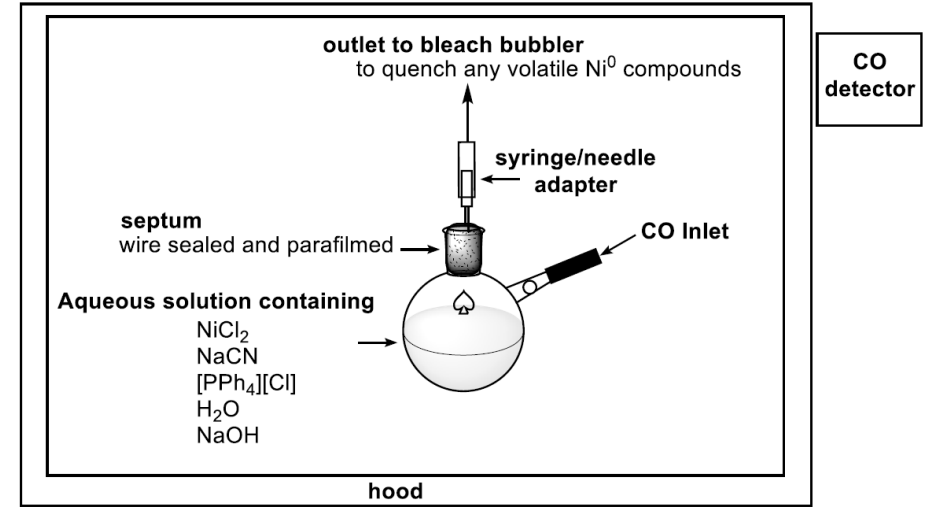

Figure S13. Schematic of $[\mathrm{PPN}]\left[\mathrm{Ni}(\mathrm{CO})_{3}(\mathrm{CN})\right]$ air-free handling.

\section{Procedure for formation of Lewis acid adducts of $\left[\mathrm{PPh}_{4}\right]\left[\mathrm{Ni}(\mathrm{CN})(\mathrm{CO})_{3}\right]$.}

Lewis acid stock solutions $(20 \mu \mathrm{L}, 0.26 \mathrm{M}, 0.05 \mathrm{mmol})$ in $\mathrm{CH}_{2} \mathrm{Cl}_{2}$ were combined with $0.3 \mathrm{~mL}$ aliquots of an $0.0175 \mathrm{M}\left[\mathrm{PPh}_{4}\right]\left[\mathrm{Ni}(\mathrm{CN})(\mathrm{CO})_{3}\right]$ stock solution $(0.05 \mathrm{mmol})$. The solutions were mixed, rapidly transferred to a $\mathrm{KBr}$ solution IR cell, removed from a glovebox, and IR spectra acquired. In a fume hood, the contents of the reaction mixture were flushed with additional dry solvent into a stirring bleach solution.

For the reaction with $\mathrm{Al}(\mathrm{CH} 3)_{3}$ the solution immediately became dark black with formation of a precipitate. Quickly filtering the solution in the glovebox prior to solution IR measurement only resulted in a peak at $2040 \mathrm{~cm}^{-1}\left(\mathrm{Ni}(\mathrm{CO})_{4}\right)$. 


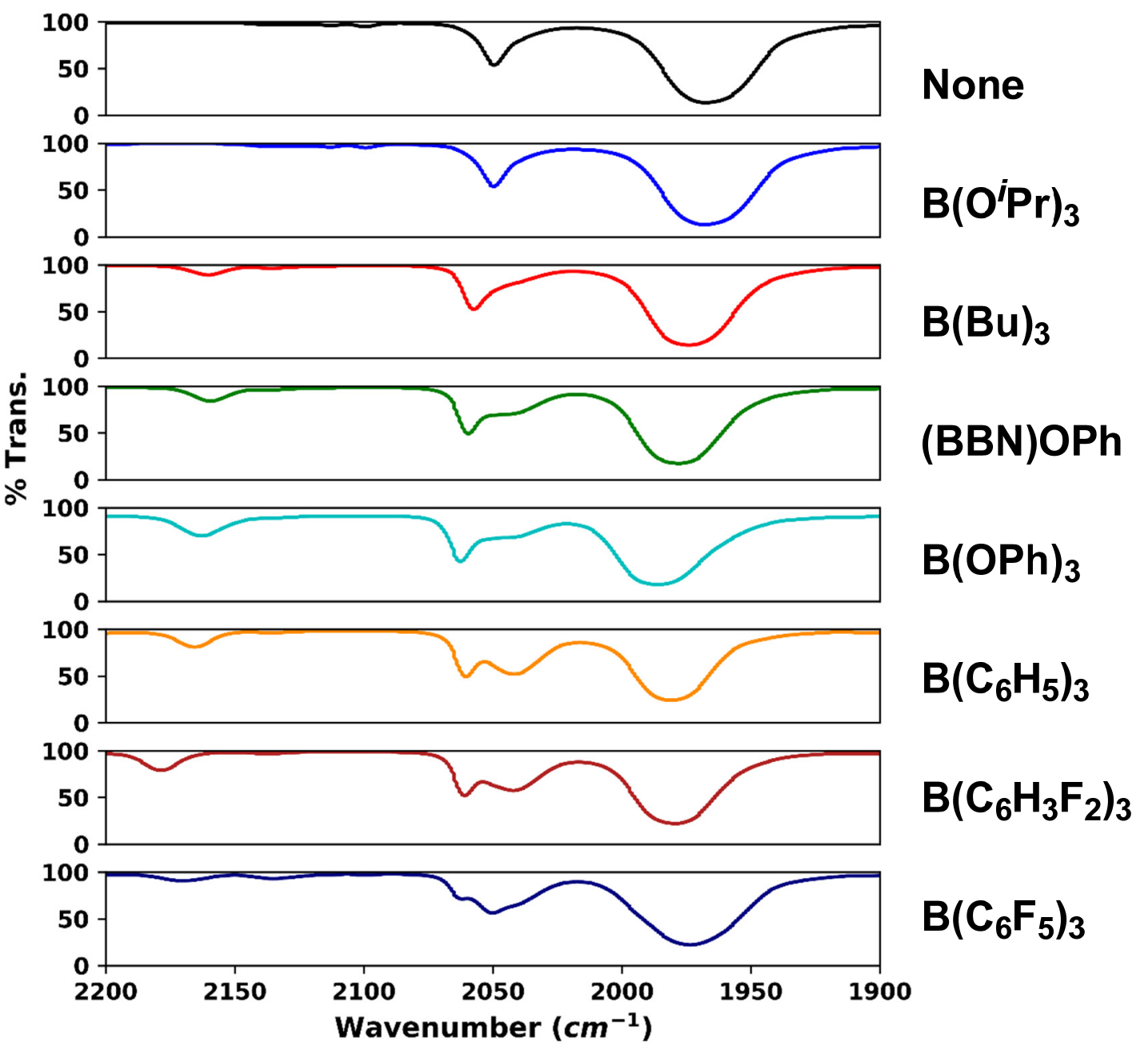

Figure S14. IR spectra $\left(\mathrm{CH}_{2} \mathrm{Cl}_{2}\right)$ of $\left[\mathrm{PPh}_{4}\right]\left[\mathrm{Ni}(\mathrm{CN})(\mathrm{CO})_{3}\right]$ with specified Lewis acid.

Table S47. Reported shifts of Lewis acid adducts of $\left[\mathrm{PPh}_{4}\right]\left[\mathrm{Ni}(\mathrm{CN})(\mathrm{CO})_{3}\right]$.

\begin{tabular}{|c|c|c|c|c|c|c|}
\hline Lewis acid & $\mathbf{v}_{\text {CO(e) }}\left(\mathbf{c m}^{-\mathbf{1}}\right)$ & $\boldsymbol{\Delta} \mathbf{v}_{\text {CO(e) }}\left(\mathbf{c m}^{-\mathbf{1}}\right)$ & $\mathbf{v}_{\text {CO(a) })}\left(\mathbf{c m}^{-\mathbf{1}}\right)$ & $\Delta \mathbf{v}_{\text {CO(a) })}\left(\mathbf{c m}^{-\mathbf{1}}\right)$ & $\mathbf{v}_{\mathbf{C N}}\left(\mathbf{c m}^{-\mathbf{1}}\right)$ & $\boldsymbol{\Delta v}_{\mathbf{C N}}\left(\mathbf{c m}^{-\mathbf{1}}\right)$ \\
\hline none & 1966 & 0 & 2048 & 0 & 2100 & 0 \\
\hline $\mathrm{B}\left(\mathrm{O}^{i} \mathrm{Pr}\right)_{3}$ & - & - & - & - & - & - \\
\hline $\mathrm{BBu}$ & 1974 & -8 & 2058 & -10 & 2160 & -60 \\
\hline $\mathrm{BBN}(\mathrm{OPh})$ & 1981 & -15 & 2060 & -12 & 2159 & -59 \\
\hline $\mathrm{B}(\mathrm{OPh})_{3}$ & 1986 & -20 & 2062 & -14 & 2163 & -63 \\
\hline $\mathrm{B}(\mathrm{Ph})_{3}$ & 1981 & -15 & 2060 & -12 & 2166 & -66 \\
\hline $\mathrm{B}\left(\mathrm{C}_{6} \mathrm{H}_{3} \mathrm{~F}_{2}\right)_{3}$ & 1979 & -13 & 2061 & -13 & 2179 & -79 \\
\hline $\mathrm{B}\left(\mathrm{C}_{6} \mathrm{~F}_{5}\right)_{3}$ & 1980 & -14 & 2062 & -14 & 2170 & -70 \\
\hline
\end{tabular}




\section{Procedure for measurement of acceptor number.}

Aliquots $(0.5 \mathrm{~mL})$ of an $0.055 \mathrm{M} \mathrm{O}=\mathrm{PEt}_{3} \mathrm{CH}_{2} \mathrm{Cl}_{2}$ stock solution were added to Lewis acids $(0.028 \mathrm{mmol})$ and transferred to an NMR tube. The solutions were mixed and transferred to NMR tubes. The instrument was referenced to external $85 \% \mathrm{H}_{3} \mathrm{PO}_{4}$ and ${ }^{31} \mathrm{P} \mathrm{NMR}$ spectra recorded. Acceptor number values were determined using the following formula:

$$
A N=2.21 \times(\delta-41.0)
$$

Table S48. Acceptor number data for measurement of Lewis acidity.

\begin{tabular}{|l|l|l|}
\hline Lewis acid & $\boldsymbol{\delta}^{31} \mathbf{P}$ & AN \\
\hline- & 51.0 & 22.1 \\
\hline $\mathrm{B}\left(\mathrm{O}^{i} \mathrm{Pr}\right)_{3}$ & 51.0 & 22.1 \\
\hline $\mathrm{BBu}$ & 51.0 & 22.1 \\
\hline $\mathrm{BBN}(\mathrm{OPh})$ & 54 & 28.7 \\
\hline $\mathrm{B}(\mathrm{OPh})_{3}$ & 70.1 & 64.3 \\
\hline $\mathrm{B}(\mathrm{Ph})_{3}$ & 72.2 & 69.0 \\
\hline $\mathrm{B}\left(\mathrm{C}_{6} \mathrm{H}_{3} \mathrm{~F}_{2}\right)_{3}$ & 72.8 & 70.3 \\
\hline $\mathrm{B}\left(\mathrm{C}_{6} \mathrm{~F}_{5}\right)_{3}$ & 77.6 & 80.9 \\
\hline $\mathrm{Al}\left(\mathrm{CH}_{3}\right)_{3}$ & 68.7 & 61.1 \\
\hline
\end{tabular}




\section{Computational analysis of Lewis acid effects to cyanide}

Table S49. Parameters of $\left[\mathrm{Ni}(\mathrm{CO})_{3}(\mathrm{CN})\right]^{-}$and adducts.

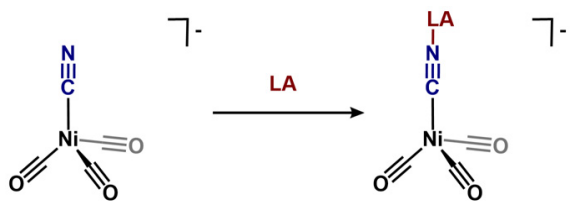

\begin{tabular}{|c|c|c|c|c|c|c|}
\hline Lewis acid & $\begin{array}{l}\mathrm{vCO}_{\mathrm{a} 1} \\
\left(\mathrm{~cm}^{-1}\right)\end{array}$ & $\begin{array}{l}\mathrm{vCO}_{\mathrm{a} 1} \\
\left(\mathrm{~cm}^{-1}\right) \\
\text { calibrated }\end{array}$ & $\begin{array}{l}\text { Ave C-O } \\
\text { (Å) }\end{array}$ & $\mathbf{G}_{\text {corr }}($ Hartree $)$ & $\mathrm{H}_{\text {corr }}($ Hartree) & $\operatorname{SCF}\left(\mathrm{CH}_{2} \mathrm{Cl}_{2}\right)$ \\
\hline- & 2122 & 2025 & 1.148 & -0.006772 & 0.042821 & -1941.41385483 \\
\hline $\mathrm{B}\left(\mathrm{OCH}_{3}\right)_{3}$ & 2144 & 2045 & 1.144 & 0.110064 & 0.186954 & -2311.80553615 \\
\hline $\mathrm{Bpin}\left(\mathrm{CH}_{3}\right)$ & 2143 & 2045 & 1.144 & 0.195056 & 0.274626 & -2392.62652147 \\
\hline $\mathrm{B}\left(\mathrm{CH}_{3}\right)_{3}$ & 2141 & 2043 & 1.144 & 0.098895 & 0.166533 & -2086.04852496 \\
\hline $\mathrm{B}\left(\mathrm{CH}_{3}\right)_{2}(\mathrm{OPh})$ & 2146 & 2047 & 1.144 & 0.150835 & 0.229387 & -2353.04755129 \\
\hline $\mathrm{BF}_{2}(\mathrm{Ph})$ & 2147 & 2048 & 1.143 & 0.079043 & 0.153624 & -2397.78068981 \\
\hline $\mathrm{BH}_{3}$ & 2140 & 2041 & 1.145 & 0.019469 & 0.076613 & -1968.07840490 \\
\hline $\mathrm{Al}\left(\mathrm{CH}_{3}\right)_{3}$ & 2143 & 2044 & 1.144 & 0.086640 & 0.160244 & -2303.66062727 \\
\hline $\mathrm{BF}_{3}$ & 2133 & 2035 & 1.143 & 0.001702 & 0.059962 & -2266.04372059 \\
\hline $\mathrm{B}\left(\mathrm{O}^{\prime} \mathrm{Pr}\right)_{3}$ & 2145 & 2046 & 1.144 & 0.268079 & 0.363467 & -2547.71525313 \\
\hline $\mathrm{BBu}_{3}$ & 2142 & 2044 & 1.144 & 0.33776 & 0.436022 & -2439.86358993 \\
\hline $\mathrm{BBN}(\mathrm{OPh})$ & 2147 & 2048 & 1.143 & 0.279719 & 0.366091 & -2586.50895361 \\
\hline $\mathrm{B}(\mathrm{OPh})_{3}$ & 2151 & 2052 & 1.142 & 0.254586 & 0.354735 & -2887.05485659 \\
\hline $\mathrm{B}(\mathrm{Ph})_{3}$ & 2147 & 2048 & 1.143 & 0.244481 & 0.338566 & -2661.26403423 \\
\hline $\mathrm{B}\left(\mathrm{C}_{6} \mathrm{H}_{3} \mathrm{~F}_{2}\right)_{3}$ & 2147 & 2048 & 1.143 & 0.190452 & 0.294568 & -3256.74401562 \\
\hline $\mathrm{B}\left(\mathrm{C}_{6} \mathrm{~F}_{5}\right)_{3}$ & 2153 & 2054 & 1.142 & 0.108455 & 0.230639 & -4149.86951279 \\
\hline
\end{tabular}

Table S50. Parameters of $\left[\mathrm{Ni}(\mathrm{CO})_{3}(\mathrm{CN})\right]^{-}$and adducts.

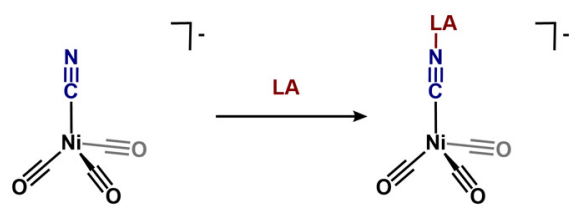

\begin{tabular}{|l|l|l|}
\hline Lewis acid & $\begin{array}{l}\Delta \mathbf{G} \\
\text { (kcal/mol) }\end{array}$ & $\begin{array}{l}\Delta \mathbf{H} \\
\text { (kcal/mol) }\end{array}$ \\
\hline $\mathrm{B}\left(\mathrm{OCH}_{3}\right)_{3}$ & 14.6 & 2.3 \\
\hline $\mathrm{Bpin}\left(\mathrm{CH}_{3}\right)$ & 15.0 & 3.2 \\
\hline $\mathrm{B}\left(\mathrm{CH}_{3}\right)_{3}$ & -0.3 & -13.4 \\
\hline $\mathrm{B}\left(\mathrm{CH}_{3}\right)_{2}(\mathrm{OPh})$ & 4.9 & -7.6 \\
\hline $\mathrm{BF}{ }_{2}(\mathrm{Ph})$ & -9.4 & -19.3 \\
\hline $\mathrm{BH}_{3}$ & -27.0 & -36.7 \\
\hline $\mathrm{Al}_{3}\left(\mathrm{CH}_{3}\right)_{3}$ & -14.6 & -27.4 \\
\hline $\mathrm{BF}{ }_{3}$ & -18.9 & -32.7 \\
\hline $\mathrm{B}\left(\mathrm{O}^{\prime} \mathrm{Pr}\right)_{3}$ & 19.2 & 7.2 \\
\hline $\mathrm{BBu}$ & 3.7 & -10.7 \\
\hline $\mathrm{BBN}(\mathrm{OPh})$ & 3.4 & -8.8 \\
\hline $\mathrm{B}\left(\mathrm{OPh}_{3}\right)_{3}$ & 1.0 & -11.6 \\
\hline $\mathrm{B}(\mathrm{Ph})_{3}$ & -6.7 & -16.4 \\
\hline $\mathrm{B}\left(\mathrm{C}_{6} \mathrm{H}_{3} \mathrm{~F}_{2}\right)_{3}$ & -9.6 & -21.2 \\
\hline $\mathrm{B}\left(\mathrm{C}_{6} \mathrm{~F}_{5}\right)_{3}$ & -20.7 & -32.5 \\
\hline
\end{tabular}


Table S51. Parameters of $\left[\mathrm{Ni}(\mathrm{CO})_{3}(\mathrm{NC})\right]^{-}$isomer and selected adducts.

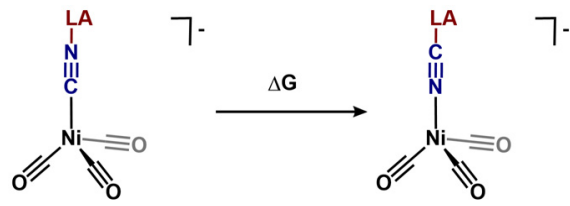

\begin{tabular}{|l|l|l|l|}
\hline Lewis acid & $\mathbf{G}_{\text {corr }}($ Hartree $)$ & $\operatorname{SCF}\left(\mathrm{CH}_{\mathbf{2}} \mathrm{Cl}_{2}\right)$ & $\begin{array}{l}\text { Isomer } \\
\mathbf{\Delta} \\
\text { (kcal/mol) }\end{array}$ \\
\hline- & -0.00831 & -1941.39330345 & 11.9 \\
\hline $\mathrm{B}\left(\mathrm{CH}_{3}\right)_{3}$ & 0.13367 & -2086.04243421 & 25.6 \\
\hline $\mathrm{BF}_{3}$ & -0.00307 & -2266.03003052 & 5.6 \\
\hline
\end{tabular}

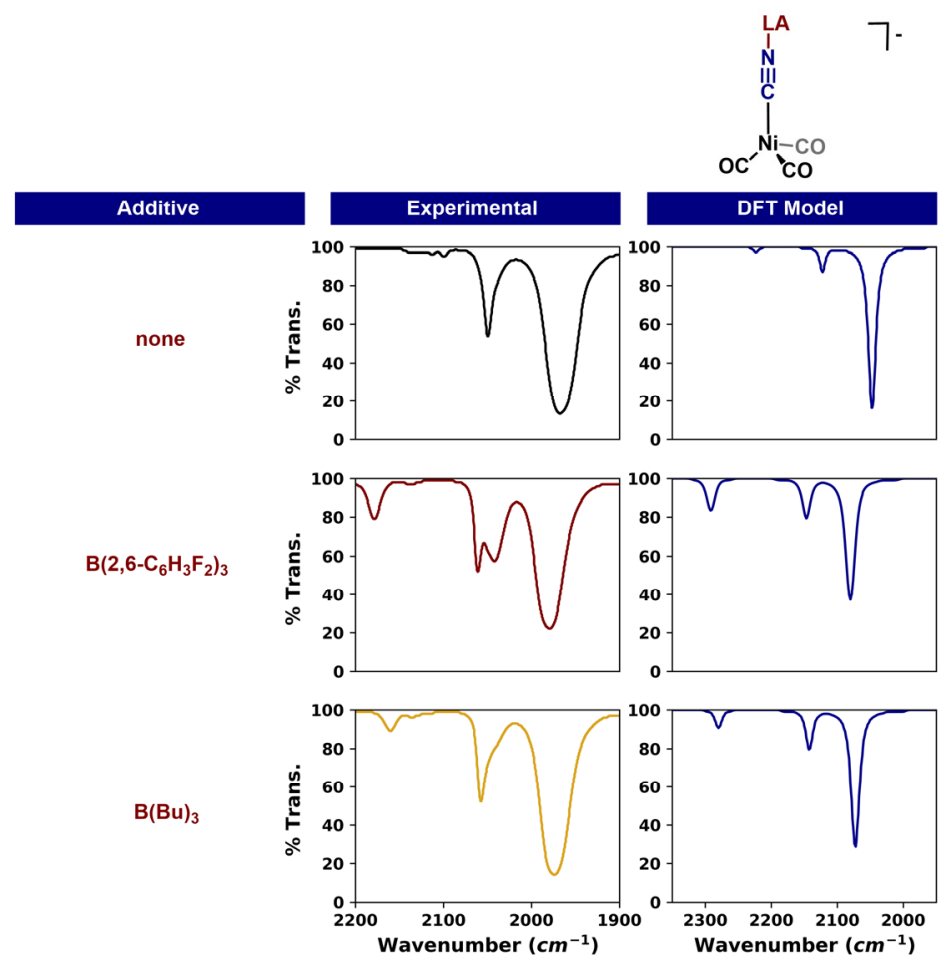

Figure S15. Representative experimental solution spectra and calculated IR spectra. 
Table S52. Bond distances and natural charge analysis of $\mathrm{CN}^{-}$of $\left[\mathrm{Ni}(\mathrm{CO})_{3}(\mathrm{CN})\right]^{-}$and adducts.

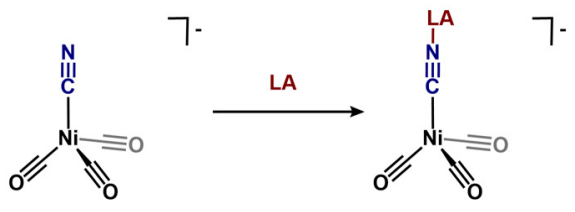

\begin{tabular}{|c|c|c|c|c|c|}
\hline \multirow[b]{2}{*}{ Lewis acid } & \multicolumn{2}{|c|}{ Bond Distances (Å) } & \multicolumn{3}{|c|}{ Natural Charge (e') } \\
\hline & $\mathrm{Ni}-\mathrm{C}$ & $\mathrm{C}-\mathrm{N}$ & $\mathrm{Ni}$ & C & $\mathbf{N}$ \\
\hline none & 1.9468 & 1.1648 & -1.24 & 0.18 & -0.64 \\
\hline $\operatorname{Bpin}\left(\mathrm{CH}_{3}\right)$ & 1.9264 & 1.1590 & -1.22 & 0.40 & -0.56 \\
\hline $\mathrm{B}\left(\mathrm{OCH}_{3}\right)_{3}$ & 1.9257 & 1.1585 & -1.22 & 0.41 & -0.57 \\
\hline $\mathrm{B}\left(\mathrm{CH}_{3}\right)_{3}$ & 1.9309 & 1.1590 & -1.22 & 0.38 & -0.51 \\
\hline $\mathrm{B}\left(\mathrm{CH}_{3}\right)_{2}(\mathrm{OPh})$ & 1.9242 & 1.1585 & -1.22 & 0.42 & -0.54 \\
\hline $\mathrm{BF}_{2}(\mathrm{Ph})$ & 1.9194 & 1.1591 & -1.22 & 0.44 & -0.59 \\
\hline $\mathrm{BH}_{3}$ & 1.9324 & 1.1582 & -1.22 & 0.38 & -0.50 \\
\hline $\mathrm{Al}\left(\mathrm{CH}_{3}\right)_{3}$ & 1.9232 & 1.1627 & -1.22 & 0.39 & -0.71 \\
\hline $\mathrm{BF}_{3}$ & 1.9196 & 1.1581 & -1.22 & 0.45 & -0.62 \\
\hline $\mathrm{B}\left(\mathrm{O}^{i} \mathrm{Pr}\right)_{3}$ & 1.9242 & 1.5886 & -1.22 & 0.41 & -0.58 \\
\hline $\mathrm{BBu}_{3}$ & 1.9292 & 1.1594 & -1.22 & 0.38 & -0.51 \\
\hline $\mathrm{BBN}(\mathrm{OPh})$ & 1.9203 & 1.1605 & -1.21 & 0.42 & -0.56 \\
\hline $\mathrm{B}(\mathrm{OPh})_{3}$ & 1.9100 & 1.6035 & -1.21 & 0.47 & -0.60 \\
\hline $\mathrm{B}(\mathrm{Ph})_{3}$ & 1.9205 & 1.1600 & -1.21 & 0.43 & -0.52 \\
\hline $\mathrm{B}\left(\mathrm{C}_{6} \mathrm{H}_{3} \mathrm{~F}_{2}\right)_{3}$ & 1.9154 & 1.1588 & -1.21 & 0.46 & -0.54 \\
\hline $\mathrm{B}\left(\mathrm{C}_{6} \mathrm{~F}_{5}\right)_{3}$ & 1.9049 & 1.1610 & -1.21 & 0.48 & -0.57 \\
\hline
\end{tabular}

Table S53. Relevant bond parameters of $\left[\mathrm{Ni}(\mathrm{CO})_{3}\left(\mathrm{CN}^{\left.-B F_{3}\right)}\right]^{-}\right.$upon $\mathrm{C}-\mathrm{N}-\mathrm{B}$ bend.
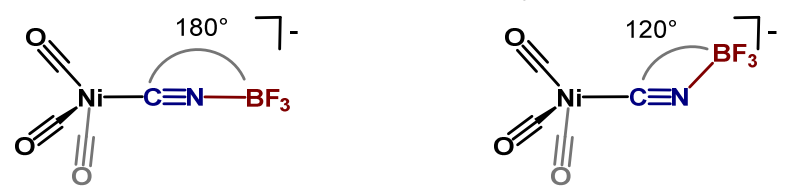

\begin{tabular}{|l|l|l|l|l|l|l|}
\hline & \multicolumn{3}{|l|}{ Bond distances (合) } & \multicolumn{3}{l|}{ Wiberg bond indices } \\
\hline Angle & Ni-C & C-N & N-B & Ni-C & C-N & N-B \\
\hline 120 & 1.895 & 1.172 & 1.644 & 0.85 & 2.50 & 0.57 \\
\hline 130 & 1.900 & 1.168 & 1.621 & 0.85 & 2.51 & 0.59 \\
\hline 140 & 1.905 & 1.165 & 1.604 & 0.84 & 2.53 & 0.61 \\
\hline 150 & 1.909 & 1.162 & 1.592 & 0.83 & 2.55 & 0.62 \\
\hline 160 & 1.913 & 1.160 & 1.583 & 0.83 & 2.56 & 0.62 \\
\hline 170 & 1.917 & 1.159 & 1.577 & 0.82 & 2.56 & 0.62 \\
\hline 180 & 1.919 & 1.158 & 1.575 & 0.82 & 2.57 & 0.63 \\
\hline
\end{tabular}



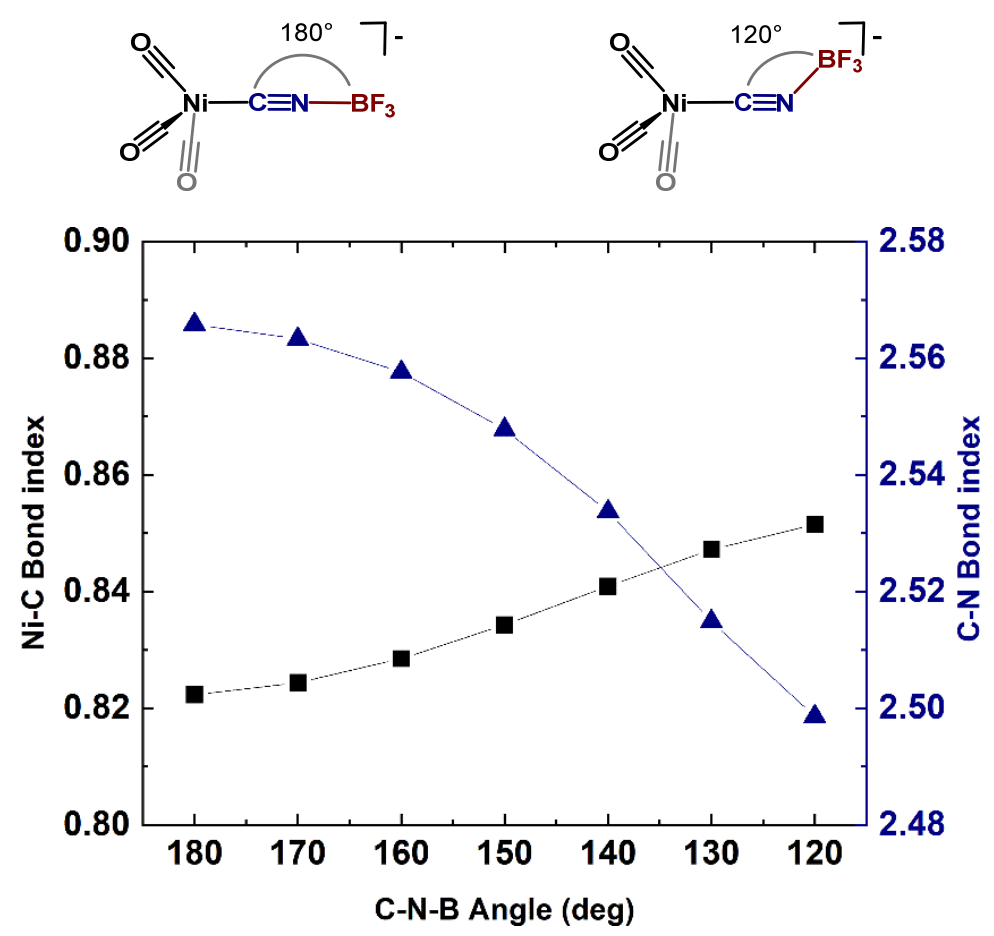

Figure S16. Ni-C (black) and C-N (blue) Wiberg bond indices of $\left[\mathrm{Ni}(\mathrm{CO})_{3}\left(\mathrm{CN}-\mathrm{BF}_{3}\right)\right]^{-}$as a function of $\mathrm{C}-\mathrm{N}-\mathrm{B}$ angle from a relaxed potential energy scan. 

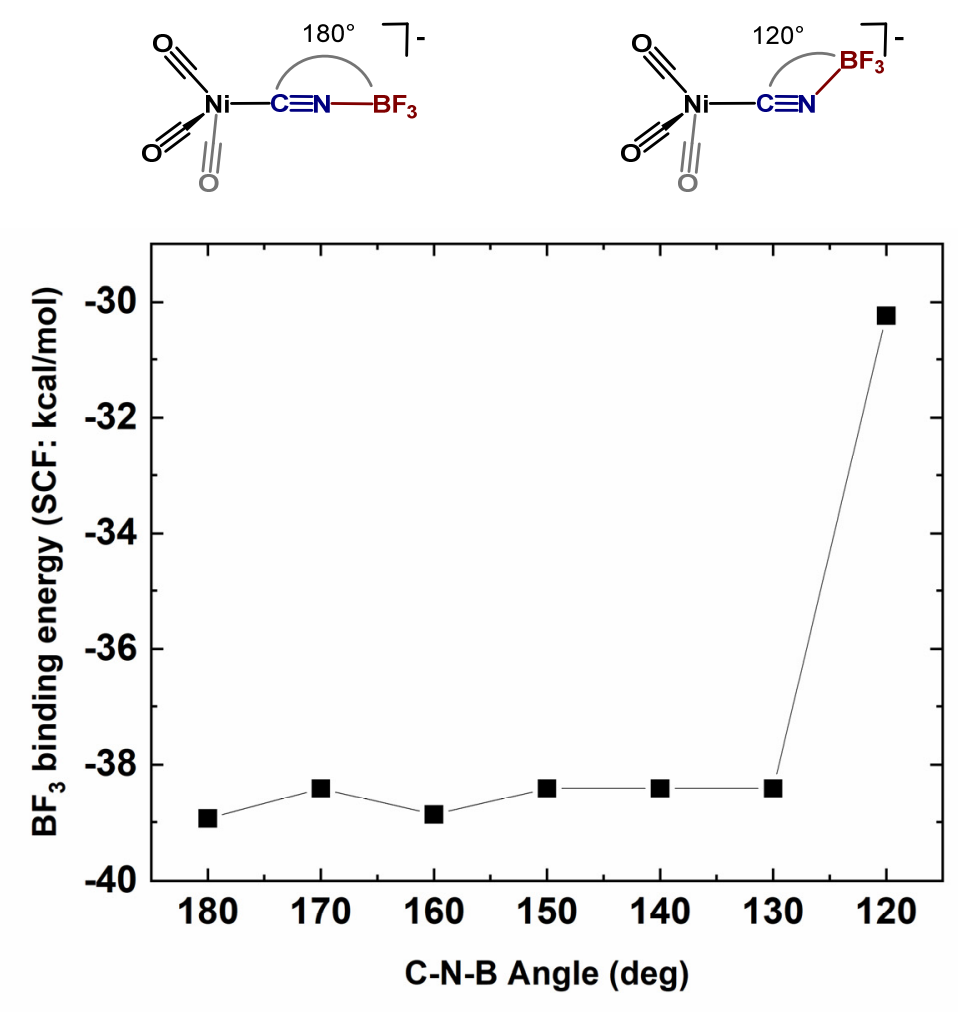

Figure S17. Relative energies $(\mathrm{kcal} / \mathrm{mol})$ of $\left[\mathrm{Ni}(\mathrm{CO})_{3}\left(\mathrm{CN}-\mathrm{BF}_{3}\right)\right]^{-}$as a function of $\mathrm{C}-\mathrm{N}-\mathrm{B}$ angle from a relaxed potential energy scan.

Table S54. Relevant orbital energies $(\mathrm{eV})$ of $\left[\mathrm{Ni}(\mathrm{CO})_{3}(\mathrm{CN})\right]^{-}$, $\left[\mathrm{Ni}(\mathrm{CO})_{3}\left(\mathrm{CN}-\mathrm{BF}_{3}\right)\right]^{-}$, $\left[\mathrm{Ni}(\mathrm{CO})_{3}\left(\mathrm{CN}-\mathrm{BF}_{3}\right)\right]^{-}$with a $120^{\circ} \mathrm{C}-\mathrm{N}-\mathrm{B}$ bend.

\begin{tabular}{|c|c|c|c|}
\hline & {$\left[\mathrm{Ni}(\mathrm{CO})_{3}(\mathrm{CN})\right]^{-}$} & {$\left[\mathrm{Ni}(\mathrm{CO})_{3}\left(\mathrm{CN}-\mathrm{BF}_{3}\right)\right]^{-}$} & {$\left[\mathrm{Ni}(\mathrm{CO})_{3}\left(\mathrm{CN}-\mathrm{BF}_{3}\right)\right]^{-}$(bent) } \\
\hline CN pi* (orth) & 2.571 & 1.253 & 1.265 \\
\hline CN pi* & 2.571 & 1.257 & 0.599 \\
\hline HOMO & -6.008 & -6.539 & -6.475 \\
\hline $\mathrm{Md}+\mathrm{CN}$ pi* (orth) & -6.293 & -6.672 & -6.711 \\
\hline $\mathrm{Md}+\mathrm{CN}$ pi* 2 & -6.293 & -6.680 & -6.808 \\
\hline CN pi (orth) & -8.533 & -9.792 & -9.599 \\
\hline CN pi 2 & -8.534 & -9.796 & -9.735 \\
\hline
\end{tabular}



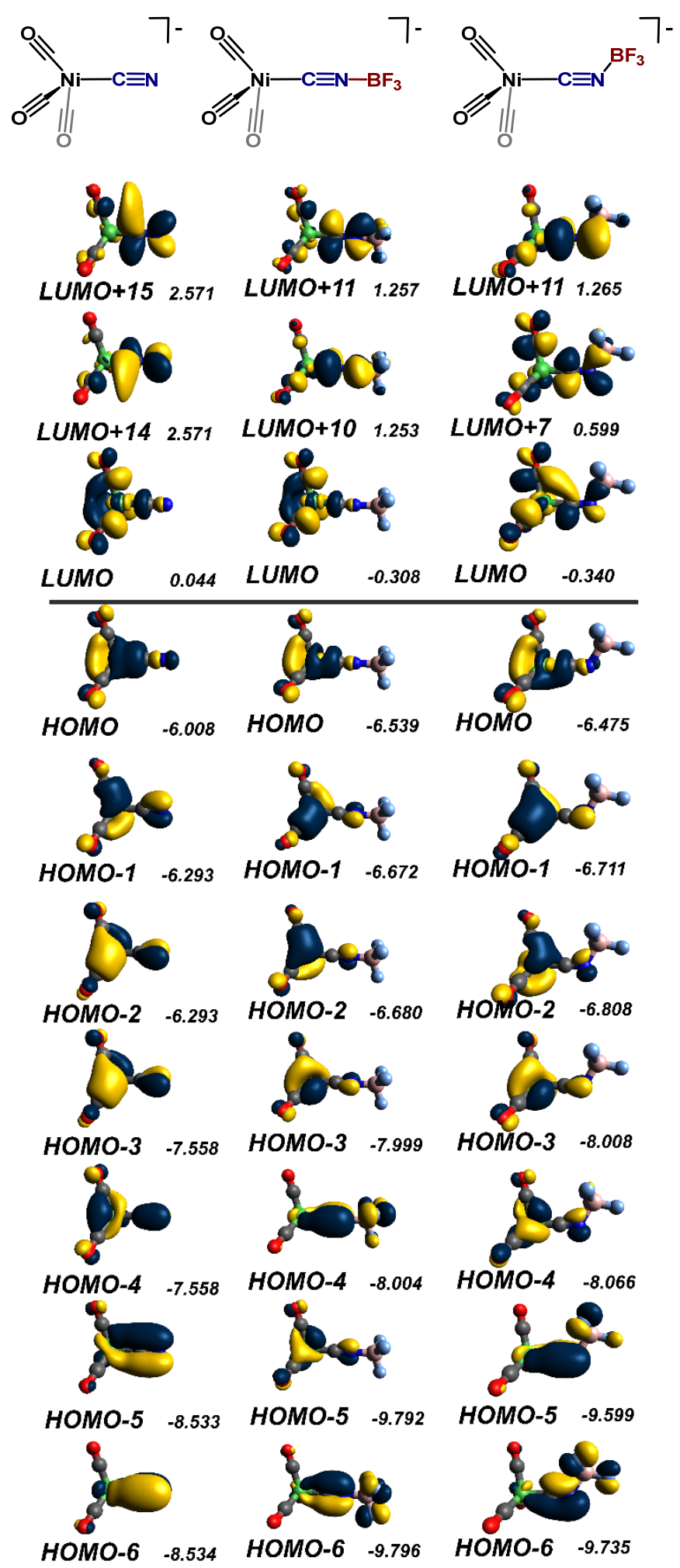

Figure S18. Orbital renderings of representative frontier orbitals of $\left[\mathrm{Ni}(\mathrm{CO})_{3}(\mathrm{CN})\right]^{-}$(left) and $\mathrm{BF}_{3}$ adduct (center) and $\mathrm{BF}_{3}$ adduct at $120^{\circ} \mathrm{CNB}$ angle (right). 


\section{References}

1. Frisch, M. J.; Trucks, G. W.; Schlegel, H. B.; Scuseria, G. E.; Robb, M. A.; Cheeseman, J. R.; Scalmani, G.; Barone, V.; Petersson, G. A.; Nakatsuji, H.; Li, X.; Caricato, M.; Marenich, A. V.; Bloino, J.; Janesko, B. G.; Gomperts, R.; Mennucci, B.; Hratchian, H. P.; Ortiz, J. V.; Izmaylov, A. F.; Sonnenberg, J. L.; Williams; Ding, F.; Lipparini, F.; Egidi, F.; Goings, J.; Peng, B.; Petrone, A.; Henderson, T.; Ranasinghe, D.; Zakrzewski, V. G.; Gao, J.; Rega, N.; Zheng, G.; Liang, W.; Hada, M.; Ehara, M.; Toyota, K.; Fukuda, R.; Hasegawa, J.; Ishida, M.; Nakajima, T.; Honda, Y.; Kitao, O.; Nakai, H.; Vreven, T.; Throssell, K.; Montgomery Jr., J. A.; Peralta, J. E.; Ogliaro, F.; Bearpark, M. J.; Heyd, J. J.; Brothers, E. N.; Kudin, K. N.; Staroverov, V. N.; Keith, T. A.; Kobayashi, R.; Normand, J.; Raghavachari, K.; Rendell, A. P.; Burant, J. C.; Iyengar, S. S.; Tomasi, J.; Cossi, M.; Millam, J. M.; Klene, M.; Adamo, C.; Cammi, R.; Ochterski, J. W.; Martin, R. L.; Morokuma, K.; Farkas, O.; Foresman, J. B.; Fox, D. J. Gaussian 16 Rev. A03-avx2, Wallingford, CT, 2016.

2. Foster, J. P.; Weinhold, F., Natural hybrid orbitals. J. Am. Chem. Soc. 1980, 102 (24), 7211-7218.

3. Gusev, D. G., Electronic and Steric Parameters of $76 \mathrm{~N}$-Heterocyclic Carbenes in $\mathrm{Ni}(\mathrm{CO}) 3(\mathrm{NHC})$. Organometallics 2009, 28 (22), 6458-6461.

4. Gusev, D. G., Donor Properties of a Series of Two-Electron Ligands. Organometallics 2009, 28 (3), 763-770.

5. Adamo, C.; Barone, V., Exchange functionals with improved long-range behavior and adiabatic connection methods without adjustable parameters: The MPW and MPW1PW models. The Journal of Chemical Physics 1998, 108 (2), 664-675.

6. Rassolov, V. A.; Ratner, M. A.; Pople, J. A.; Redfern, P. C.; Curtiss, L. A., 6-31G* basis set for thirdrow atoms. J. Comput. Chem. 2001, 22 (9), 976-984.

7. Ditchfield, R.; Hehre, W. J.; Pople, J. A., Self-Consistent Molecular-Orbital Methods. IX. An Extended Gaussian-Type Basis for Molecular-Orbital Studies of Organic Molecules. The Journal of Chemical Physics 1971, 54 (2), 724-728.

8. Lathem, A. P.; Heiden, Z. M., Quantification of Lewis acid induced Brønsted acidity of protogenic Lewis bases. Dalton Trans. 2017, 46 (18), 5976-5985.

9. Krishnamurthy, V. M.; Semetey, V.; Bracher, P. J.; Shen, N.; Whitesides, G. M., Dependence of Effective Molarity on Linker Length for an Intramolecular Protein-Ligand System. J. Am. Chem. Soc. 2007, 129 (5), 1312-1320.

10. Kiernicki, J. J.; Shanahan, J. P.; Zeller, M.; Szymczak, N. K., Tuning ligand field strength with pendent Lewis acids: access to high spin iron hydrides. Chem. Sci. 2019.

11. Brown, H. C.; Krishnamurthy, S.; Yoon, N. M., Selective reductions. XXI. 9-

Borabicyclo[3.3.1]nonane in tetrahydrofuran as a new selective reducing agent in organic synthesis. Reaction with selected organic compounds containing representative functional groups. The Journal of Organic Chemistry 1976, 41 (10), 1778-1791.

12. Nicasio, J. A.; Steinberg, S.; Ines, B.; Alcarazo, M., Tuning the Lewis Acidity of Boranes in Frustrated Lewis Pair Chemistry: Implications for the Hydrogenation of Electron-Poor Alkenes. Chem.Eur. J. 2013, 19 (33), 11016-11020.

13. Lo, W.; Hu, C.; Berenson, T.; Tracer, N.; Shlian, D.; Khaloo, M.; Benhaim, A.; Jiang, J., Oxidation of carbon monoxide in basic solution catalyzed by nickel cyano carbonyls under ambient conditions and the prototype of a CO-powered alkaline fuel cell. Chem. Commun. 2015, 51 (46), 9432-9435.

14. National Research Council, Acute Exposure Guideline Levels for Selected Airborne Chemicals: Volume 6. The National Academies Press: Washington, DC, 2008; p 320.

15. Barsan, M. E., NIOSH pocket guide to chemical hazards. 2007. 
